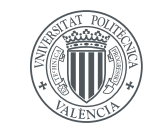

UNIVERSITAT

POLITECCNICA

DE VALÈNCIA

\title{
THE EFFECT OF "POSTURAL FREEDOM" IN LAPAROSCOPIC SURGERY
}

\section{Horacio M. Pace Bedetti}

Directores:

Dr. Andrés Conejero \& Dr. José Luis Martínez de Juan

Valencia, Abril, 2019 



\section{a mi familia}





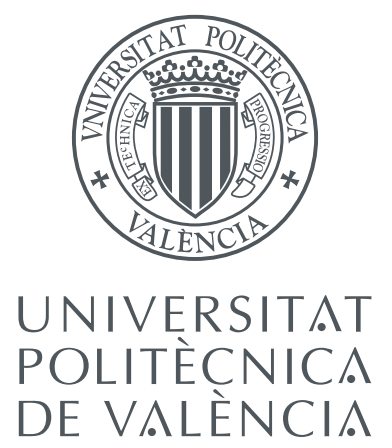

\title{
THE EFFECT OF “POSTURAL FREEDOM" IN LAPAROSCOPIC SURGERY
}

\author{
Thesis presented by \\ Horacio M. Pace Bedetti
}

to obtain the degree of

$\mathrm{PhD}$ in Technologies for Health and Well-Being

Universitat Politècnica de València, 2019

Directores:

Dr. Andrés Conejero

Dr. José Luis Martínez de Juan 

Index 
Abstract

Resumen

Resum

- Table of Content

Figures

Tables

Abbreviations

- Chapter 1. Introduction

1. Abdomen and conventional open surgery

1.1. Abdomen

1.2. Laparotomy

2. Laparoscopic surgery

2.1. Procedure and instrumentation

2.1.1. Laparoscopic conventional hand-held instruments

2.1.2. The pistol grip handle and the instrument tips

2.2. Advantages of laparoscopy

2.3. Disadvantages of laparoscopy

3. Fatigue and musculoskeletal disorders associated to laparoscopic surgery

3.1. Fatigue

3.2. Fatigue associated to laparoscopic surgery

3.3. Musculoskeletal disorders

3.4. Ergonomic and musculoskeletal disorders associated to laparoscopic surgery

4. The design of ergonomic laparoscopic hand-held instruments

4.1. Requirements for an ergonomic laparoscopic handle

4.2. Solutions presented by other research teams 
5. Justification

5.1.. Key points that justify this manuscript

5.2.. The concept of "Postural Freedom"

6. Objectives

65

6.1. General objectives

6.2. Specific objectives

- Chapter 3. Electromyography evaluation

7. Introduction. EMG in ergonomic studies

7.1. Skeletal muscle anatomy

7.1.1. Micro anatomy

7.1.2. Macro anatomy. Muscles affected during laparoscopic procedures.

7.2. Muscle performance

7.2.1. Physiology

7.2.2. Electrical signal associated to the skeletal muscle

7.3. $\quad$ EMG recording techniques

7.3.1. Intramuscular EMG

7.3.2. Surface EMG

7.4. Surface EMG analysis

7.4.1. Analysis to measure the muscular effort. Amplitude parameter

7.4.2. Analysis to measure the muscular fatigue. Mean and median frequency parameter

7.5. Muscular fatigue index

7.5.1. Failure point approach

7.5.2. Spectral modifications approach

7.5.3. JASA approach

7.6. Other methods to detect muscular fatigue

7.7. Introduction to the experiments

7.8. Refining the experiment 
8. Materials and methods

8.1. Participants

8.2. Instruments

8.3. Protocol

8.4. Data acquisition

8.5. Data analysis

9. Results 101

10. Discussion 108

- Chapter 4. Motion capture analysis

11. Introduction. Motion capture in ergonomic studies

11.1. Critical movements in laparoscopic surgery

11.1.1. The VBST system

11.1.1.1. Marker-based

11.1.1.2. Depth-based

11.1.1.3. Contrast-based

11.2. Introduction to the experiment

11.3. Looking the appropriate MoCap system

12. Materials and methods

12.1. Participants

12.2. Instruments

12.3. Protocol

12.4. Data acquisition

12.5. Data analysis

13. Results

135

$14 . \quad$ Discussion

- Chapter 5. Design and prototype of a PF instrument for laparoscopic surgery. User-centered design

15. Introduction. The user-centered design

15.1. User-centered methodology 
16. Creating the design solution. Evotool

16.1. Design of a PF hand-held laparoscopic instrument

16.2. Prototyping

16.3. Evotool

17. Evaluating the solution with real users

17.1. User-based evaluations

17.2. Test with expert users

17.3. Test Results

17.4. Discussion

- Chapter 6. Conclusions 

Abstracts 


\section{Abstract}

Laparoscopic surgery is considered one of the main surgical advances in the last decades, this technique has demonstrated numerous advantages compared to open conventional surgery and it is widely used in abdominal procedures around the world. For the patient, laparoscopic surgery suppose less post-operative pain, shorter recovery time, lower risk of infection, and reduction of the trauma among other benefits.

For the surgeon, the situation is completely different, this practice requires more effort, concentration and mental stress than conventional open procedures. It forces the surgeon to adopt non-neutral postures with phalanges, hands, wrists, and arms being this non-neutral postures the main cause of muscular fatigue and high risk of musculoskeletal disorders. The poor ergonomic postures accelerate muscle fatigue and pain because, outside the neutral range, muscles require more energy to generate the same contractile force than in neutral position. This increase of muscular fatigue is associated with the potential to commit errors that may harm the patient during the surgery.

Because this problem is widely studied and different research centers are already trying to improve their surgeons experience in the operation room, the approach used during this work is different than most of the ones presented in previous works. Generally, the solutions proposed are based on ergonomic changes in the handle shape of the instrument, because the conventional pistol-grip handle is considered ergonomically poor. But the problem is not only in the shape of the handle but also in the fixed point of entrance that force the positions for the surgeon despite the handle's shape.

In this work, the concept of postural freedom in laparoscopic surgery is introduced and evaluated. The postural freedom concept is based on the hypothesis that the surgeon involuntarily would maintain neutral postures if the instrument does not force him or her to reach extreme position with the upper limbs. 
The benefits of this concept has been demonstrated, by means of electromyography and motion capture. It reduces the localized muscular fatigue and increases the number of neutral postures during laparoscopic simulations.

In the final section it is proposed a design that implements the postural freedom concept with, according on the results, the potential to reduce the localized muscular fatigue and the musculoskeletal problems associated to the practice.

The design proposed here acts as a new section on the arm, being an articulation that support the turns and big displacements that currently suffer the surgeon's body. The solution is affordable and easy to manufacture and could be used by surgeons worldwide. 


\section{Resumen}

La cirugía laparoscopia está considerada uno de los principales avances quirúrgicos en las últimas décadas. Esta técnica ha demostrado numerosas ventajas comparadas con la cirugía convencional abierta y ha sido extensamente usada para procesos quirúrgicos en el área abdominal. Para el paciente, la cirugía laparoscópica supone diversas ventajas, como por ejemplo menor dolor post operativo, tiempos de recuperación menores, menor riesgo de infección, o reducción del trauma.

Para el cirujano en cambio, la situación es completamente diferente, esta práctica requiere mayor esfuerzo, concentración y estrés mental que la práctica convencional abierta. Además fuerza al cirujano a adoptar posiciones no-neutras en falanges, manos, muñecas, y brazos. Estas posturas no-neutras son la principal causa de fatiga muscular y aumentan el riesgo de problemas musculo-esqueléticos.

Estos problemas han sido ampliamente estudiados por diferentes equipos de investigación, los cuales están tratando de mejorar la experiencia del cirujano en el quirófano. El enfoque utilizado en este estudio es diferente del utilizado anteriormente por la mayoría de estos equipos, los cuales suelen propones soluciones basadas en cambios ergonómicos con la intención de mejorar la geometría del mango de pistola convencional, ya que se considera ergonómicamente deficiente. El problema con este enfoque, es que las deficiencias no se encuentran únicamente en el mango, sino en la utilización de un punto de entrada fijo que fuerza a los cirujanos a mantener posiciones desfavorables.

En este trabajo, se introduce el concepto "Libertad Postural" en el ámbito de la cirugía, este se basa en la hipótesis de que, si las herramientas no forzaran la posición de los cirujanos, estos mantendrían posiciones más favorables y cercanas al rango de posiciones neutras durante los procesos laparoscópicos. 
Los beneficios de este concepto han sido demostrados por medio de análisis de movimiento y de electromiografía de superficie, los cuales indican que la "Libertad Postural" es causante de un claro aumento de las posiciones neutras y de la reducción de la fatiga muscular, y han sido testeados por cirujanos en entornos simulados, los cuales encuentran beneficioso utilizar la "Libertad Postural" como característica base de este nuevo diseño de herramienta laparoscópica.

En la sección final de este trabajo se propone un diseño que implementa el concepto de libertad postura con el cual se reduciría la fatiga muscular y los problemas musculo esqueléticos asociados a la práctica laparoscópica.

Este diseño tiene la característica de actuar como una nueva sección del brazo, siendo una articulación que soporta los giros y grandes desplazamientos que normalmente tienen que desarrollar los brazos del cirujano. Además, esta solución es económica y fácil de fabricar, lo cual permitiría su uso por cirujanos de todo el mundo. 


\section{Resum}

La cirurgia laparoscòpia està considerada un dels principals avanços quirúrgics en les últimes dècades. Aquesta tècnica ha demostrat nombrosos avantatges comparats amb la cirurgia convencional oberta i ha sigut extensament usada per a processos quirúrgics en l'àrea abdominal. Per al pacient, la cirurgia laparoscòpica suposa diversos avantatges, com per exemple menor dolor post operatiu, temps de recuperació menors, menor risc d’infecció, o reducció del trauma.

Per al cirurgià en canvi, la situació és completament diferent, aquesta pràctica requereix major esforç, concentració i estrés mental que la pràctica convencional oberta. A més força al cirurgià a adoptar posicions no-neutres en falanges, mans, nines, i braços. Aquestes postures no-neutres són la principal causa de fatiga muscular i augmenten el risc de problemes musculo-esquelètics.

Aquests problemes han sigut àmpliament estudiats per diferents equips d'investigació, els quals estan tractant de millorar l'experiència del cirurgià en el quiròfan. L'enfocament utilitzat en aquest estudi és diferent de l'utilitzat anteriorment per la majoria d'aquests equips, els quals solen proposes solucions basades en canvis ergonòmics amb la intenció de millorar la geometria del mànec de pistola convencional, ja que es considera ergonòmicament deficient. El problema amb aquest enfocament, és que les deficiències no es troben únicament en el mànec, sinó en la utilització d'un punt d'entrada fix que força als cirurgians a mantindre posicions desfavorables.

En aquest treball, s'introdueix el concepte "Llibertat Postural" en l'àmbit de la cirurgia, aquest es basa en la hipòtesi que, si les eines no forçaren la posició dels cirurgians, aquests mantindrien posicions més favorables i pròximes al rang de posicions neutres durant els processos laparoscòpics. 
Els beneficis d'aquest concepte han sigut demostrats per mitjà d'anàlisi de moviment i de electromiografía de superfície, els quals indiquen que la "Llibertat Postural" és causant d'un clar augment de les posicions neutres i de la reducció de la fatiga muscular, i han sigut testats per cirurgians en entorns simulats, els quals troben beneficiós utilitzar la "Llibertat Postural" com a característica base d'aquest nou disseny d'eina laparoscòpica.

En la secció final d'aquest treball es proposa un disseny que implementa el concepte de llibertat postura amb el qual es reduiria la fatiga muscular i els problemes *musculo esquelètics associats a la pràctica laparoscòpica.

Aquest disseny té la característica d'actuar com una nova secció del braç, sent una articulació que suporta els girs i grans desplaçaments que normalment han de desenvolupar els braços del cirurgià. A més, aquesta solució és econòmica i fàcil de fabricar, la qual cosa permetria el seu ús per cirurgians de tot el món. 

Table of content 


\section{Figures}

- Figure 1. Abdominal cavity. Female pelvic viscera and abdominal wall. Adapted from the drawings created by Corbyn Beach in 2009 for the biomedical communications program at the UT Southwestern Graduate School of Biomedical Sciences.

- Figure 2. Laparoscope (Hipp Endoskop Service, Freiburg, Germany). The camera is attached to the external part (black element) of the laparoscope.

- Figure 3. The Nitze's cystoscope. Figure adopted from the European Museum of Urology.

- Figure 4. Veress needle (Stryker, MI, USA).

- Figure 5. Hasson's blunt-tipped trocar (Genicon, Florida, USA). The Hasson's technique is one of the most commonly used ways to perform the open entry technique.

- Figure 6. Trocar. Versaport Plus Bladeless Trocar (Medtronic, Minneapolis, USA).

- $\quad$ Figure 7. Needle driver (Matrix Surgical, Georgia, USA).

- Figure 8. Laparoscopic grasper (Teleflex, North Carolina, USA).

- Figure 9. Conventional configuration of a pistol grip handle (Surgical Innovation, Leeds, UK).

- Figure 10. Snowden-Pencer (FarmPD, New Hampshire, USA).

- Figure 11. Single action jaw (left) and double action jaw (right) grasping forceps by BD (New Jersey, USA).

- Figure 12. Different double jaw graspers on the market. Adopted from a document of the World Laparoscopic Hospital [15].

- Figure 13. a) Double action curved scissor, b) Single action curved microscissors by BD, c) Single action hooked scissors by BD, and d) Straight double action blade without serration by BD (New Jersey, USA).

- Figure 14. Surgeons posture during laparoscopic procedure.

- Figure 15. Most important functional zones of the palm for manipulating laparoscopic handles. Adapted from [64]. 
- Figure 16. Prototype ball handle needle driver. A) Line grip and B) transverse grip. Figure adapted from [32].

- Figure 17. Design of the Rocker handle needle driver. Figure adapted from [67].

- Figure 18. Prototype Intuitool. Developed by the University of Nebraska.

- Figure 19. FlexDex instrument (FlexDex Surgical, Michigan, USA).

- Figure 20. Maestro instrument. Figure adapted from [73].

- Figure 21. Muscle architecture presented by R. Lieber in 2009. Adapted from [79].

- Figure 22.Schematic illustration of different types of muscles: (a) fusiform, (b) unipennate, and (c) bipennate. Adapted from [77].

- Figure 23. Diagrammatic explanation of the spectral modification that occurs in the EMG signal during sustained contractions. The muscle fatigue index is represented by the median frequency of the spectrum. Adapted from [101].

- Figure 24. Myoelectric signal amplitude and force during an attempted constant-force contraction in the first dorsal inter-osseous muscle. Adapted from [22].

- Figure 25. EMG signal and PSD obtained from one of the experiments with the conventional instrument.

- Figure 26. Contractile fatigue. The failure point denotes the time when the force output was no longer maintained at the desired average value. The time duration of the contraction was 150 seconds. Adapted from [22].

- Figure 27. Distinction between contractile fatigue and metabolic fatigue. The metabolic fatigue is observed at the beginning of the contraction while the failure point appears once the contraction cannot be maintained anymore. The time duration of the contraction was 150 seconds. Adapted from [22].

- Figure 28. JASA scenarios.

- Figure 29. Trapezius muscle. Blue color identify the superior. Figure obtained from Visible Body Software.

- Figure 30. Deltoids muscle. Blue color identify the anterior fibers. Figure obtained from Visible Body Software.

- Figure 31. Biceps muscle. Blue color identify whole muscle. Figure obtained from Visible Body Software. 
- Figure 32. Flexor carpi radials. Blue color identify the whole muscle. Figure obtained from Visible Body Software

- Figure 33. Curve test adapted from Mattern et al. [144]. The red dots were included to record some extreme positions, arrows indicate the direction of the test.

- Figure 34. Raw signal obtained on the dynamic experiment with the control instrument. The signals belongs to muscles trapezius, deltoids, biceps, and flexor carpi radials in descending order. Values in millivolts $(\mathrm{mV})$.

- Figure 35. Raw signals of 2 minutes of the dynamic experiment with the control instrument. Red circles identify patterns founded in the signal. The signals belongs to muscles trapezius, deltoids, biceps, and flexor carpi radials in descending order. Values in millivolts $(\mathrm{mV})$.

- Figure 36. Raw signal generated by the dynamic experiment with both instrument. Left signals correspond to the control instrument and right signal to the prototype instrument. The signals belongs to muscles trapezius, deltoids, biceps, and flexor carpi radials in descending order. Values in millivolts $(\mathrm{mV})$.

- Figure 37. Left picture indicate the participant location in front of the box trainer simulator. The shoulder is aligned with the target $0^{\circ}$. The trocar entrance is located above of the center of the template. The right picture is the $360^{\circ}$ template used during the experiment. The participant holds the tip of the instrument at each target for 55 seconds.

- Figure 38. Raw signal of one participant during the experiment with control instrument. Each minute represents a target. Initial and final minute are rest positions. The muscles a) trapezius, b) deltoids, c) biceps and d) flexor carpi radials are presented.

- Figure 39. Both instrument used to compare the effectiveness of PF in hand-held instruments. Superior instrument is a conventional pistol-grip handle. Inferior instrument is the PF prototype.

- Figure 40. Box-trainer and trocar used in the sEMG experiment.

- Figure 41. 360 Degrees template. During the experiment, this template is located centered below of the trocar incision to force the subject to reach positions with different level of effort during the experiment.

- Figure 42. A schematic of the differential amplifier configuration. The EMG signal is represented by " $m$ " and the noise signals by " $n$ ". Adapted 
from [147].

- Figure 43. RMS $(\% \pm \mathrm{SE})$ values of trapezius muscle in each target. The values are normalized respect the Entrance target with the control instrument. Blue bars represent the control instrument values and orange bars the PF prototype values.

- Figure 44. RMS $(\% \pm \mathrm{SE})$ values of deltoids muscle in each target. The values are normalized respect the Entrance target with the control instrument. Blue bars represent the control instrument values and orange bars the PF prototype values.

- Figure 45. RMS $(\% \pm \mathrm{SE})$ values of biceps muscle in each target. The values are normalized respect the Entrance target with the control instrument. Blue bars represent the control instrument values and orange bars the PF prototype values.

- Figure 46. RMS $(\% \pm \mathrm{SE})$ values of flexor carpi radials muscle in each target. The values are normalized respect the Entrance target with the control instrument. Blue bars represent the control instrument values and orange bars the PF prototype values. Notice the change of scale.

- Figure 47. Trapezius RMS values by participant during the whole experiment. Horizontal axis indicate the number of the participant. Vertical axis the value in millivolts $(\mathrm{mV})$. Prototype orange bars. Control blue bars.

- Figure 48. Deltoids RMS values by participant during the whole experiment. Horizontal axis indicate the number of the participant. Vertical axis the value in millivolts $(\mathrm{mV})$. Prototype orange bars. Control blue bars.

- Figure 49. Biceps RMS values by participant during the whole experiment. Horizontal axis indicate the number of the participant. Vertical axis the value in millivolts $(\mathrm{mV})$. Prototype orange bars. Control blue bars.

- Figure 50. Flexor Carpi Radials RMS values by participant during the whole experiment. Horizontal axis indicate the number of the participant. Vertical axis the value in millivolts $(\mathrm{mV})$. Prototype orange bars. Control blue bars.

- Figure 51. Result of Fmed and RMS and the interpretation by JASA of the results presented by all the participants together. Upward arrow indicates an increase between entrance and exit targets. Downward arrow indicates a decrease between entrance and exit targets.

- Figure 52. Ergonomically ideal position for the laparoscopic surgeon. Adapted from [64]. 
- Figure 53. Static position during surgery. Adapted from [39].

- Figure 54. Basic VICON setup to evaluate an archer motions. Acquired from Engadget magazine.

- Figure 55. Kinect interference problem. The column C1 shows the depth images of each sensor with no interference. In the column C2 the depth images of each camera in a system composed by two cameras reflect the interference problem. The column $\mathrm{C} 3$ reflect the missed information caused by the interference. Adapted from [171].

- Figure 56. Tracker Video Analysis Software. In this image, the software is tracking the position and time of the ball. Red rhombus in the picture mark the position of the ball on each frame.

- Figure 57. Angular movements: abduction, adduction, and circumduction of the upper limb at the shoulder. Adapted from [176].

- Figure 58. Angular movements: flexion and extension at the shoulder and knees. Adapted from [176].

- Figure 59. Ranges of arm flexion-extension, forearm flexion, and arm abduction-adduction according by REBA and RULA methods.

- Figure 60. Suture pad used during the experiment at IMSaT laboratory (Dundee, Scotland).

- Figure 61. Laparoscopic surgeon being recorded by VICON system. Picture of the study performed at IMSaT laboratory in the Ninewells Hospital (Dundee, Scotland) during my internship. Red circles mark only a fraction of the cameras used during this experiment.

- Figure 62.Testing the setup composed by two Kinect V2 sensors at IMSaT (Dundee, Scotland).

- Figure 63. Setup for the tests performed by two Kinect V2. Adapted from [179].

- Figure 64. Positions to track with Tracker Vision System. The red point indicates the shoulder, the blue point indicates the elbow and the yellow point indicates the wrist. Screenshot from the test performed in the Area of Clinical Simulation (Hospital la Fe).

- Figure 65. Point to follow and region to search in shoulder. Screenshot from the test with a participant in the study developed in the Area of Clinical Simulation (Hospital la Fe).

- Figure 66. Tracker Vision System tracking the trajectory of the wrist. Axis point (purple) located in the elbow. Screenshot from the test with a participant in the study developed in the Area of Clinical Simulation 
(Hospital la Fe).

- Figure 67. PF prototype used during the MoCap experiment. The handle has a cylindrical shape in order to avoid the pain caused by the rings of the conventional pistol-grip handle.

- Figure 68. Control instrument used during the experiments.

- Figure 69. Box trainer model Szabo-Berci-Sackier (Karl Storz, Germany) used in the area of clinical simulation of the Hospital La Fe (Valencia, Spain).

- Figure 70. Curve test. The shape of the path was adapted from Matern et al. [144]. Red dots included to record some extreme positions, arrows indicate the direction of the test.

- Figure 71. Tracking of elbow and wrist with the Tracker Video Analysis software.

- Figure 72. Arm abduction values by instrument. Boxes indicate the main amount of values (percentiles 25 to 75 ). The error bars indicate the maximum and minimum value reached.

- Figure 73. Arm adduction values by instrument. Boxes indicate the main amount of values (percentiles 25 to 75 ). The error bars indicate the maximum and minimum value reached.

- Figure 74. Arm flexion values by instrument. Boxes indicate the main amount of values (percentiles 25 to 75 ). The error bars indicate the maximum and minimum value reached.

- Figure 75. Arm extension values by instrument. Boxes indicate the main amount of values (percentiles 25 to 75 ). The error bars indicate the maximum and minimum value reached.

- Figure 76. Forearm flexion values by instrument. Boxes indicate the main amount of values (percentiles 25 to 75). The error bars indicate the maximum and minimum value reached.

- Figure 77. Laparoscopic surgeon performing an intervention. In the picture is easily recognizable the excessive arm abduction and wrist flexion to perform the procedure.

- Figure 78. Some of the low fidelity prototypes in different steps of the process developed during this thesis.

- Figure 79. Low fidelity prototype evaluation in Hospital La Fe of Valencia (Spain). 
- Figure 80. Sagittal plane section, $45^{\circ}$ view. PF prototype (Evotool).

- Figure 81. Sagittal and transversal planes sections, $0^{\circ}$ and $45^{\circ}$ view. PF prototype (Evotool).

- Figure 82. Comparison for two positions with the Evotool prototype and a conventional instrument. In the figure a) the conventional instrument reach the target 270 and figure $b$ ) the PF prototype reaching the same target. In figure c) a different perspective with the conventional instrument reaching the target 90 and figure d) the PF prototype reaching the same target.

- Figure 83. Evotool rotation steps. With Evotool, a) if the surgeon needs to change the angle of the distal tip, is juts necessary $b$ ) to slightly turn the wrist in order to reach the appropriate angle, once is reached c) the surgeon can recover a position more comfortable for the wrist.

- Figure 84. Prototype used with the final users. It was a functional prototype in an early stage. The users can interact with it and use it to perform simple tasks.

- Figure 85. Pick and place test performed in the Hospital la Fe. The video was obtained from the laparoscope record.

- Figure 86. Surgeons performing the pick and place test before the survey to test the usability of the PF prototype.

- Figure 87. The two handle design alternatives used during the second part of the experiment. Model 1 left side and Model 2 right side.

\section{Tables}

- Table 1. General and proposed ergonomic requirements for hand tools and dissection forceps. Adapted from [65].

- Table 2. Fmed values (Average \pm SD) of PF prototype and control instrument in Entrance and Exit target, and difference between these values. ${ }^{*}$ Significant differences $(\rho<0,05)$.

- Table 3. JASA evaluation by participant. Results indicate muscular fatigue $(\mathrm{MF})$, force increase (FI), force decrease (FD), and muscle recovery (MR). $(*)=\rho<0,05$. 
- Table 4. RMS error average (SD). First column compare the Kinect results with the real location of the cube. Second column compare the results with the VICON system results.

- Table 5. Percentage of neutral position by each movement with both instruments.

- Table 6. Mean, maximum and difference of the movements performed with each instrument by experts and non-experts.

- Table 7. Survey answers by the surgeons and residents of the Hospital la Fe.

\section{Abbreviations}

- MAS: Minimal Access Surgery

- MIS: Minimal Invasive Surgery

- CNS: Central Nervous System

- EMG: Electromyography

- LMM: Lumbar Motion Monitor

- DoF: Degrees of Freedom

- MoCap: Motion Capture

- OWAS: Ovako Working Posture Analyzing System

- RULA: Rapid Upper Limb Assessment

- NIOSH: National Institute of Occupational Safety and Health

- REBA: Rapid Entire Body Assessment

- SAGES: Society of American Gastrointestinal Endoscopic Surgeons

- sEMG: Surface Electromyography

- MU: Motor Unit

- EA: Electrical Activity

- ARV: Average Rectified Value

- RMS: Root Mean Square

- PSD: Power Spectral Density

- Fm: Mean Frequency 
- Fmed: Median Frequency

- JASA: Joint Analysis of the Spectral and Amplitudes

- MMG: Mechanomyography

- NIRS: Near-Infrared Spectroscopy

- SMG: Sonomyography

- SENIAM: Surface EMG for Non-Invasive Assessment of Muscles

- NVBST: Non Vision-Based Sensor Technology

- VBST: Vision-Based Sensor Technology

- VICON: Variable Independent Control

- IMSaT: Institute for Medical Science and Technologies

- CPOE: Computerized Physician Order Entry 


Chapter 1.
Introduction 
This manuscript deal with the ergonomic problems caused by hand-held instrumentation in laparoscopic surgery. For this reason, the introduction includes the necessary information to understand the abdominal cavity and the surgical techniques performed in this area, but also it tries to improve the ergonomic of surgeons in the operating room and concept as localized muscular fatigue, muscular displacement and usability are also included in posterior sections of the manuscript.

In order to understand laparoscopic technique and the improvement that this technique supposed for surgical procedures, is necessary to start with the abdomen and the conventional surgical procedures for this part of the body.

\section{Abdomen and conventional open surgery \\ 1.1. Abdomen}

The abdomen (Figure 1) is the lower part of the trunk. Above it, and separated from it by the diaphragm, lies the thorax or chest, and below lies the pelvis. The contained organs are protected by the spinal column, lower ribs, iliac bones and down-sloping ribs at the back and the sides. In front, the abdominal content is protected only by soft tissues: skin tissue layer, a varying amount of fat, three layers of broad, flat muscle, another layer of fat, and finally the smooth, thin peritoneum which lines the whole cavity [1].

The principal contents of the abdominal cavity are the digestive organs as stomach, intestine, liver, pancreas, etc. The open procedure used to explore or repair any injury in this part of the body is called laparotomy. 


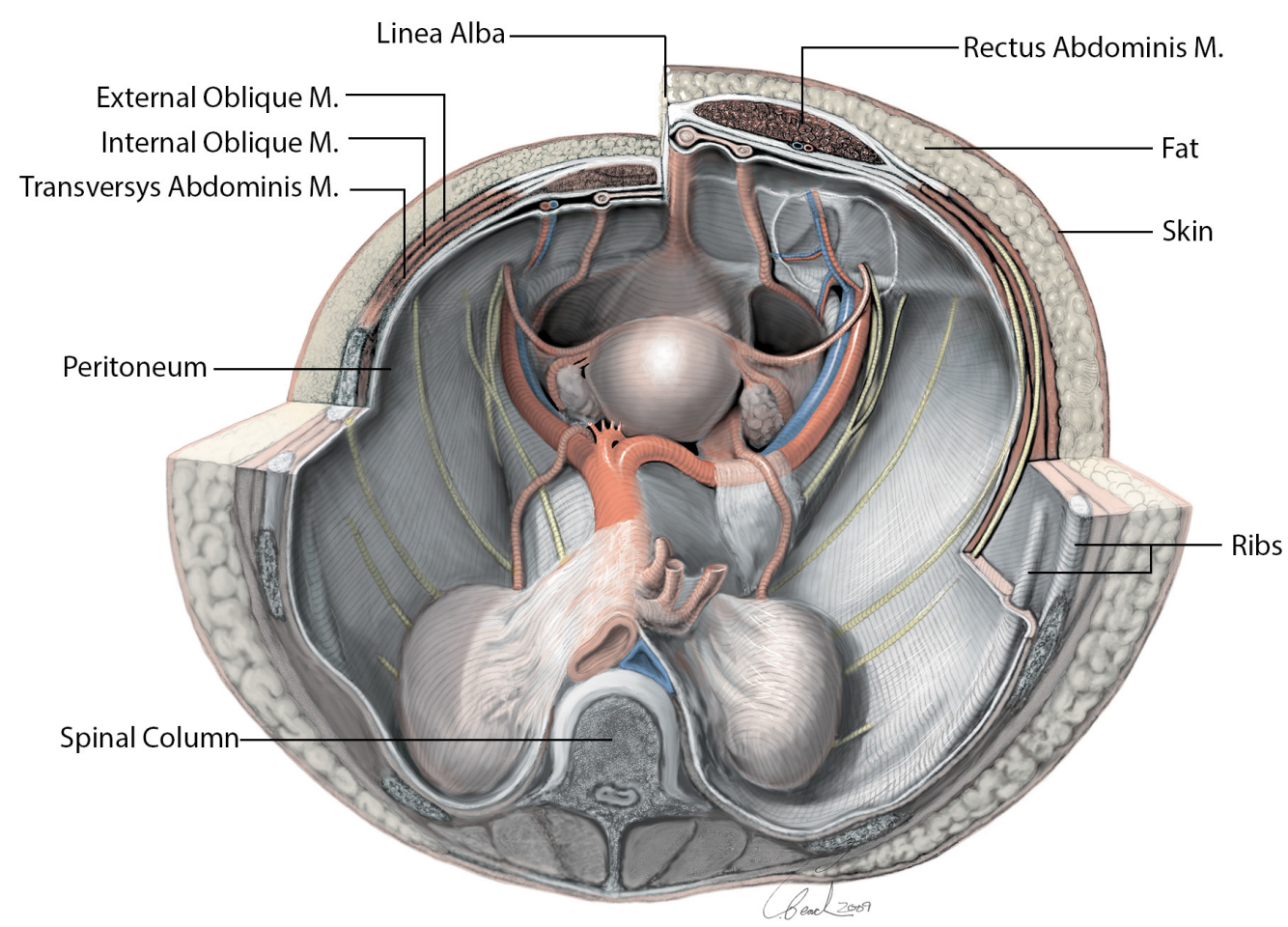

Figure 1. Abdominal cavity. Female pelvic viscera and abdominal wall. Adapted from the drawings created by Corbyn Beach in 2009 for the biomedical communications program at the UT Southwestern Graduate School of Biomedical Sciences.

\subsection{Laparotomy}

The conventional open surgery in the abdominal cavity is called laparotomy. Laparotomy is a surgical procedure performed by means of a large incision that allows surgeons to directly explore the abdominal cavity to inspect the organs and diagnose any problem. This procedure may be recommended for a patient who has abdominal pain of unknown origin or who has suffered an injury in the abdominal area.

Exploratory laparotomy plays an important role in the staging of certain cancers because it allows surgeons to examine directly the abdominal organs for evidence of cancer. 
During the exploration, a range of medical issues may be discovered, such as peritonitis (inflammation of the peritoneum), appendicitis (inflammation of the appendix), pancreatitis (inflammation of the pancreas), abscesses (an infection in a localized area), adhesions (bands of scar tissue that form after trauma or surgery), diverticulitis (inflammation of the pouches formed in the walls of the intestines), intestinal perforation, ectopic pregnancy (fertilized egg attaches outside the uterus of the mother), foreign bodies (gunshot wound) or internal bleeding (road traffic accident).

Due to the large incisions required in laparotomy, the risk of blood loss, infections and operation pain is considered high [2]-[5] but, for surgeons the open procedure has some benefits. In open surgery, surgeons can look at and touch the patient's organs directly with their hands [6], being the exposure of the operating field to direct viewing and the quality and intensity of the light source the main concerns during the procedure [6], [7]. This direct contact is used to easily identify areas with some kind of abnormality [8].

\section{Laparoscopic surgery}

Laparoscopic surgery is a procedure included in the family of minimal access surgeries (MAS), also known as minimal invasive surgery (MIS).

During this procedure, the surgeon examines and manipulates the interior of the abdominal cavity from the exterior through small incisions in the abdominal wall. The surgeon can visualize the interior of the abdominal cavity by means of a rigid endoscope called laparoscope (Figure 2).

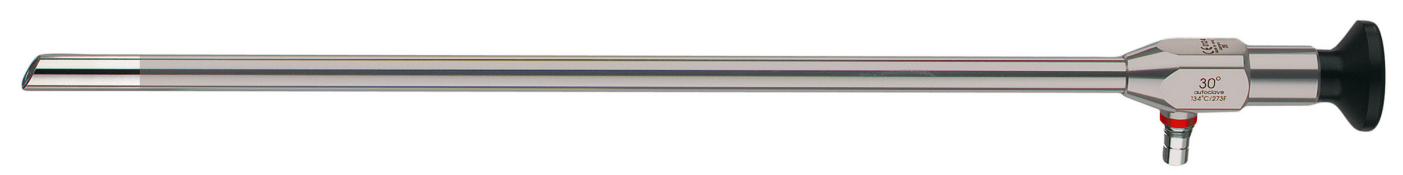

Figure 2. Laparoscope (Hipp Endoskop Service, Freiburg, Germany). The camera is attached to the external part (black element) of the laparoscope. 
The first successful laparoscopy was performed in 1901 [4] by Georg Kelling, a surgeon from Dresden (Germany), who insufflated the abdominal cavity of a dog, inserted a larger trocar and introduced a Nitze's cystoscope (Figure 3) to inspect the abdominal visceral organs. The Nitze's cystoscope is a hollow tube commonly used in endoscopies of the urinary bladder to visualize the intra-corporeal cavity.

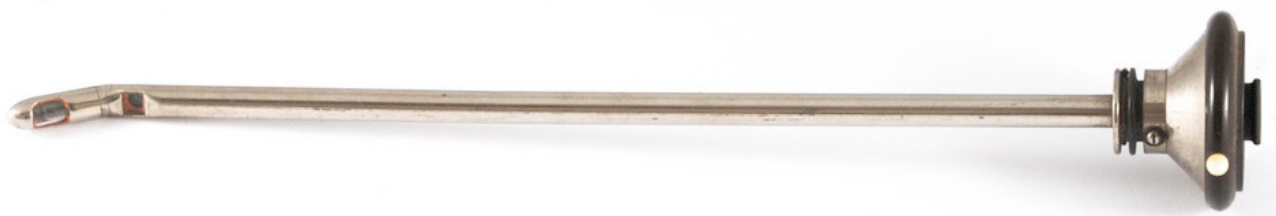

Figure 3. The Nitze's cystoscope. Figure adopted from the European Museum of Urology.

Kelling's achievement was a success, and some years later, in 1910 Hans Christian Jacobaeus of Sweden reported 17 cases in which laparoscopy was accomplished in humans, using a Nitze's cystoscope with "cold burning" lamps and a cannula with a valve system [9]. In 1911 Jacobaeus described 80 laparoscopies with only one reported complication, a hemorrhage into the peritoneal cavity during the trocar incision [10].

After these initial procedures, the laparoscopic surgery has greatly evolved over the years. Now, every single urological operation, oncological or otherwise, can be performed by means of laparoscopy [4]. These procedures are applied in general surgery practices, they keep basic principles of conventional open surgery, and make it possible to access the intra-abdominal cavity and even develop therapeutic procedures.

\subsection{Procedure and instrumentation}

Two approaches are usually used in laparoscopic surgery to enter into the peritoneal cavity [11]:

- The classic closed technique using a Veress needle (Figure 4) to insufflate carbon dioxide gas $\left(\mathrm{CO}_{2}\right)$ before the trocar incision.

- The open technique which uses a blunt tipped trocar, to make this primary incision in a controlled manner. 
In the closed access technique, the pneumoperitoneum (abnormal presence of gas in the peritoneal cavity) is created using a Veress needle, which is a needle equipped with a spring-loaded obturator that is used to insufflate $\mathrm{CO}_{2}$ in the abdomen before the primary trocar incision.

The Veress needle was developed by Dr. Veres (Hungary) in 1933 [12] and is the most commonly practiced way of access by surgeons and gynecologists worldwide [11].

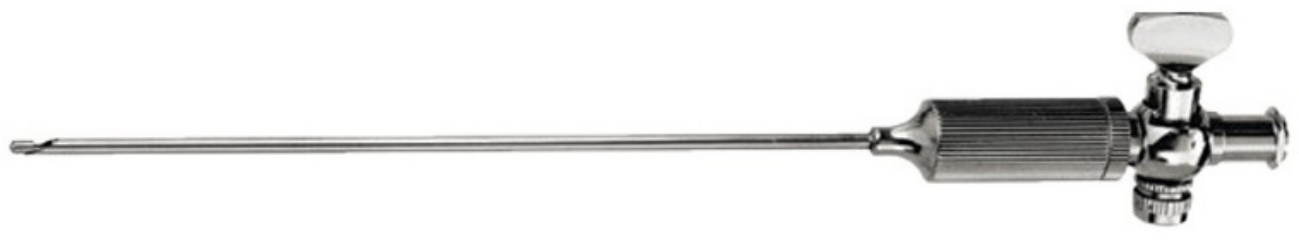

Figure 4. Veress needle (Stryker, MI, USA).

The open technique is an alternative method of creating a pneumoperitoneum. In this technique the peritoneal cavity is opened under direct vision [13] using a blunt-tipped trocar (Figure 5), the gas insufflator is connected to the trocar once it is inside the abdominal cavity. There are various techniques of open access like Hasson's technique, Scandinavian technique or Fielding technique.

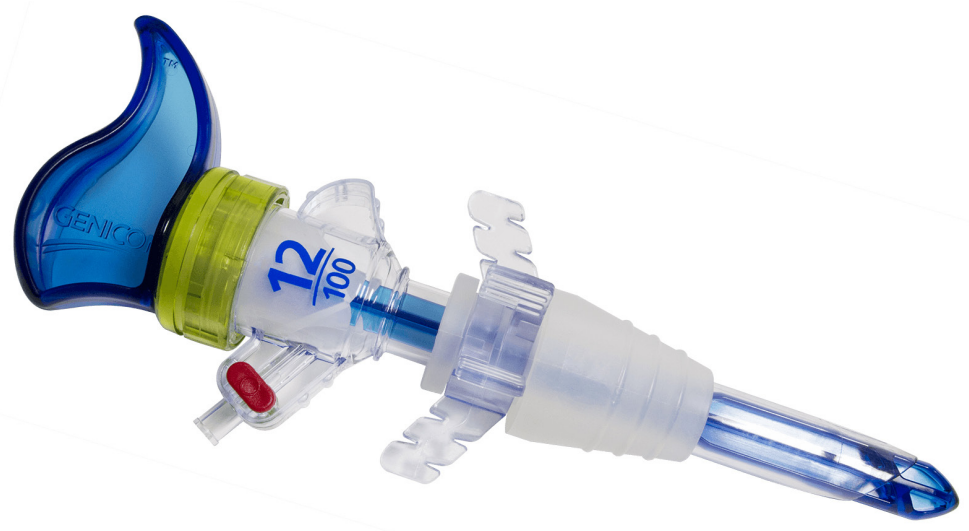

Figure 5. Hasson's blunt-tipped trocar (Genicon, Florida, USA). The Hasson's technique is one of the most commonly used ways to perform the open entry technique.

Open technique appears to be safer than closed method, but most laparoscopic surgeons prefer to use the Veress needle and insert the first trocar blindly, because they feel that the closed technique is faster, requires a smaller incision and is not associated with leakage of carbon dioxide [13]. 
Once the abdomen is insufflated by $\mathrm{CO}_{2}$, the surgeons can see the organs more clearly. Since 1982 a high-resolution video camera attached to a laparoscope is used to see the interior of the cavity [14]. This camera is inserted through the trocar, relaying images to a monitor located in front of the surgeon that gives a view of the abdominal interior.

This is the necessary procedure to complete an exploration of the cavity by laparoscopy. If a surgical treatment is required, secondary trocars are inserted through the abdomen to use diverse instrumentation. Inside the abdomen cavity, the instruments are used without direct vision, using the monitor image to carry out the required treatment. Once the procedure is complete the $\mathrm{CO}_{2}$ can be removed from the patient's abdomen and the incision closed.

\subsubsection{Laparoscopic conventional hand-held instruments}

Laparoscopy requires surgeons to develop the procedure from the exterior of the abdominal cavity. For this reason, they have to use long instruments that fit through the small ports of entry located in the abdominal wall.

The hand-held instruments used to perform these procedures have not vary enough since the mid 1980's. In rough outlines the instruments required to perform a laparoscopic procedure are: Veress needle, trocar, laparoscope, grasper and needle driver, as well as other specific instruments and devices required depending of the procedure.

The port of access to the abdominal cavity is called trocar (Figure 6). The trocar is an instrument composed by cannula and obturator. The obturator usually has a sharp point or a blade to facilitate the insertion of the trocar through the abdominal wall. Once inserted, the obturator can be extracted, keeping the cannula through the abdominal wall to allow access to the laparoscope or different instrumentation. 


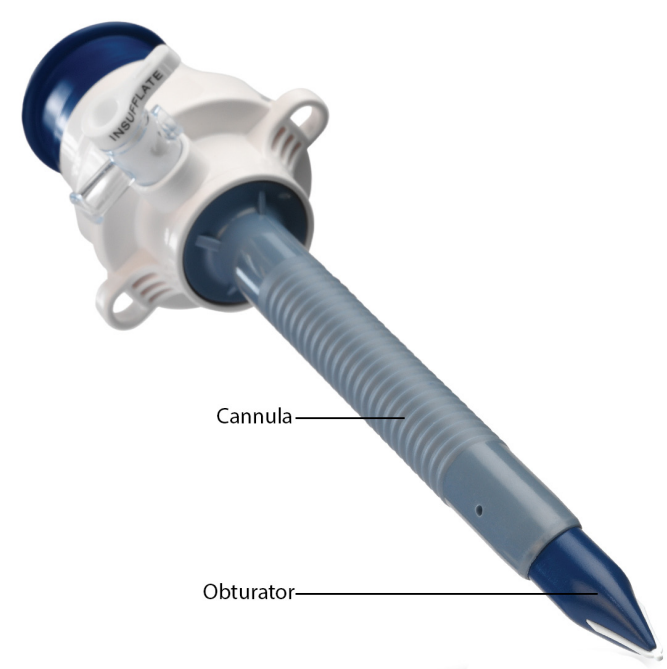

Figure 6. Trocar. Versaport Plus Bladeless Trocar (Medtronic, Minneapolis, USA).

Needle drivers or needle holders (Figure 7) are used to manipulate needles. It enables a free-hand suturing of wounds or surgical incisions within the body during laparoscopic procedures. Needle drivers have a blockage system to maintain the needle while the surgeon is preparing the area of suture.

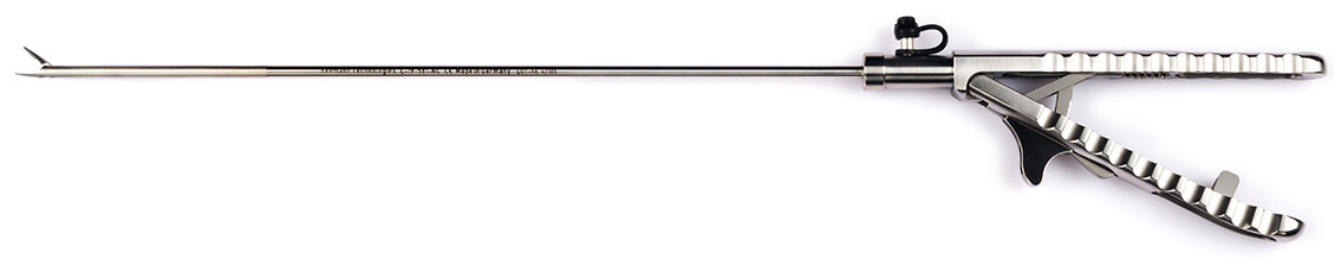

Figure 7. Needle driver (Matrix Surgical, Georgia, USA).

The laparoscopic grasping forceps (Figure 8) are grasping instruments designed to manipulate the delicate abdominal tissue during laparoscopic procedures. One of their main functions is to facilitate an adequate access to the surgical site by holding bowel tissue clear of the operating field. 


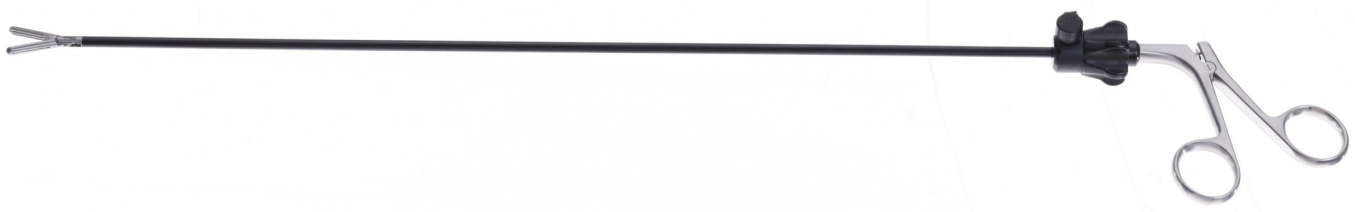

Figure 8. Laparoscopic grasper (Teleflex, North Carolina, USA).

The pistol-grip handle or ring handle configuration presented on the grasper is extended to a great number of instruments that perform different task during the surgery: cut, suture, coagulate or place stitches and clips.

\subsubsection{The pistol grip handle and the instrument tips}

The pistol grip handle configuration is in basic lines the presented in Figure 9. These instruments generally incorporate a 5 to $12 \mathrm{~mm}$ diameter shaft that houses the actuating mechanism and a rotating tip for tissue manipulation [7].

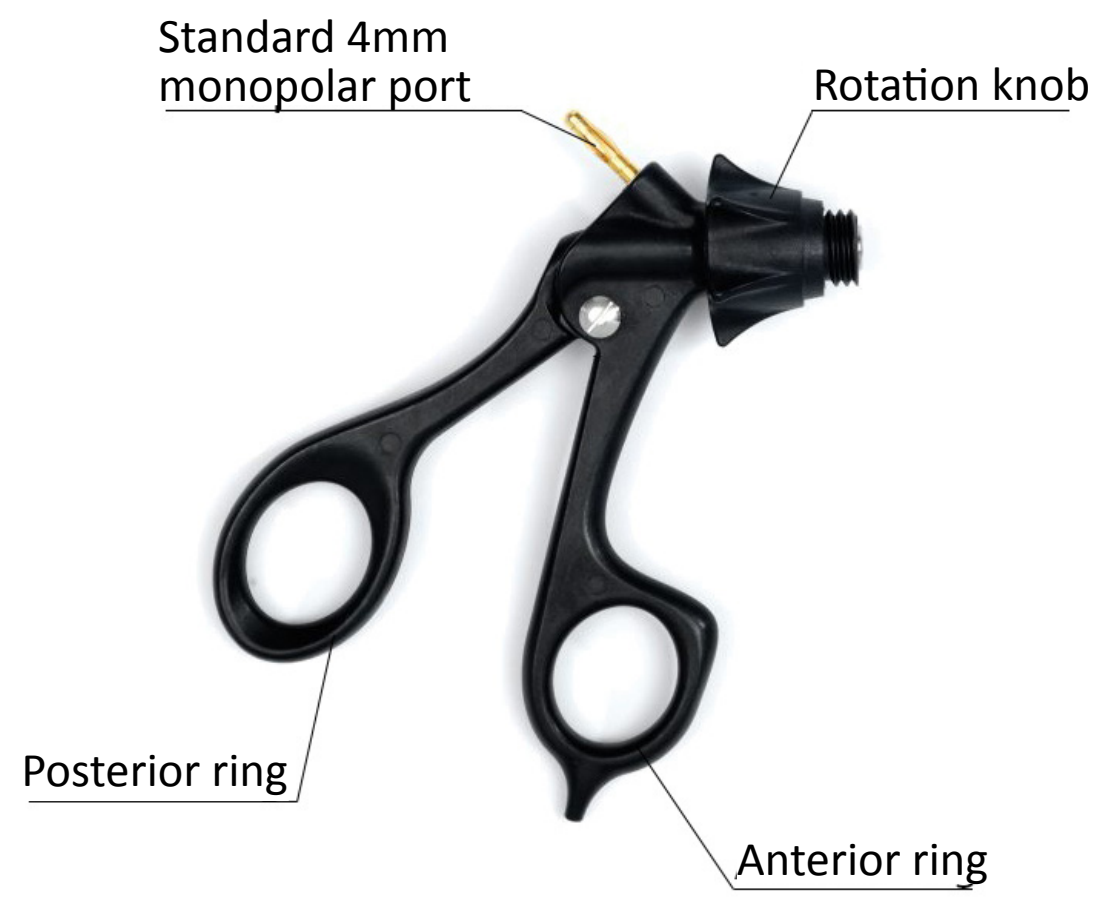

Figure 9. Conventional configuration of a pistol grip handle (Surgical Innovation, Leeds, UK). 
The instrument is grabbed with both hands indistinctly, the thumb is inserted in the posterior ring and commonly the middle finger in the anterior ring. The thumb is usually used to perform the strength over the other fingers. The index finger is used to move the rotation knob until the tip of the instrument in the right position. The monopolar port is used for electrosurgical procedures.

Different brands have different devices based in the same configuration. One of the most famous handles is the Snowden-Pencer (Figure 10). This instrument presents a thicker handle with padded rings to reduce the fingers pressure and a bigger anterior ring to introduce index and medium finger on it. The rotation knob of this instrument is bigger and has just four slots which make easier to rotate the tip during the procedure.

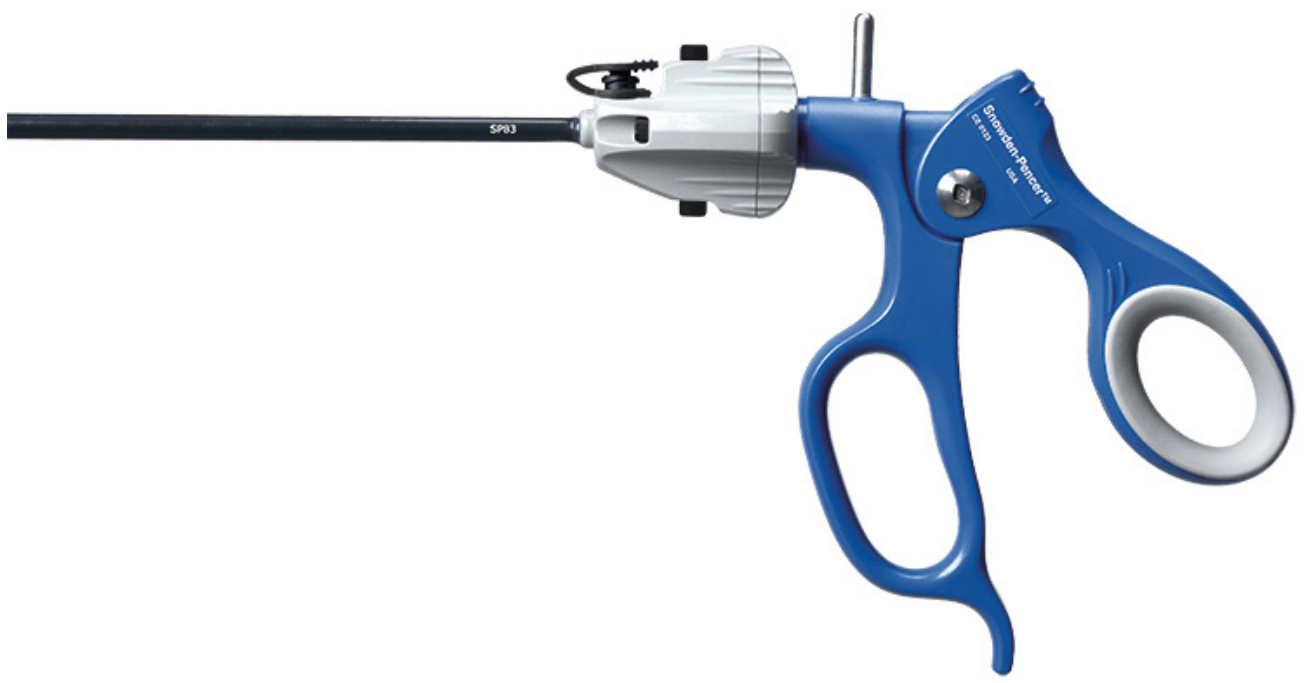

Figure 10. Snowden-Pencer (FarmPD, New Hampshire, USA).

The pistol grip handle configuration is used with different tips to configure different kind of instruments. The next paragraphs show some of the different tips that are used with this handle configuration. The use of each tip vary depending the moment of the procedure.

The grasping forceps are used to grasp and manipulate the abdomen tissue inside the abdominal cavity of the patient. Grasping forceps can be with single or double action jaw, all are traumatic to some extent. However, those with teeth or claws are very traumatic. The more pointed or narrowed jaws are more traumatic just like some spring loaded forceps [2]. 
The single action jaw graspers (Figure 11) are good when the surgeons do not have control over depth and they want to work in single plane in controlled manner. The grasping forceps with double action jaw (Figure 11) are the most commonly used by surgeons. The difference between single action and double action jaw graspers is that double actions can move both sides of the jaw, it allows larger grasp and blunt dissection.
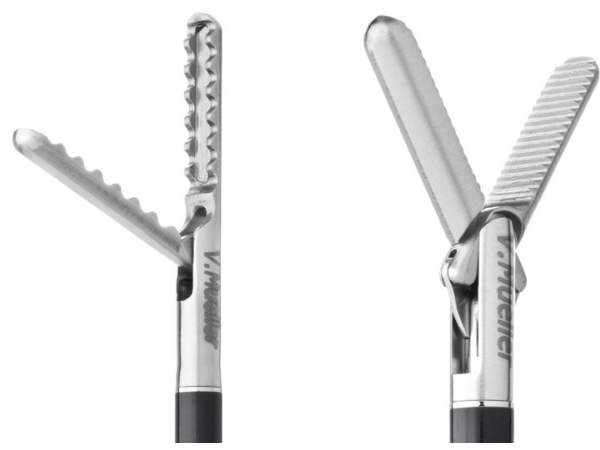

Figure 11. Single action jaw (left) and double action jaw (right) grasping forceps by BD (New Jersey, USA).

The Figure 12 present some examples of double side graspers. The variety is higher than in single side jaw and each one are created with a particular use.

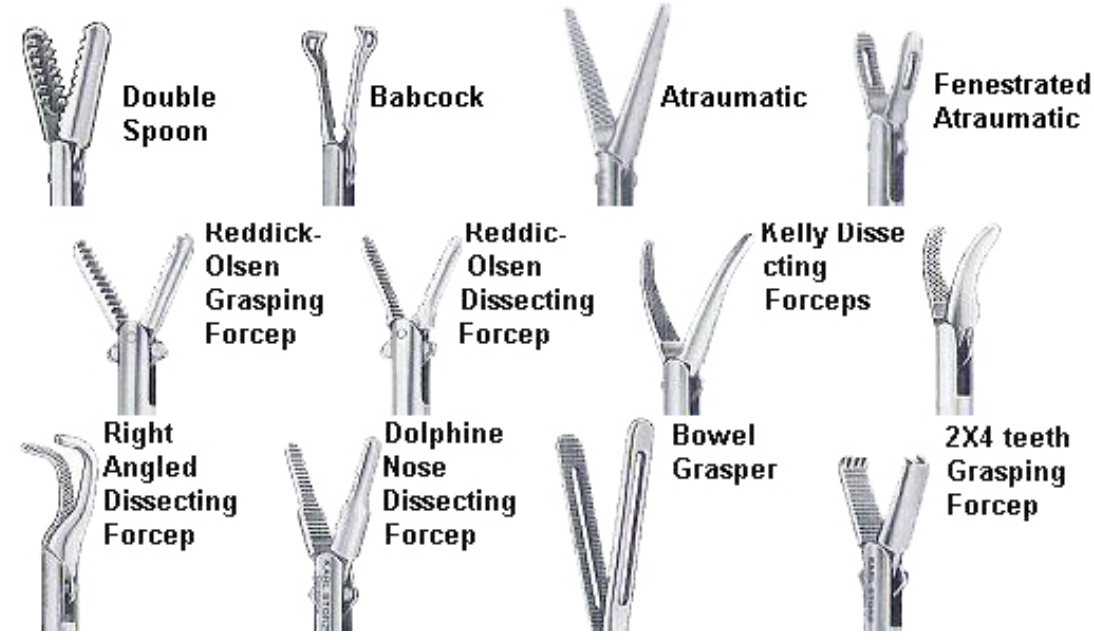

Figure 12. Different double jaw graspers on the market. Adopted from a document of the World Laparoscopic Hospital [15].

Scissors are used to perform many tasks in open surgical procedure but its use in laparoscopic surgery is restricted. In minimal access surgery scissors require greater skill because in inexperienced hand it may cause unnecessary bleeding and damage to important structures. 
In laparoscopy the surgeons can choose between different types of scissors depending on the use (Figure 13).

Curved scissors (Figure 13a) are multipurpose instrument but usually used for dissection with or without diathermy. To avoid accidental thermal injury to adjacent structures during diathermy dissection, especially in small operative spaces, the insulation sleeve should come right down to the moving blades [2]. Some surgeons prefer these scissors because its curvature of the blade abolishes the angle of laparoscopic instruments manipulation and allows a better view through the telescope.

The fine pointed curved or straight micro-scissors (Figure 13b) are usually used for micro-dissection or incisions into small structures such as the cystic duct or ureter [2].

Hooked scissors (Figure 13c) are appropriate for cutting ligated vessels and other tubular structures, as well as ligatures. They prevent accidental damage to nearby structures by lifting away the structures to be cut. They also prevent tissues and ligatures from slipping out of the jaws [2].

The straight scissor (Figure 13d) have the blade straight and is useful to cut pedicles and sutures [2].
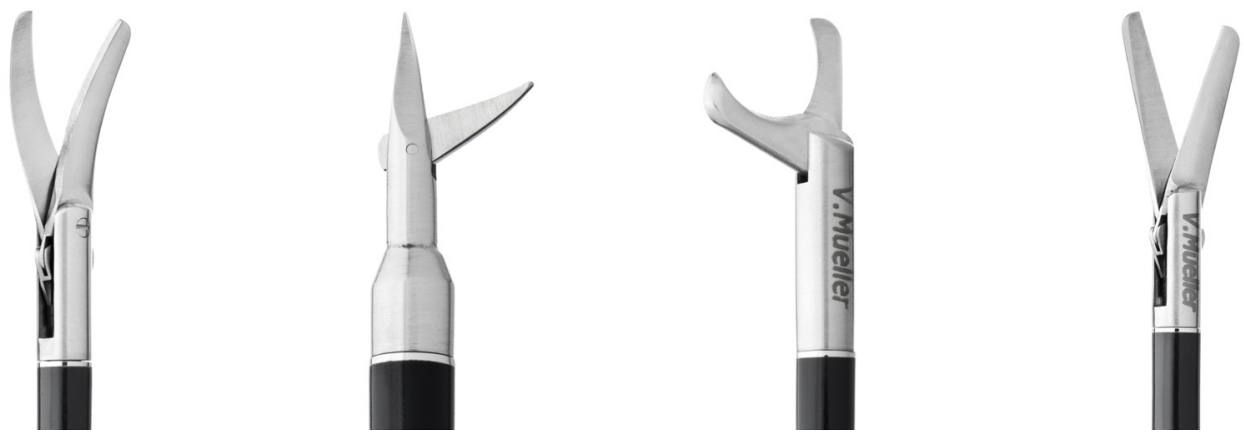

Figure 13. a) Double action curved scissor, b) Single action curved micro-scissors by $B D, c)$ Single action hooked scissors by BD, and d) Straight double action blade without serration by BD (New Jersey, USA). 


\subsection{Advantages of laparoscopy}

The advantages of laparoscopic procedures for the patient are countless and it is extensive the literature about this topic. This manuscript is focused in the different drawbacks that this procedure have for the surgeon. For this reason, the advantages section only include a brief explanation of the benefits for the patient.

The small size of the incisions used in laparoscopy allow the operation to be carried out with minimal trauma, with the avoidance of exposure, cooling, desiccation, handling, and forced retraction of abdominal tissues and organs [2]. It reduces the blood loss, the risk of infections and the operation pain [3]-[5]. The overall traumatic assault on the patient is reduced drastically (postoperative pain, ileus and wound complications), it also accelerates the recovery time and avoids the abdominal adhesion, which is cause of recurrent pain, intestinal obstruction, and female infertility [2].

The recovery time is an important advantage on laparoscopy. After an exploratory laparotomy the patient needs 2 or 3 days in the hospital to start eating and drinking normally and the complete recovery requires about 4 weeks. In laparoscopy this time is reduced, the time in the hospital is about a day and the complete recovery requires about a week. However, the recovery time depends on the treatment. Minor surgeries requires about two weeks in laparotomy and this time is reduced to few days by laparoscopy procedure [16]. But in major surgeries the difference increases being about 7,5 weeks required after the open procedure in contrast to 2 weeks after the laparoscopic procedure [17].

\subsection{Disadvantages of laparoscopy}

There are three different subjects that on which the laparoscopic has effect and the disadvantages are different: hospitals, patients and surgeons. 
- The disadvantages of laparoscopy for the hospital include the expensive equipment [2]. Not all hospital operating rooms can afford to offer it, because of the cost of purchase and maintenance of the equipment. Laparoscopic procedures require more technical expertise and take longer, at least initially, than an open approach [2]. Surgeons need to take special training in performing the many operations that are available by this means. The need for additional training is because laparoscopic surgeons leave the familiar territory of a three dimensional operating field to working on a two dimensional flat video display. The shift is a critical one, and requires some degree of practice moving around long laparoscopic instruments while handling delicate tissues. Despite these temporary disadvantages, with the proper training, surgeons are able to adapt to this means of operating.

- For the patient, the main difficulties with laparoscopy emanate from the necessity to insufflate the peritoneal cavity or extra-peritoneal space with gas, and access the space via needle and trocar inserted through the abdominal wall [2]. Occasionally, patients may get air pockets under the diaphragm. These pockets of air may cause neck and shoulder pain, which can be uncomfortable. Some patients complain of feeling bloated after this type of surgery. Usually, the pain associated with air pockets and sensation of bloating improves in the two to three days after surgery. Also exist the potential to injury to the vessels and viscera as the result of the insertion of the Veress needle and the trocar, inappropriate instrumentation and diathermy burns [2]. In addition, the laparoscopic surgery cannot always be performed on everyone, some patients with many prior operations may have scar tissue within the body that do not allow a safe procedure. It also required that patients undergo a general anesthesia or be "put to sleep", which means patients are hooked to a breathing machine during surgery. This may cause a sore throat after the operation.

- In regards to the surgeon, the difficulties include eye and hand coordination and the remote nature of the surgical manipulation, loss of direct hand manipulation and tactile feedback and the two-dimensional image provided by the current camera systems [2]. The axial skeletal posture is more upright during laparoscopic surgery as compared with open surgery which is accompanied by substantially less body and neck movement, less weight shifting, and more frequent awkward repetitive motions of the upper extremities [18], [19]. 
Shoulder motion is affected by fixed access ports, instrumentation design, and operating table height leading to more shoulder abduction compared with open surgery [19]. The effect of elongated laparoscopic instruments and the fulcrum effect at the abdominal wall is documented to require 4 to 6 times more strength in the forearm and thenar muscles than needed to complete a similar open surgical task [20], [21].

\section{Fatigue and musculoskeletal disorders associated to laparoscopic surgery}

Because this work is centered in the problems that laparoscopic procedures caused in surgeons' musculature, the next step is to review the concepts of ergonomic, musculoskeletal disorders and fatigue and how those affect the laparoscopic surgeons.

\subsection{Fatigue}

The term fatigue is too general and creates quite controversy. According to De Luca [22], the mention of the word fatigue to a group of health specialists and life scientists generate diverse and divergent descriptions because the concept of fatigue as applied to monitoring or measuring the deterioration of a performance of the human operator has been ambiguous and often misapplied. The first author which proposed the abandon of the word fatigue because its generality was B. Muscio [23] in 1921. Following the Muscio approach, in 1943 A.G. Bills [24] subdivide the concept of fatigue into subsets, identifying three major categories that correspond to different concepts of fatigue:

- Subjective fatigue: characterize by a decline of alertness, mental concentration, motivation, and other psychological factors.

- Objective fatigue: characterize by a decline in work output.

- Physiological fatigue: characterized by changes in physiological processes. 
In this manuscript is evaluated the physiological fatigue, more specifically the "localized muscular fatigue" which is the physiological fatigue caused by sustained muscular contractions.

The localized muscular fatigue was termed by Chaffin in 1971 [25] with the aim to evaluate the relative fatigue due to various common sedentary work postures and arm loads, and provide recommendations to assist the physician to improve the workplace, tool designs and methods specifications in industries where physical stress caused significant decrease in the workers faculties.

This term is commonly used to denote an inability of a muscle or group of muscles to sustain the required or expected force [26]-[28] but it is also manifested by muscular tremors and localized pain [22].

Is common to understand the localized muscular fatigue as a consequence of the over exercise which occurs only after a period of time. Nevertheless, much evidences suggest that the physiological events underlying this fatigue begins at the onset of activity [26]. The discomfort associated with the muscle fatigue could be due to the intention of the Central Nervous System (CNS) of interpret an effort as discomfort to motivate relaxation of the contracted muscle [25]. Another explanation is that the metabolism within the muscle fibers produces a "pain induced metabolite", it happens because the metabolite diffuses out of its site of production in the muscle cells into the intercellular fluid. Then, the rate of production of metabolite and its diffusion into the extracellular space exceeds the rate at which it can be eliminated by the muscle, its increasing concentration at the nociceptors generates the sensation of pain [29].

Several interpretation explain why the localized muscular fatigue occurs. The simplest explanation is that one or several of the physiological processes that enable the contractile proteins to generate a force become impaired [30], but this is the more simplistic view. The complex one understand the term fatigue as a concept dependent of a class of effects that impair the motor performance. This approach understands the fatigue as a concept that could produce changes around four main topics [31]: 
- Task dependency: The specific mechanisms that cause fatigue in a given condition are determined by the details of the task performed by the individual. The task variables that influence on the muscular fatigue are the level of motivation of the subject, the intensity and duration of the activity, and the extent to which the activity is continuously sustained.

- Force-fatigability relationship: In general, greater the force exerted by a muscle for a given task means more muscle fatigue. This suggests that the mechanisms underlying fatigue scale with the force that is exerted.

- Muscle wisdom: One set of adaptations that has been associated with sustained activity includes a concurrent decline in force, relaxation rate, and motor neuron discharge. This association has been termed "muscle wisdom" because it optimizes the force and ensures an economical activation of fatiguing muscle by the CNS.

- Sense of effort: The effort associated with performing a task is assessed by requiring subjects to match forces. It seems that subjects' judgments are based on the effort required to generate a force rather than the absolute magnitude of the force that is exerted. This judgment is referred to as the sense of effort and is distinct from the force sensation associated with a contraction.

The muscle fatigue can be measured as a reduction in muscle force, a change in electromyography (EMG) activity or an exhaustion of contractile function [28]. By means of EMG signal analysis, localized muscle fatigue can be demonstrated even before the sensation of pain is evident [32].

\subsection{Fatigue associated to laparoscopic surgery}

In 1995 A. Cuschieri [33] coined a new characteristic implicit on the minimal access processes, the "surgical fatigue syndrome". Several authors evaluated the fatigue symptoms during laparoscopic surgery comparing the results with the conventional open laparotomy. The reports coincide that the postures during the practice are the mayor contributing factor in the surgeon's extreme muscle fatigue and chronic injuries [33]-[36]. 
The postures acquired during laparoscopic surgery are forced by the practice (Figure 14). As explained above, during a minimal access surgery the surgeon performs a procedure through a fixed port of access inserted in the abdominal cavity of the patient, this fixed port of access determines the manipulation angle between instrument, the elevation angle between the instrument and horizontal plane, and the angle between the instrument and optical axis of the endoscope [37], [38]. The instruments movements are in opposite direction to the tips movements, the length of the instruments requires the surgeons to use a large envelope of motion (Figure 14), more force is required to move or separate obstacles and also reduces the precision of their movements [34].

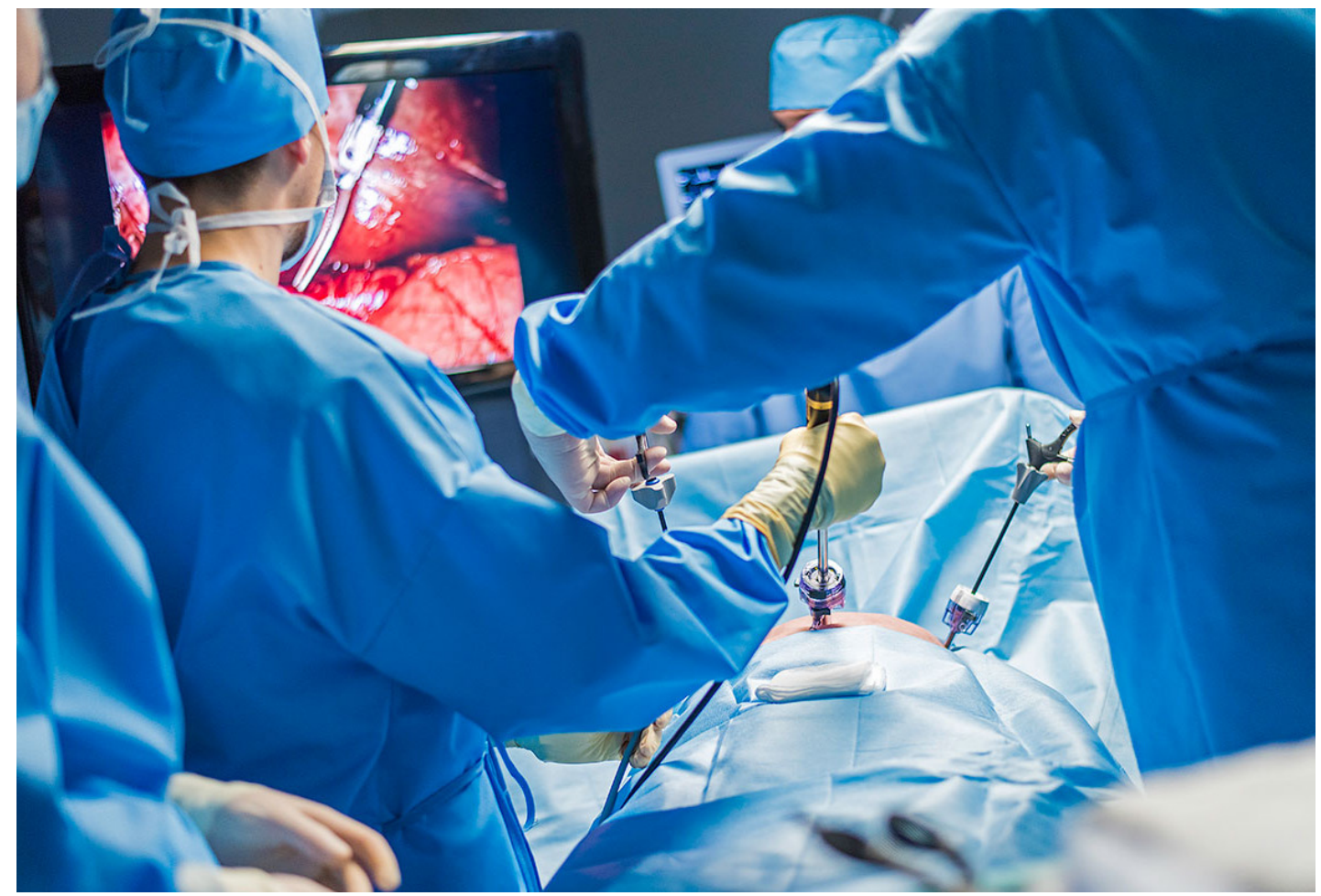

Figure 14. Surgeons posture during laparoscopic procedure.

All these issues create a combination that affect the surgeon's posture and the procedure, it makes that the performance of the procedure declines on average after 4 hours, thereafter the fatigue is manifested by mental exhaustion, increased irritability, impaired surgical judgment, reduced dexterity [33] and an increase of muscle tremors [25]. 
Pain, fatigue and stiffness are the symptoms most commonly reported by surgeons mainly in the areas of back, arms and neck [39], [40], but also is reported frequently pain or numbness in the wrists and hands because of the instruments [7], [41].

According to the ergonomic principles, if a discomfort or muscular fatigue, caused by a work related task is ignored, it will gradually become more intense and eventually may result in a musculoskeletal disorder, such as tendonitis, tenosynovitis, or serious nerve-compression injury like carpal tunnel syndrome [42]-[45].

\subsection{Musculoskeletal disorders}

The term musculoskeletal disorders refers to conditions that involve the nerves, tendons, muscles and supporting body structures [46]. Musculoskeletal disorders were recognized as a work-related condition as the beginning of the 18th century. Many work-related diseases have their origin in the exposition to a particular hazardous agents, but musculoskeletal disorders are characterized as multifactorial because physical, psychological, and individual factors contribute to its development and aggravation [47]. There is a wide range of ergonomic research methods used to evaluate the cause of potential musculoskeletal disorders categorized under three families [48]:

- Direct measurement is a type of ergonomic evaluation performed by sensors attached directly to the subject in order to measure different variables at work. Some examples are: Lumbar Motion Monitor (LMM) technique, that is used to evaluate the back posture and motion by an electronic goniometer; Inclinometers that are used to measure the postures and movements of the head, back and upper limbs using several accelerometers that record the movement in two degrees of freedom (DoF) with reference to the line of gravity ; or the analysis of the EMG activity which can be used to estimate variations in muscle tension [49] and to evaluate localized muscular fatigue [22], [50]. Direct measurement systems can provide large quantities of highly accurate data on a range of exposure variables. The sensors directly attached to the body may cause discomfort and possible some modifications in work behavior. This system requires a considerable initial investment to purchase and maintain the equipment and is time-required for the analysis and interpretation of the data [48], [51]. 
- The observational methods are non-invasive techniques used to measure the risk of musculoskeletal disorders that a worker has in the workplace. These methods have the advantages of being inexpensive and practical for use in a wide range of workplaces where using other methods would be difficult [48]. To record the task is used a technique called Motion Capture (MoCap). This technique is used to record the movements of a human or animal characters in orders to use it in different industries as cine, video games, or biomechanics evaluation [52]. Once the work-task is recorded is necessary to evaluate the movements and there is a number of techniques used to evaluate it, for example: OWAS (Ovako Working Posture Analyzing System) is a technique used to measure the whole body posture; RULA (Rapid Upper Limb Assessment) is used to assess the upper body and limbs; NIOSH (National Institute of Occupational Safety and Health) is a measurement method to identify musculoskeletal risk factors; or REBA (Rapid Entire Body Assessment) technique used for asses the entire body during dynamic tasks [48].

- The self-reports include worker diaries, interviews and questionnaires and collect data on workplace exposure to physical and psychosocial factors [48]. Generally, this method uses large samples of written record but also includes self-evaluation of video films during a work-task and webbased questionnaires. The advantages of these methods is that they are straightforward to use, applicable to a wide range of working situations and appropriate for surveying large numbers of subjects at comparatively low cost. Also this method contemplate results of exposures to riskfactors for longer periods than may be expected by making observations at the workplace [48].

The choice of method depends of the nature and purpose of the investigation for which de data will be used. In health care, the relevance of ergonomics evaluations increase because of the potential for harm a patient [53]. Surgeons exposed to poor ergonomic postures during long term periods present mental exhaustion, irritability, impaired surgical judgment, low dexterity and muscle tremors [25], [33]. 


\subsection{Ergonomic and musculoskeletal disorders associated to laparoscopic surgery}

In the operation room, the ergonomic is defined as the study of the surgeon in relation to their working environment, based on anatomy, psychology and engineering, combined in a systems approach [54] and including topics as the range of movement of parts of the human body and the design of instruments [55].

Laparoscopic surgeons are a group having high risk of having work-related musculoskeletal disorders[56]. Laparoscopic surgery has changed the interaction of the surgeon in the operative field and hence has changed the body posture and upper extremity movements of the surgeon [41].

In 1993, Tendick [57] was the first author which thoroughly discussed the manipulation problems in laparoscopic surgery, highlighting the negative effects on the surgeon's dexterity of the limited DoF during the use of laparoscopic instruments. In 1995, further reviews of human interface problems in endoscopic surgery was performed, identifying the need of an ergonomic approach to design the laparoscopic operating environment [54].

In 1999 a survey collected the answers about the body part discomfort of the surgeons at the Society of American Gastrointestinal Endoscopic Surgeons (SAGES) [20], showing that almost $12 \%$ of the surgeons reported frequent pain or numbness in the neck, shoulder, arm and wrist. In the years before this digit increased, reaching values near to $40-60 \%$ in 2006 [42] and to more than $80 \%$ in the most currently performed surveys [58]-[60]. Despite the real value, the evidence is that the static muscle loading, the repetitive fine motor handling techniques, the extreme joint angulations and the work duration required by the laparoscopic procedures are risk factors for developing musculoskeletal disorders [21]. 
One of the most recognized physical stress factors in the surgeon's work is the long duration of maintaining awkward static postures during surgery [56]. This is the reason which the majority of the surgeons stated that ergonomic is important in the operation room [58]. The goal of proper posture is comfort, efficiency of movement, and minimization of the risk of musculoskeletal injuries to the operator [6]. The surgeon's neck and back should be maintained in a comfortable and upright position facing forward. According to R. Berguer [61], during laparoscopic surgery, the ability to achieve this ideal posture is determined by the height of the operating room table, the position of the visual display, the foot pedal locations and the hand instruments.

\section{The design of ergonomic laparoscopic hand-held instruments}

During laparoscopic surgery, the surgeons indirectly views the operative field and can only touch the intra-abdominal tissues with long instruments via ports that are placed in fixed locations. Their senses are working much harder to achieve the same goals than in conventional open surgeries, for this reason, the proper design of the instruments and the layout of the operating room is critical to avoid fatigue and human errors [6].

Although the surgeon's comfort is not the primary focus of the operations, the data reported by Hanna et al. indicate that inefficient working postures directly affect his or her work efficiency [38]. It is also well known that the incidence of workrelated musculoskeletal injury is directly proportional to workplace hazards such as excessive muscle loading [62], [63].

According to Berguer [61], no single laparoscopic instrument design is substantially superior to others, so each surgeon needs to choose the design that best achieves the following goals: 
- Enables the surgeon to keep both wrists in a neutral position.

- Permits the surgeon to keep both arms at the sides of their body.

- Avoids pressure points on the hands.

- Allows the surgeon to apply force with a power grip hand position.

- Allows fine manipulation with a precision grip hand position.

\subsection{Requirements for an ergonomic laparoscopic handle}

In 1998, U. Matern and P. Waller [64] presented the principles of ergonomic handles for instruments used in minimally invasive surgery. The authors presented interesting results as the most important functional zones of the palm for manipulating laparoscopic handles (Figure 15) because most of the mechanoreceptors of the tactile sense are located in these areas and not equally distributed over the surface of the hand or the minimal demands that a laparoscopic instruments need to be functional, comfortable and ergonomically satisfactory.

According to the author the handle should allow several functions and has to be adjustable for various hand sizes, being as small as possible while the elements size allow easy manipulation. It also should allow one-handed use and the function should be ascertainable from the design of the handle. Its functional elements must be easily accessible and should be operated by the sensitive areas of the hand, avoiding pressure areas in the hand.

Any necessary springs must function adequately and should in no way hinder use, indirect power transmission with loss of power is to be avoided. The instrument should have minimal autonomous dynamics (artificial movements). Cramped positions as well as excessive shoulder movements are to be avoided. Finally, the instrument's shaft must be an extension of the forearm rotation axis [64]. 


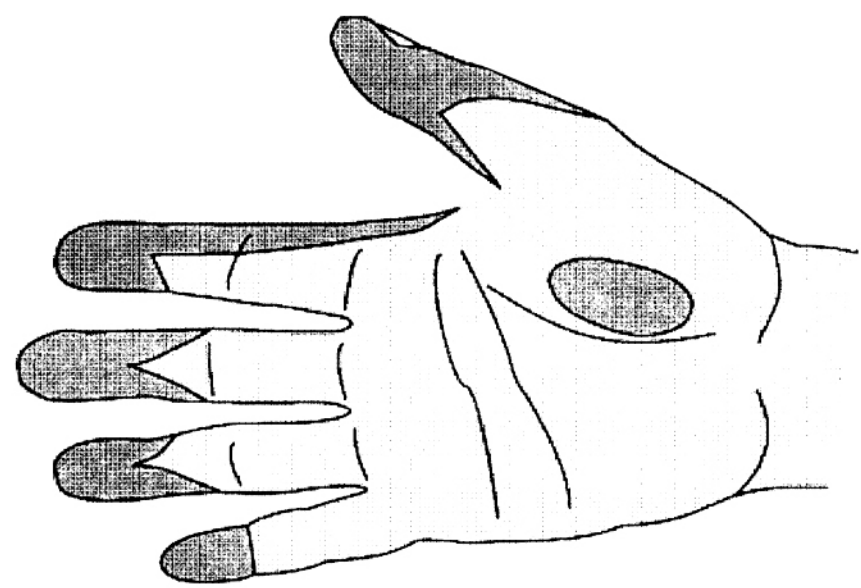

Figure 15. Most important functional zones of the palm for manipulating laparoscopic handles. Adapted from [64].

Some years later, in 2001, an important study presented by Van Veelen et al. [65] presented a new list of ergonomic design criteria for handles of dissection forceps. The authors decided to develop this new list because they thought that the general criteria used to evaluate the handle design had a few shortcomings to evaluate the dissection forceps and its use in laparoscopy. In Table 1 are presented the general requirements for laparoscopic handle design and the specific new ergonomic requirements for dissection forceps handle design presented by Van Veelen.

Table 1. General and proposed ergonomic requirements for hand tools and dissection forceps. Adapted from [65].

\begin{tabular}{|c|c|c|}
\hline & General requirements & $\begin{array}{l}\text { New ergonomic } \\
\text { requirements }\end{array}$ \\
\hline \multirow[t]{3}{*}{ Posture of hand and arm } & $\begin{array}{l}\text { Angle between handle } \\
\text { and tube must be } \\
\text { between } 14^{\circ} \text { and } 24^{\circ}\end{array}$ & $\begin{array}{l}\text { Angle between handle } \\
\text { and tube must be } \\
\text { between } 14^{\circ} \text { and } 50^{\circ}\end{array}$ \\
\hline & & $\begin{array}{l}\text { When handle is } \\
\text { manipulated with } \\
\text { precision grip, wrist } \\
\text { excursions must be } \\
\text { neutral in } 70 \% \text { of } \\
\text { manipulation time }\end{array}$ \\
\hline & & $\begin{array}{c}\text { When handle is } \\
\text { manipulated with force } \\
\text { grip, wrist excursions } \\
\text { must be neutral in } 70 \% \text { of } \\
\text { manipulation time }\end{array}$ \\
\hline
\end{tabular}




\begin{tabular}{|c|c|c|}
\hline & General requirements & $\begin{array}{l}\text { New ergonomic } \\
\text { requirements }\end{array}$ \\
\hline \multirow[t]{3}{*}{ Forces in hand and arm } & $\begin{array}{c}\text { Grip opening must be } \\
\text { between } 60 \text { and } 90 \mathrm{~mm}\end{array}$ & $\begin{array}{c}\text { Grip opening must be } \\
\text { between } 60 \text { and } 80 \mathrm{~mm}\end{array}$ \\
\hline & $\begin{array}{l}\text { Forces for opening and } \\
\text { closing tip must be } \\
\text { produced by flexors of the } \\
\text { hand/fingers; this can be } \\
\text { accomplished by use of } \\
\text { spring for opening tip }\end{array}$ & $\begin{array}{c}\text { Any disturbances such } \\
\text { as friction and spring } \\
\text { forces must be avoided } \\
\text { to bring about optimal } \\
\text { force feedback of tissue to } \\
\text { surgeon's hands }\end{array}$ \\
\hline & $\begin{array}{l}\text { Forces for manipulating } \\
\text { instrument must be as } \\
\text { low as possible }\end{array}$ & $\begin{array}{c}\text { If handle is manipulated } \\
\text { in free spaces, no friction } \\
\text { must be experi-enced }\end{array}$ \\
\hline $\begin{array}{l}\text { Compressive force on the } \\
\text { hand }\end{array}$ & $\begin{array}{c}\text { Handle must have a large } \\
\text { contact area to prevent } \\
\text { extreme contact-area } \\
\text { pressure }\end{array}$ & $\begin{array}{l}\text { Handle must have } \\
\text { minimum width of } 10 \\
\text { mm to prevent extreme } \\
\text { contact area pressure }\end{array}$ \\
\hline Finger move-ment & $\begin{array}{l}\text { Control switches must be } \\
\text { manipulated with thumb }\end{array}$ & $\begin{array}{l}\text { Instrument must be } \\
\text { provided with knob to } \\
\text { allow rotation tip. This } \\
\text { con-trol switch must be } \\
\text { manipulated with thumb } \\
\text { or forefinger, and when } \\
\text { manipulated in free } \\
\text { spaces, no fric-tion must } \\
\text { be experienced }\end{array}$ \\
\hline Left-handed & $\begin{array}{l}\text { Handle must allow } \\
\text { left- and right-handed } \\
\text { manipulation }\end{array}$ & $\begin{array}{l}\text { Handle must allow } \\
\text { left- and right- handed } \\
\text { manipulation }\end{array}$ \\
\hline Anthropometry & $\begin{array}{l}\text { Dimensions of finger rings } \\
\text { must be: inner length } \\
\text { mini-mally } 30 \mathrm{~mm} \text {, inner } \\
\text { width minimally } 24 \mathrm{~mm}\end{array}$ & $\begin{array}{l}\text { Dimensions of finger rings } \\
\text { must be: inner length } \\
\text { minimally } 30 \mathrm{~mm} \text {, inner } \\
\text { width minimally } 24 \mathrm{~mm}\end{array}$ \\
\hline
\end{tabular}

These criteria were created in order to help the companies in the manufacture of optimal pistol-grip handles for laparoscopic surgery and should be take into account during the ideation process in order to make useful and ergonomic prototypes to test. Once the design is considered optimum, the decision of selecting a prototype for human factors testing entails a dilemma. A prototype of too high fidelity is very time-consuming and expensive to build. But the findings obtained with a prototype of too low fidelity may not be valid. This requires the careful consideration of what level of fidelity would be more adequate for the test [66], making useful to have different prototypes to use under different conditions. 


\subsection{Solutions presented by other research teams}

Universities and research centres of all over the world are trying to found solutions that improve the different lacks of the conventional laparoscopic instruments. The products and prototypes presented here are just the most relevant of the wide variety of solutions, but the relation new devices-publications is not really big because generally this innovations are rapidly patented by a company and included on the market to be exploded.

Prof. Sir Alfred Cuschieri and his team are well-known in the field of ergonomics and improvement of hand-held instruments for laparoscopic surgery. His team developed handles as Ball handle (Figure 16) or Rocker handle (Figure 17) for endoscopic surgery among others.

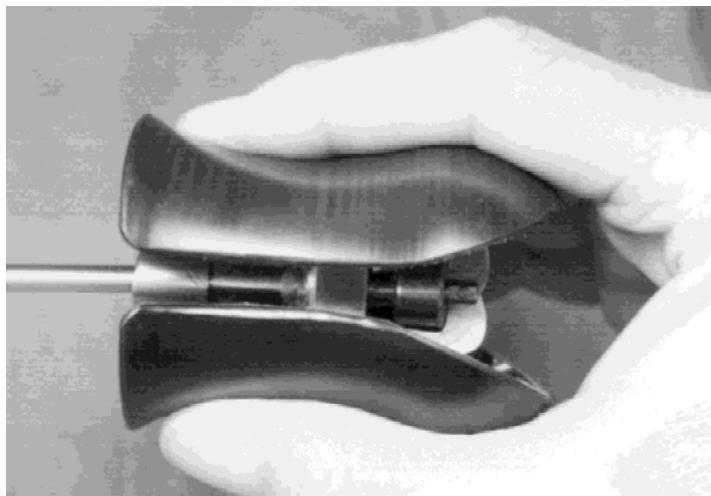

A

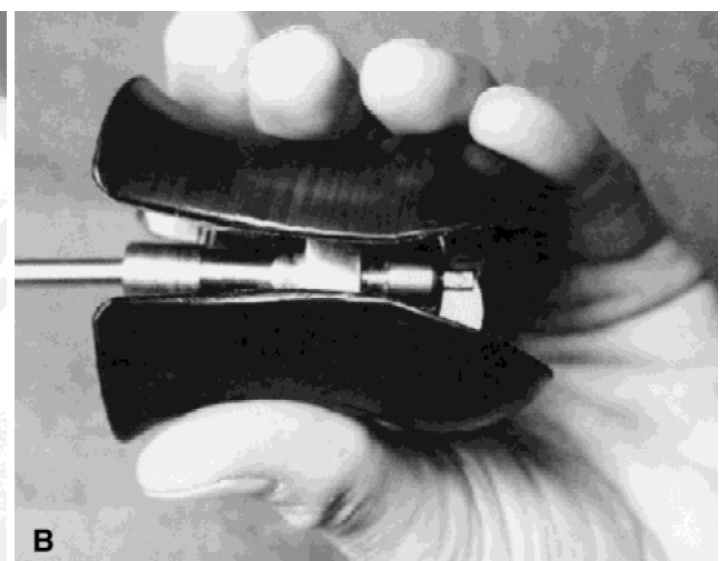

B

Figure 16. Prototype ball handle needle driver. A) Line grip and B) transverse grip.

Figure adapted from [32].

The ball handle lies comfortable in surgeon's palm. The design reduces the fatigue of surgeons and facilitate the rotation of the instrument by allowing rotation within the palm rather than using wrist rotation. Making pressure on the front of the handle with the thumb and the first fingers the jaw of the instrument is closed and making pressure in the rear of the handle, the jaws are open. 


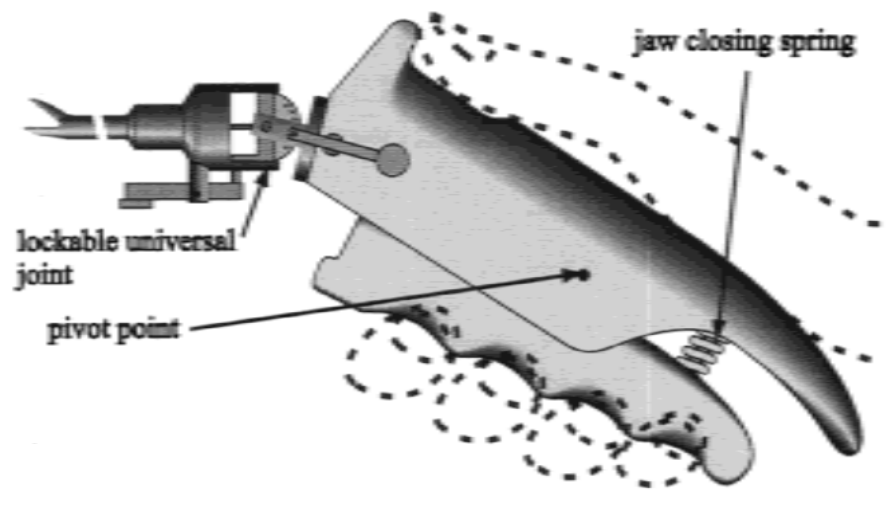

Figure 17. Design of the Rocker handle needle driver. Figure adapted from [67].

The Rocker handle also has very great ergonomic value especially when used with needle holder. This handle allows the surgeon to change the handle-to-shaft angle in order to reduce the surgeon's wrist angle. The angle can be changed pressing the angle latch even during the procedure. The jaw of the instrument is closed pressing the front part of the handle.

Intuitool (Figure 18) is probably one of the most famous projects that tries to reduce the musculoskeletal problems during laparoscopic procedures. This device was developed by the Prof. Hallbeck's team on the University of Nebraska. It is an articulating grasper with intuitively controlled handle. The handle design allows surgeons to operate the Intuitool in multiple positions. It is obvious to grasp and the surgeon intuitively understands how to articulate and close the grasper based on his hand position. When the user moves the control sphere forward, the grasper articulates up. Similarly, the grasper articulates left, right or down when the Surgeon moves the control sphere left, right or back [68]-[71].

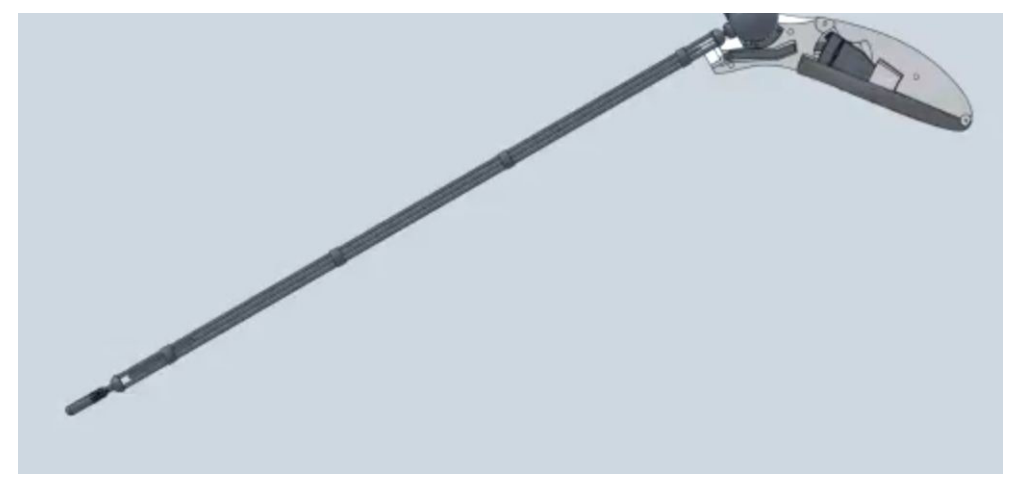

Figure 18. Prototype Intuitool. Developed by the University of Nebraska. 
FlexDex (Figure 19) is an instrument developed by the Precision System Design Laboratory at the University of Michigan and commercialized by FlexDex Surgical. FlexDex is a mechanical hand-held instrument that, according to the authors [72] "establishes for the first time the feasibility of achieving enhance dexterity, intuitive and ergonomic control, and force feedback in a simple mechanical tool". FlexDex increases the manual dexterity, increases support of the surgeon's hand and allows the surgeon to perform more difficult stiches inside the body avoiding the cost and complexity of robotically controlled laparoscopic procedures.

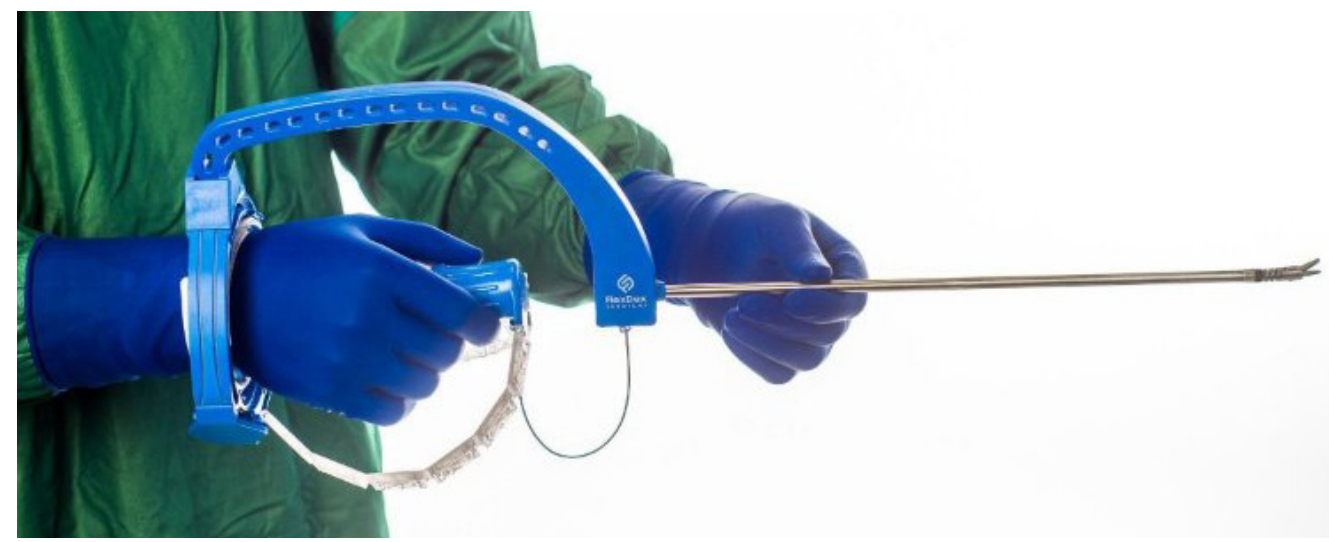

Figure 19. FlexDex instrument (FlexDex Surgical, Michigan, USA).

Maestro (Figure 20) is a laparoscopic instrument developed by the Vanderbilt University. It is a non-robotic dexterous laparoscopic manipulator with a wrist providing seven DoF. The surgical manipulator offers surgeons a dexterous hand which can be used in procedures that would normally require the use of rigid laparoscopic tools. Despite the large amount of alternative laparoscopic instrument handle configurations proposed by different research teams, except in the case of the Intuitool, which team evaluated the usability, the buttons location, and the position reached during different exercise, the ergonomic data to support their use are still lacking [7].

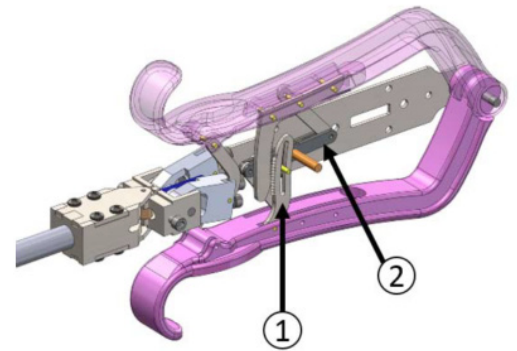

(1)

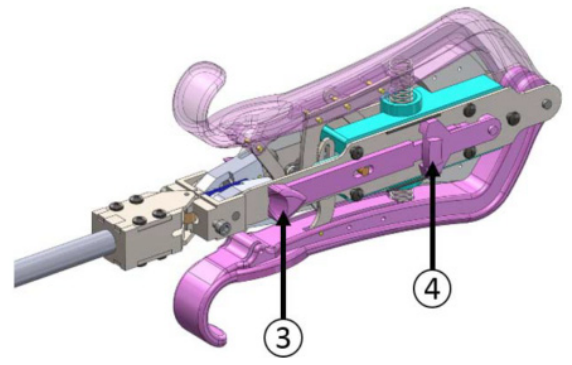

Figure 20. Maestro instrument. Figure adapted from [73]. 
In this manuscript, the approach used is new and as far as our knowledge it never has been used before. In this manuscript, a new concept called "Postural Freedom" has been evaluated, which was coined for this work purposes. This concept could be a key element to reduce the musculoskeletal problems associated to laparoscopic surgery and, during the next sections, this will be tested and evaluated by means of different experiments to confirm its benefits for the surgeon 



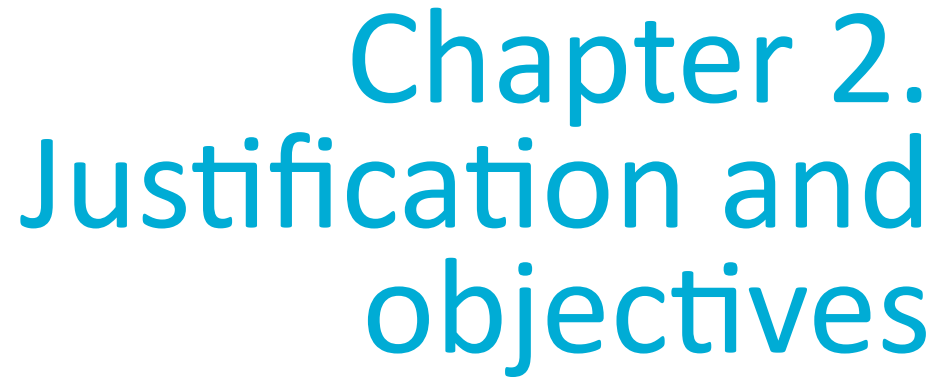




\section{Justification}

\subsection{Key points that justify this manuscript}

Laparoscopic surgery and MAS are going to be the standard in the near future. These procedures are clearly safest for the patient than conventional ones and reduces their hospitalization time. The ergonomic lacks and diverse difficulties that instrumentation and procedures pose to surgeons has to be fixed in order to increase the amount surgeries performed by minimally access procedures.

The percentage of surgeons suffering pain, numbness and muscular fatigue is considerable (80\%) and increasing year on year [20], [58]-[60]. Their exposition to poor ergonomic postures during long term periods present mental exhaustion, irritability, impaired surgical judgment, low dexterity and muscle tremors [25], [33] which contribute to technical errors.

According to the literature, there are serious complications for the patient resulting from technical errors during laparoscopic surgery. This errors occur mainly during the early learning phase and caused one half of the operative deaths [74]. Those facts indicates that is imperative to reevaluate the current design of laparoscopic handles in order to reduce it effect on surgeons' body.

The next sections present studies of surface electromyography (sEMG) and MoCap, in order to identify the effect that different handle designs have over surgeons' musculature and over the amount of time in neutral postures during the procedure.

In addition, the last section introduce "Evotool", an instrument patented by the Universitat Politècnica de Valéncia and the Hospital La Fe de Valencia that could reduce the negative impacts caused by conventional instruments. 


\subsection{The concept of "Postural Freedom"}

The poor understanding of ergonomic guidelines in laparoscopic surgery added to the conventional laparoscopic instruments which have the transmission rod fixed to the handle, forces surgeons to move their upper limb into uncomfortable positions [32], [33], [41], [61], [75], [76].

This research aims to present a new design solution that reduces the uncomfortable positions in laparoscopic surgery and the problems that arise from it. The base hypothesis used in this research is as follow "if you allow people to work without restrictions in laparoscopic surgery, they adopt a more beneficial, ergonomic position for their musculoskeletal system and their movement and posture are closer to the neutral positions". In this context, it is introduced the term Postural Freedom (PF) to refer the characteristic and the different elements that allow surgeons to adopt unrestricted movements during laparoscopic procedures.

Contrary to the conventional laparoscopic instruments that have the handle fixed to the transmission rod, an instrument with a PF characteristic allows the movement and the spin relative to both elements. This characteristic has the potential to increase the neutral positions in upper body during laparoscopic surgery and could be the key to reduce the musculoskeletal disorders.

\section{Objectives \\ 6.1. General objectives}

The premise of this work is that current laparoscopic instruments produce adverse effects, as pain, stiffness, and muscular fatigue in surgeons' upper body. These adverse effects are caused by the prolonged non-neutral postures maintained during the procedure. Which force the surgeons to displace their upper limbs, reaching critical positions. 
The consequences are mainly suffered by the surgeon but, this constant exposition to poor ergonomic postures during long term period contribute to technical errors as muscular tremors and impaired surgical judgment that may harm the the patients.

For this reason, it is necessary to improve the ergonomics in the operating room, in particular improving the instruments that force the surgeons to reach critical positions during the procedure. In order to improve those instruments, the concept of PF is applied in the design and manufacture of a prototype that released the surgeons of forced positions. These forced positions are mainly caused by the fixed handle-to-shaft angle provided by the conventional instruments. The new design presented in this manuscript has a variable handle-to-shaft angle that allows surgeons to avoid those critical positions.

Thus, a summarized objective is to demonstrate the postural health provided by a laparoscopic instrument that do not force the surgeons' movement. It is a multidisciplinary approximation for an ergonomic problem detected some decades ago that increased recently for the increasing number of laparoscopic procedures developed worldwide.

Two different techniques are used in order to evidence the improvements caused by the PF concept. On the one hand, the SEMG by capturing the signal on the muscles affected and using different techniques to processed the signal. And on the other, the MoCap by the video recording of the movements and processing each displacement with a specific software. If finally the effect of the PF is demonstrated, a final design would be developed in order to be tested by surgeons.

Therefore, three general objectives are planted, accordingly with the three lines explained:

- Objective G1. Demonstrate the reduction of muscular fatigue in relevant muscles of upper body with an instrument based in the concept of "Postural Freedom". The technique used to evaluate the muscular fatigue is the surface electromyography. The experiment simulates a set of positions commonly performed during real procedures.

- Objective G2. Demonstrate the reduction of critical positions in upper 
limbs with an instrument based in the concept of "Postural Freedom". The technique used to evaluate the position of the upper limbs is the Motion Capture. The experiment replicates a traditional test used to evaluate new instruments in laparoscopic simulations.

- Objective G3. Design and prototyping of an instrument based in the concept of "Postural Freedom" and evaluate it usability with real users.

\subsection{Specific objectives}

In order to achieve each general objective, a series of objectives more specific are defined:

- Objective G1.1. Create a database of electromyography registers in muscles trapezius, deltoids, biceps and flexor carpi radials. The registers will be obtained while the participants use two different instruments, a conventional instrument and a prototype based in the "Postural Freedom" concept, during static and dynamic experiments.

- Objective G1.2. Calculate parameters to quantify the muscular activity in each muscle evaluated during the experiment. The signal will be processed in order to compare the results obtained with both instruments and to determine which one requires less effort.

- Objective G1.3. Calculate parameters in the spectral domain to quantify the muscular fatigue in each muscle evaluated during the experiment. The signal will be processed in order to compare the results obtained with both instruments and to determine which one produce less muscular fatigue.

- Objective G2.1. Define the setup for a motion capture experiment that can be used during dynamic laparoscopic simulations, without interfere in the participants movements.

- Objective G2.2. Register and evaluate the trajectories followed by each section of the dominant limb during the simulations with a conventional instrument and a prototype based on the "Postural Freedom" concept. 
- Objective G2.3. Estimate the effect of a laparoscopic instrument based in the "Postural Freedom" concept in the participants' postural health in comparison with the conventional laparoscopic instruments.

- Objective G3.1. Design of a new instrument based on "Postural Freedom" concept and develop a fully functional prototype based in the knowledge acquired with the experimentation and the feedback provided by expert users.

- Objective G3.2. Evaluate the fully functional prototype with experts and novices surgeons in order to test their satisfaction with the final solution. 




$$
\begin{array}{r}
\text { Chapter } 3 . \\
\text { Electromyography } \\
\text { evaluation }
\end{array}
$$




\section{Introduction. EMG in ergonomic studies}

\subsection{Skeletal muscle anatomy}

\subsubsection{Micro anatomy}

About $40 \%$ of the body is skeletal muscle [77] [78] and roughly another $10 \%$ is smooth and cardiac muscle [78]. The skeletal muscles are composed of hundreds to thousands of muscle fibers, plus connective tissue wrappings, blood vessels, and nerve fibers. Each muscle fiber is made up of myofibrils arranged in parallel, the myofibrils of sarcomeres arranged in series and the sarcomeres of interdigitating actin and myosin filaments (Figure 21).

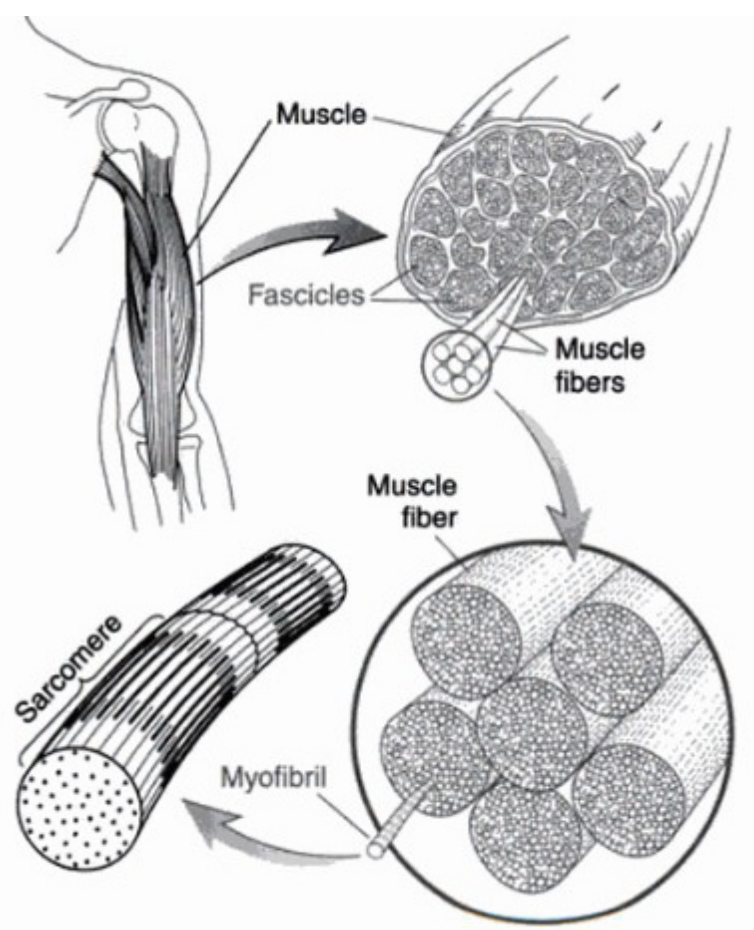

Figure 21. Muscle architecture presented by R. Lieber in 2009. Adapted from [79]. 
Each fiber extends the entire length of the muscle. Except for about 2 percent of the fibers, each fiber is usually innervated by only one nerve ending, located near the middle of the fiber [78].

The design of each muscle varies depending on the range of motion and the force exerted [77], in the most simple arrangement (fusiform), parallel fibers extend the full length of the muscle and attach to tendons at both ends (Figure 22a). Muscles producing a large force have a more complicated structure in which many short muscle fibers attach to a flat tendon that extends over a large fraction of the muscle. This arrangement (unipennate) increases the cross-sectional area and thus the contractile force of the muscle (Figure 22b). When muscle fibers fan out from both sides of the tendon, the muscle structure is referred to as bipennate (Figure 22c).

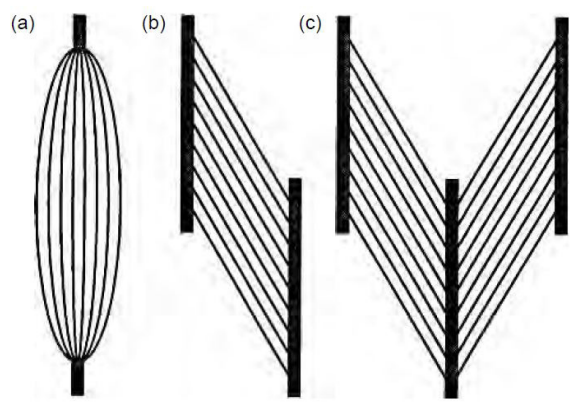

Figure 22.Schematic illustration of different types of muscles: (a) fusiform, (b) unipennate, and (c) bipennate. Adapted from [77].

\subsubsection{Macro anatomy. Muscles affected during laparoscopic procedures.}

Various methods have been used to quantify muscular effort and fatigue in laparoscopy. These studies have established a correlation among effort, fatigue and possible neuromuscular injury. However there is little published information regarding which muscle groups are at greatest risk in these procedure [75].

In 2006, D. Reyes [75] presented a review in which listed a summary of injury cases on the literature of the moment. Pain and injuries in thumb, phalanxes, shoulder and neck were reported, the duration for these injuries ranged from hours to 2 months. 
Pain from back, neck and shoulders are also common symptoms [39] and deltoids and trapezius muscles has been identify as the muscles that require more time activation despite of the task performed [80].

The scissor handle of the instruments has been consider one of the main causes of injuries in laparoscopic surgery. According to A. Park et al. $74,4 \%$ of surgeons identified the instrument design as the cause of their physical complaints [59]. The pressure of the rings over the digital nerves of the thumb and the fingers is consider a problem for many surgeons and a risk of permanent damage if laparoscopic surgeons perform procedures with instruments which do not fit their fingers [81]-[87]. The action of held the instrument in vertical position (position commonly used to introduce the instrument in the abdomen) is the main cause of tingling and paresthesia over the thenar eminence of the hand, this injuries is due to the forced flexion at the wrist joint and the feeling lasted for almost 3 weeks [85].

\subsection{Muscle performance}

\subsubsection{Physiology}

The capability to generate force can be finely regulated, with voluntary control of the rate and extent of motor unit (MU) activation. The term $\mathrm{MU}$ is referred to all the muscle fibers innervated by a single nerve, each nerve fiber innervates multiple muscle fibers depending on the type of muscle. The electrical excitation required to generate force by the skeletal muscle is initiated and regulated by the central and peripheral nervous systems [77]. A muscle contraction occurs in different steps [78]:

- The action potential generated by the CNS travels along a motor nerve to its endings on muscle fibers.

- At each ending the nerve secretes a small amount of a neurotransmitter substance called acetylcholine. The acetylcholine acts on a local area of the muscle fiber membrane to open multiple "acetylcholine-gated".

- The acetylcholine-gated channels allows large quantities of sodium ions to diffuse to the interior of the muscle fiber membrane which initiates an action potential at the membrane. 
- The action potential travels along the muscle fiber membrane in the same way that action potentials travel along nerve fiber membranes. The action potential depolarizes the muscle membrane, and much of the action potential electricity flows through the center of the muscle fiber, causing the sarcoplasmic reticulum to release large quantities of calcium ions that have been stored within this reticulum.

- The calcium ions initiate attractive forces between the actin and myosin filaments, causing them to slide alongside each other, which is the contractile process.

- After a fraction of a second, the calcium ions are pumped back into the sarcoplasmic reticulum by a $\mathrm{Ca}^{++}$membrane pump and remain stored in the reticulum until a new muscle action potential comes along; this removal of calcium ions from the myofibrils causes the muscle contraction to cease.

\subsubsection{Electrical signal associated to the skeletal muscle}

The first logical deduction of the electricity transmitted by the muscles was documented by Italian Francesco Redi in 1666. He suspected that the shock of the electric ray fish was muscular in origin and wrote, "It appeared to me as if the painful action of the electric ray was located in these two sickle-shaped bodies, or muscles, more than any other part" [88], [89].

The relationship between electricity and muscle contraction was reported by Aloysii Galvani in 1791 [90]-[92]. He depolarized the muscles of a frog's legs by touching them with metal rods. This discovery is generally acknowledged as representing the birth of neurophysiology, thereby making Galvani the father of this field which continued to expand rapidly [88]. Galvani reaffirmed his concept when he found that a muscle contraction could be caused by placing the free end of a nerve across a muscle without the intervention of metals. In 1867, G.B. Duchenne presented a work called Physiologie des mouvements [93] where he applied electrical stimulation to investigate systematically the dynamic and functions of intact skeletal muscles. This work was consider the greater contribution to the understanding of muscular function [88]. 
The electrical activity (EA) in a muscle is determined by the number of muscle fibers recruited and their frequency of excitation. However, the size of the signal is also determined by the size of the individual muscle fiber potentials, which cannot be assumed to be uniform for different muscle fiber types nor to necessarily remain constant under different experimental conditions [94]. From the study of the muscles signal can be obtained useful information that researchers can use in a wide class of applications [43], [95] such as diagnostic tool for identifying neuromuscular diseases or control signal for prosthetic devices such as prosthetic hands, arms, and lower limbs.

\subsection{EMG recording techniques}

The EMG signal is the electrical manifestation of the neuromuscular activation associated with a contracting muscle [88], [96]. The EMG technique is the technique used to measure this electrical signal associated with the activation of the muscle regardless of it is voluntary or involuntary.

As explained above, to contract a muscle the action potential generated by the CNS causes a depolarization on the muscle fiber. This depolarization generates an electromagnetic field that can be measured as a voltage [96].

To detect this voltage there is a wide variety of types of electrodes. Their use depends on the function, but the basic rule to choose, is that they must be relatively harmless for the task and must be located close enough to the muscle under study to acquire the current generated by the ionic movement [88]. The two main families of electrodes used for the study of muscle behavior are intramuscular electrodes (needle or wires introduced into the muscle tissue) or surface electrodes (sensors placed on the surface of the skin) [88], [97]. 


\subsubsection{Intramuscular EMG}

Intramuscular EMG or needle EMG is used for electro-diagnostic, the physicians with the information provided by the intramuscular EMG can confirm a clinically suspected diagnosis, exclude other potential diseases, identify unrecognized, localize abnormality or lesion within a specific region of the peripheral nervous system, define the severity of a disease, define the pathophysiologic mechanism of a disease and define the evolution, stage and prognosis of a disease [98].

To use the intramuscular EMG technique is necessary to puncture the muscle with a needle. The muscle is localized by palpation and then, the skin is tighted to decrease the pain that occurs during the insertion of the needle [98]. The intramuscular EMG is an invasive technique and it can cause pain and discomfort to the patient. Movement of the needle through the muscle is the main generator of discomfort. Despite this technique is a safe procedure, potential complications related to needle insertion and movement through a muscle may occur in patients with anticoagulation or bleeding disorders, patients with obesity and patients with low pain tolerance [98].

\subsubsection{Surface EMG}

Surface electrodes represent a non-invasive approach to acquire this signal. These electrodes can be constructed as either passive or active. In the passive configuration, the electrode consists of a detection surface that senses the current on the skin through its skin-electrode interface. In 1912 was the first time that was reported the use of this electrodes specifically for the purpose of detecting EMG signals from a human muscle [99] and the design of passive surface electrodes does not change too much and conceptually it today works similarly [88]. In the active configuration, the input impedance of the electrodes is greatly increased, rendering it less sensitive to the impedance (and therefore quality) of the electrode-skin interface [88]. The active surface electrodes have been developed to eliminate the need for skin preparation and conducting medium. They are often referred to as "dry" electrodes [88]. 
The use of sEMG to study dynamic movements began in 1940 but until 1960s the sEMG was not used for the treatment of specific disorders. The work about spectral analysis and fatigue presented by DeLuca and his colleagues of the Neuromuscular Research Institute in Boston shed light on the physiology of muscle and methods of measuring it [100].

In biomechanics, the SEMG has been used in a wide range of studies, but there is three principal applications for this technology [101]:

- Muscle activation indicator: As an indicator of the initiation of muscle activity, the signal can provide the timing sequence of one or more muscles performing a task.

- Indicator of the force produced by a muscle: To provide information about the force contribution of individual muscles as well as groups of muscles. The ability to determine with non-invasive methods the force contribution of individual muscles provides an enormous advantage, particularly when biomechanical models are developed to describe the workings of a segment of the musculoskeletal system

- Muscular fatigue index: As fatigue index is really attractive because it has the potential to predict the beginning of the muscular fatigue by means of time-dependent changes.

For this study, the last two applications (indicator of force produced by the muscle and muscular fatigue index) are relevant, nevertheless the signal analysis has to be carefully performed.

\subsection{Surface EMG analysis}

As previously explained, sEMG supposes an easy access to the physiological processes that allow us to generate muscle's force. This technology has the potential to predict the onset of contractile fatigue by means of time-dependent changes, even before that the force modification occurs [101]. 
The first study reporting this phenomenon was presented in 1912 by H. Piper [99], he found a decreased in the frequency parameters during fatigue. Later, in 1923, Cobb and Forbes [102], found that not only the frequency parameters decrease but also the amplitude of the waves increases during muscular fatigue, indicating that action-currents of greater voltage are produced in the fatigued muscle. After this discovery, a lot of researchers validated these results, founding an increase in the signal amplitude during fatigue [22], [103]-[107] and a decrease of frequency parameters [22], [105], [108]-[111] in a variety of muscles throughout the human body. These two phenomena are related and, Lindström[112] in 1970 and De Luca [113] in 1979, explained this relationship (Figure 23).
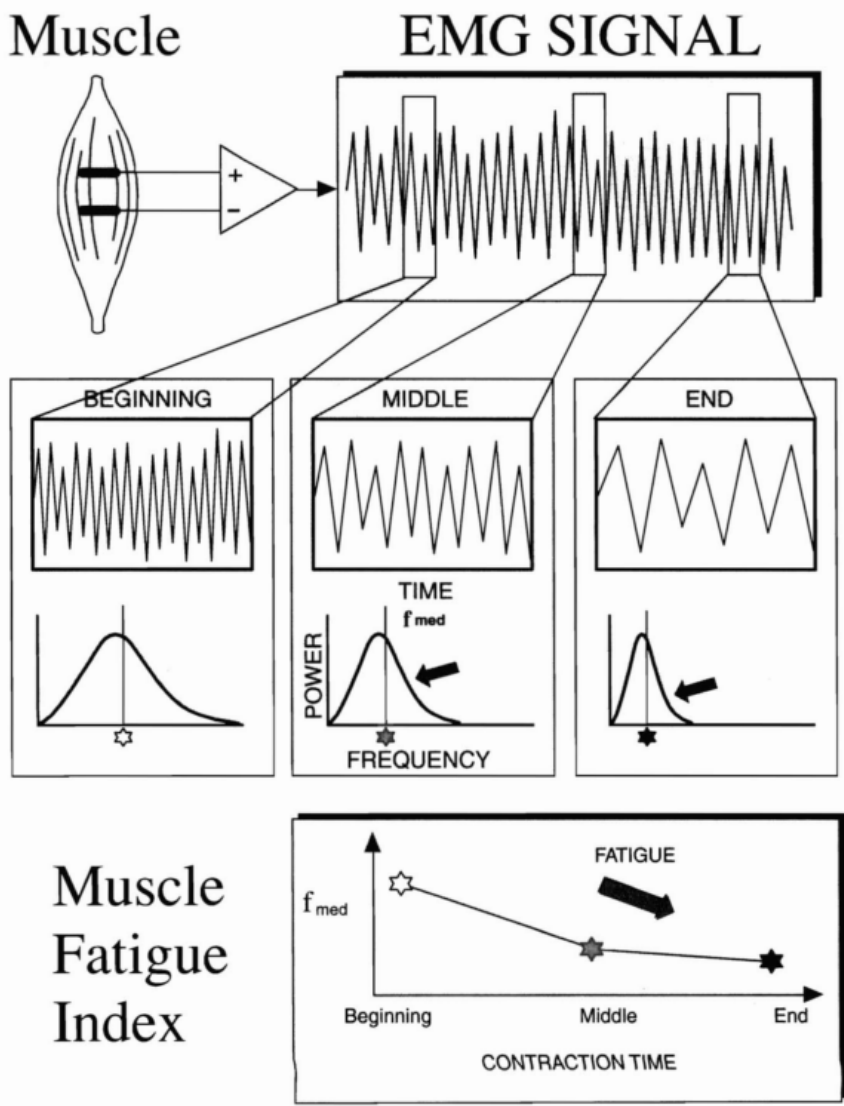

Figure 23. Diagrammatic explanation of the spectral modification that occurs in the EMG signal during sustained contractions. The muscle fatigue index is represented by the Fmed of the spectrum. Adapted from [101]. 
They noted that during a sustained contraction the low-frequency components increase and, hence, more energy will be transmitted through the low-pass filtering effect of the body tissue. Therefore they also noted that the magnitude of the two related phenomena is dependent of many factors, such as force level of contraction, time into the contraction, the type of electrode used to obtain the signal, the thickness of the subcutaneous tissue, and the particular muscle investigated.

Also is commonly observed that the spectral shift is most dramatic near the beginning of a sustained contraction (Figure 24), whereas the amplitude of the myoelectric signal shows a more pronounced increase near the end of a sustained contraction.

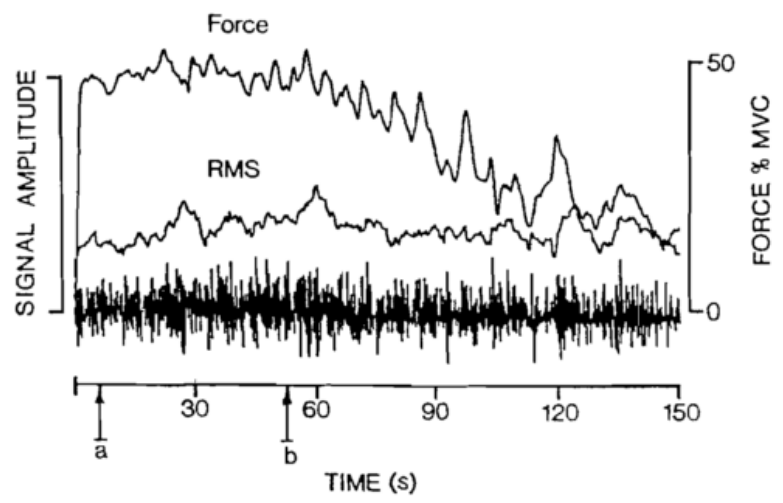

Figure 24. Myoelectric signal amplitude and force during an attempted constantforce contraction in the first dorsal inter-osseous muscle. Adapted from [22].

\subsubsection{Analysis to measure the muscular effort. Amplitude parameter}

In EMG, the term amplitude can be taken as a global measure of MU activity during the muscle action being performed and it defines the fluctuation or displacement of the muscular activity from its mean value. As explained above, in 1923, an study of Coob and Forbes [102] founded an increase in the amplitude of the waves during muscular fatigue. The standard amplitude parameters commonly used in classical signal processing applications are the ARV (Average rectified Value) and RMS (Root Mean Square) [114]. The ARV value is the area between the rectified signal and the time axis (signal integral) computed during a time interval and divided by the time, therefore providing a mean voltage value. RMS is obtained by dividing the energy of the signal during a time interval $T$ of duration by the time $T$, thus providing a mean power value whose square root is the RMS value. 


$$
v_{r m s}(t)=\sqrt{\frac{1}{T} \int_{-\frac{T}{2}}^{\frac{T}{2}} v^{2}(t) d t}
$$

This parameter, in combination with the frequency parameter, is also used to estimate the localized muscular fatigue [115].

\subsubsection{Analysis to measure the muscular fatigue. Mean and median frequency parameter}

Frequency is a parameter that determines how often the sinusoidal signal goes through a cycle. It describes the number of times that the signal is repeated per second.

To transform the SEMG signal in the time-domain to the frequency-domain is employed a technique based in Fourier transformation to provide the power spectrum or the Power Spectral Density (PSD). The PSD is the frequency response of a random or periodic signal. It tells us where the average power is distributed as a function of frequency. According to Fourier transformation, any physical signal (for example EMG) can be decomposed into a number of discrete frequencies, or a spectrum of frequencies over a continuous range (Figure 25). The statistical average of a certain signal or sort of signal as analyzed in terms of its frequency content is called spectrum.
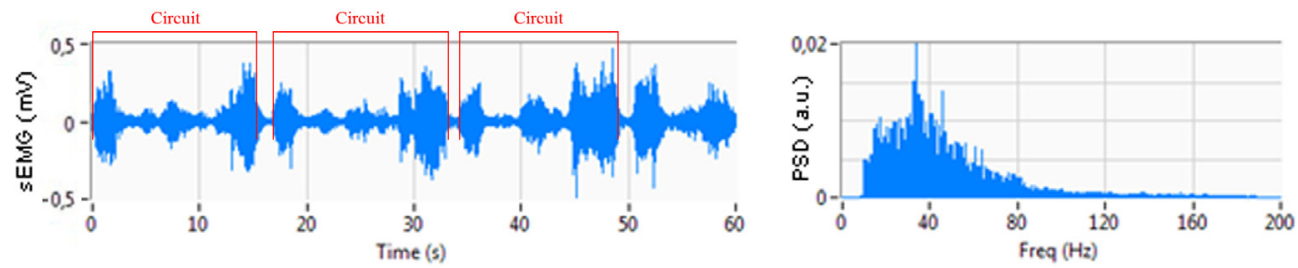

Figure 25. EMG signal and PSD obtained from one of the experiments with the conventional instrument. 
Frequency domain features show the better performance than other domain features in case of the assessing muscle fatigue [43]. Mean frequency (Fm) and Median frequency (Fmed) are the most commonly used frequency features, and are commonly used to assess muscle fatigue by means of sEMG.

The introduction of $\mathrm{Fm}$ in the assess of muscular fatigue was proposed by Linstrom and Magnusson in 1977 [116] and the use of Fmed by Stulen and DeLuca in 1981 [117].

Fm is an average frequency which is calculated as the sum of product of the EMG power spectrum and the frequency divided by the total sum of the power spectrum. The definition of $\mathrm{Fm}$ is given by the equation below, where $f_{j}$ is the frequency value of $E M G$ spectrum at the frequency bin $j, P_{j}$ is the $E M G$ power spectrum at the frequency bin $\mathrm{j}$, and $\mathrm{M}$ is the length of frequency bin [43].

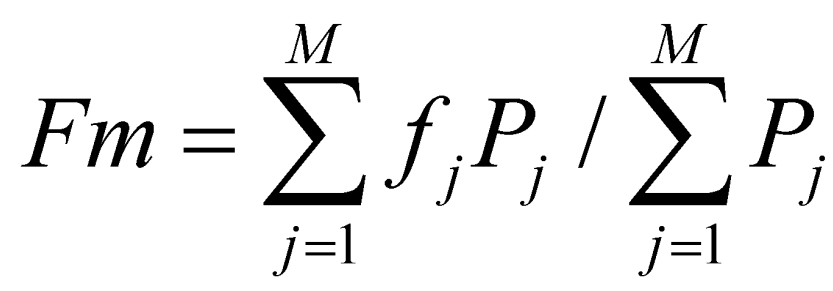

Fmed is a frequency at which the EMG power spectrum is divided into two regions with equal amplitude. Fmed is also defined as frequency from the half of the total power. The equation for the Fmed is presented below:

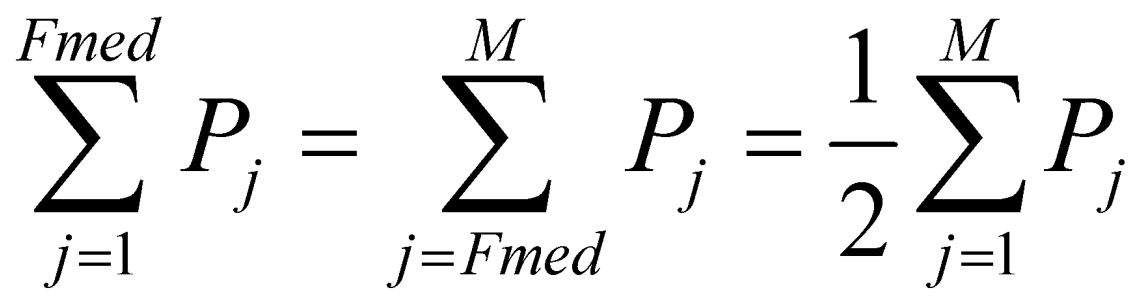

The behavior of Fm and Fmed is similar. However the performance of both frequency features is quite different [43]. Fmed has been shown to be less sensitive to added white noise and in most cases more sensitive to the biochemical and physiological processes that occur within the muscles during sustained contractions [22], [114]. In contrast, Fm may generally be estimated with a lower relative error [114]. 
Theoretically, both Fmed and Fm are equally sensitive to spectral modifications, but in practice, Fmed has been found to be more sensitive to modifications that occur in the myoelectric signal during sustained voluntary contractions [50], [114], more affected by muscle fatigue [43], [117], and less affected by random noise, particularly in the case of noise located in the high frequency band of EMG power spectrum [43] being this parameter more appropriate to evaluate small signal-noise ratios [118].

\subsection{Muscular fatigue index}

In industrial ergonomics it is well recognized that static as well as dynamic postural stress can lead to fatigue and disability [7], [119], [120]. The next headings explore the different approaches used over time to evaluate the muscular fatigue in a variety of static and dynamic postures.

\subsubsection{Failure point approach}

Traditionally, physiologists used to employ the force output of a muscle as the index of muscular fatigue. In particular, the point at which the desired force output may no longer be maintained and contractile fatigue becomes observable [22]. The main problem of this approach is that the use of an indicator as the failure point (Figure 26) means that the fatigue is detected only after this point occurs, a notion inconsistent with the concept of fatigue accepted by engineers and physical scientists [101], who have consider the concept of fatigue as a time-dependent process.

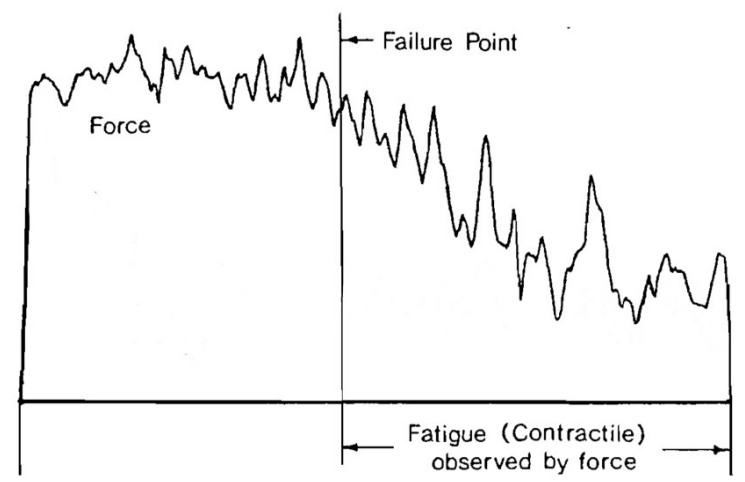

Figure 26. Contractile fatigue. The failure point denotes the time when the force output was no longer maintained at the desired average value. The time duration of the contraction was 150 seconds. Adapted from [22]. 
Other confounding factors derived from this approach [101]:

- During a voluntary contraction, the force of an individual muscle is not often directly accessible, and the monitored torque may not faithfully represent the force of the muscle of interest.

- In submaximal contractions is possible to maintain the torque acceptable constant but there are time-dependent physiological and biochemical processes that microscopically alter the means for generating force during a sustained contraction as recruitment of MUs, decrease in the firing rated of most MUs and increase the amplitude of force twitches of MUs.

- The failure point is a function of both physiological and psychological factors, and it is difficult to know accurately the causal relationship of each to the failure point.

\subsubsection{Spectral modifications approach}

De Luca identified an alternative approach [22]. This approach use the spectral modification property of the EMG signal during a sustained contraction. During these sustained contractions, the spectral modifications provide fatigue indices that describe the time course of the fatigue-related physiological and biochemical processes [101]. The access to biochemical and physiological data within the muscle or the nervous system can reveal time-dependent changes indicative of a fatigue process, even though the externally observable mechanical performance would not be altered until the failure point (Figure 27).

The spectral modification approach presented by De Luca provides at least two advantages over the traditional contractile fatigue approach:

- Contractile force can only be conveniently measured by monitoring the torque about a joint to which more than one muscle can contribute. In contrast, the EMG signal can be detected from individual muscles; thus, the spectral variable fatigue index can be used to describe the performance of individual muscles. 


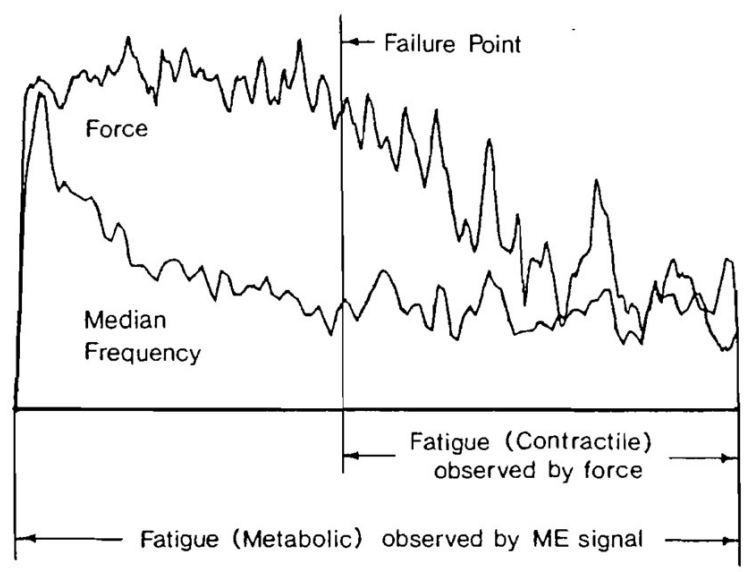

Figure 27. Distinction between contractile fatigue and metabolic fatigue. The metabolic fatigue is observed at the beginning of the contraction while the failure point appears once the contraction cannot be maintained anymore. The time duration of the contraction was 150 seconds. Adapted from [22].

- Spectral modification progresses continuously from the onset of contraction, thus indicating the rate of the fatigue process early in the contraction. Contractile fatigue, as currently measured, requires the subject to expend considerable effort before fatigue is measurable.

The mechanisms that cause EMG spectral modification and those that generate the force both experience changes during the progression of fatigue. That means that a relationship exists between them [101].

The spectral modification may be monitored and quantified by tracking some characteristic indicators of the frequency spectrum, such as the median, mean, or mode frequency of the spectrum, or alternatively by calculating a ratio of lowfrequency to high-frequency bandwidths, or by integrating the area corresponding to the decrease of the Fmed [101]. But, as explained above in the section "Analysis to measure the muscular fatigue. Fm and Fmed parameters" Fmed is consider as the more reliable of these parameters [22], [101], [114]. 


\subsubsection{JASA approach}

Despite amplitude and frequency parameters are widely used to determine fatigue during muscular contractions [22], [103]-[111] , they have the disadvantage that they not only change under the influence of muscular fatigue, they also depend on the contraction level of the particular muscle. For this reason, A. Luttmann et al (1996) [121] present the principles of a method called JASA (Joint Analysis of the Spectral and Amplitudes). This method presents four different scenarios (Figure 28) that could occur during muscular contractions [121]-[123]:

- The first scenario presents a decrease of the signal amplitude and an increase of the Fmed indicating a recovery of the muscle.

- The second scenario identify a decrease of the amplitude and also a decrease of the Fmed, this indicates a decrease in the force performed.

- The third an increase of the amplitude and also an increase of the Fmed indicates that the force is increasing.

- And the fourth scenario presents an increase of the amplitude and a decrease of the Fmed, which indicates fatigue.

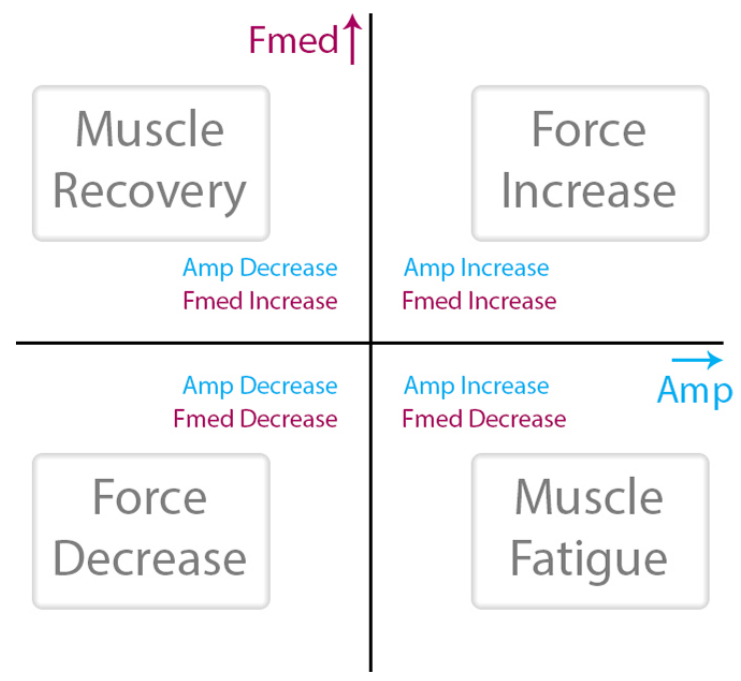

Figure 28. JASA scenarios. 
Luttmann et al. on this study [121] talks about EA and Fmed. But the term EA was modified by EMG amplitude, the reason to this change is that the EMG signal is affected by noise from the equipment, the surrounding environment, and even the nature of the signal itself, for this reason, the biomedical researchers usually uses the term amplitude rather than EA, muscle activation or muscle effort in the context of EMG during muscle fatigue [22], [50], [77], [101], [116], [122]-[125]

\subsection{Other methods to detect muscular fatigue}

Although EMG has been chosen as the most suitable clinical research tool to acquire muscle fatigue, there are several other sensors and signal acquisition methods which have been used in studies on muscle fatigue [126] and they can be useful to understand the context of this research work:

- Mechanomyography (MMG), considered as the mechanical equivalent to sEMG [126], [127], it detects the mechanical signal from the surface of the contracting muscle and record the vibrations in the muscle [128]. It has been found that MMG is capable of detecting individual muscle actions and can distinguish between central and peripheral fatigue [126], [129], [130]. This signals have been recorded using different sensors as hydrophones, acoustics, condenser microphones, piezoelectric contact sensors, goniometer, accelerometer, or laser distance sensors [126], [128], [131]-[133]. In studies of localized muscle fatigue in dynamic contractions, the MMG amplitude has been investigated for both concentric and eccentric muscle contractions [134]-[136].

- Near-Infrared Spectroscopy (NIRS) is a non-invasive method that uses the near infrared part of the electromagnetic spectrum to measure the absorption properties of blood hemoglobin [126], [137]. During exercise, the intramuscular pressure restricts the blood flow, causing a significant decrease in oxygenation and blood volume. On the other hand, muscle contraction demands more oxygen delivery to the area, which increase blood flow. 
Changes in blood flow near the skin can be detected using NIRS [126]. NIRS can be used as a measure of oxygenation changes in a fatiguing muscle [138], revealing information about local blood circulation, blood volume and changes in the oxygenated hemoglobin in the contracting muscle [126].

- Sonomyography (SMG) is a system that uses ultrasound to describe the structural and morphological changes of skeletal muscles and it has been used to detect changes in the muscle during muscular fatigue [139], [140]. In 2007, an interested results was founded by SMG, in biceps brachial muscle the thickness of the muscle increased first rapidly then gradually during the process of fatigue. The SMG can be used as an additional method together with $\mathrm{SEMG}$, providing more information about the fatiguing muscle, for example, the torque fluctuation due to the failure to maintain the required contraction level [126], [140].

\subsection{Introduction to the experiments}

As explained in the introduction, the MAS procedures require more effort, concentration and mental stress than open procedures [60], and forces the surgeons to adopt non-neutral postures with phalanges, hands, wrists arms [35], [60], [70], fatiguing the surgeons body and creating potential for errors that may harm the patient during the operation [141].

The poor ergonomic postures accelerate the muscle fatigue and pain process because, outside the neutral range, muscles require more energy to generate the same contractile force than in neutral positions [142]. This is the reason why is commonly attributed higher muscular fatigue and levels of discomfort to MIS procedures in comparison to the conventional techniques [35], [143].

During this study, the implementation of an articulated element that allows PF with the aim to reduce the amount of non-neutral postures during the MAS procedures was evaluated. The goal of the experiment is to identify the effect that the PF prototype has on the musculature in comparison to the conventional pistol grip handle. The symptoms evaluated during this experiment were the muscular activity 
levels and the localized muscular fatigue. The muscles evaluated were in charge of the main movements performed in laparoscopic procedures: Trapezius, Deltoids, Biceps and Flexor Carpi Radials. These muscles were chosen after observations of procedures (in situ and online) and after performance of practices in the simulation area.

Trapezius muscle is one of the major muscles of the back and is responsible for moving, rotating and stabilizing the scapula and extending the head at the neck. It is a superficial, large, fan like muscle found on the back. It arises from the occipital bones, occipital protuberance and nuchal lines, as well as the spinous processes of C7 through T12. It inserts onto the spine of the scapula, acromion, and lateral third of the clavicle. The muscle can be divided into three sets of fibers: upper, middle, and lower. During this experiment, the upper fibers of trapezium has been evaluated. These fibers are the responsible to elevate and upwardly rotate the scapula and extend the neck (Figure 29). The middle fibers adduct (retract) the scapula. The lower fibers depress and help upper fibers upwardly rotate the scapula.
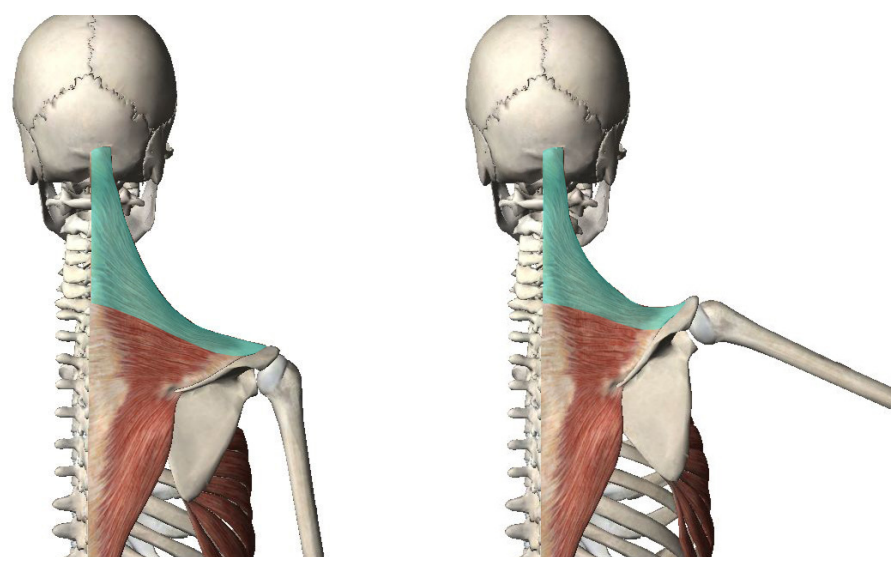

Figure 29. Trapezius muscle. Colored section identifies the superior fibers.

Figure obtained from Visible Body Software.

Deltoid muscle is named due to its Greek delta letter shape (triangular) appearance. It is a powerful superficial muscle of the shoulder. Like the trapezius, this muscle can be divided into three sets of fibers: anterior, lateral, and posterior. Due to this arrangement the deltoid has a large area of origin: from the acromion, lateral superior portion of the clavicle, and lateral third of the scapular spine. It inserts onto the deltoid tuberosity, which is a roughened elevated patch found on the lateral surface of the humerus. As a result it acts as a flexor, extensor, and abductor of the shoulder. It also assists in medial (anterior fibers) and lateral rotation (posterior fibers). The anterior fibers are located in the frontal part of this muscle (Figure 30). 

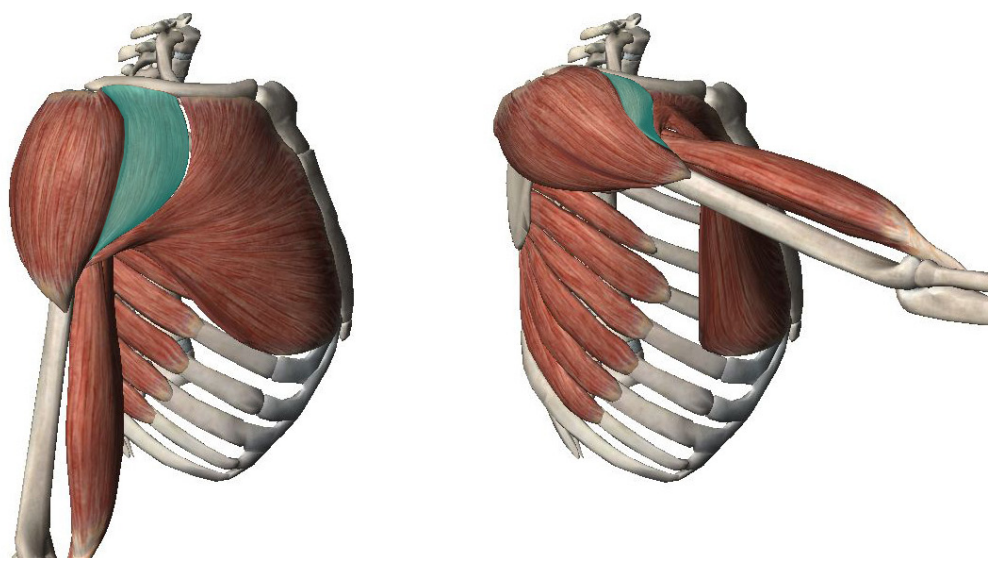

Figure 30. Deltoids muscle. Colored section identifies the anterior fibers.

Figure obtained from Visible Body Software.

The biceps brachial muscle is a superficial muscle that forms the bulk of the anterior compartment of the arm (Figure 31). It has a long head and a short head. The long head arises from the supraglenoid tubercle of the scapula and passes through the intertubercular sulcus in its own synovial sheath. The short head arises from the coracoid process and both heads unite.
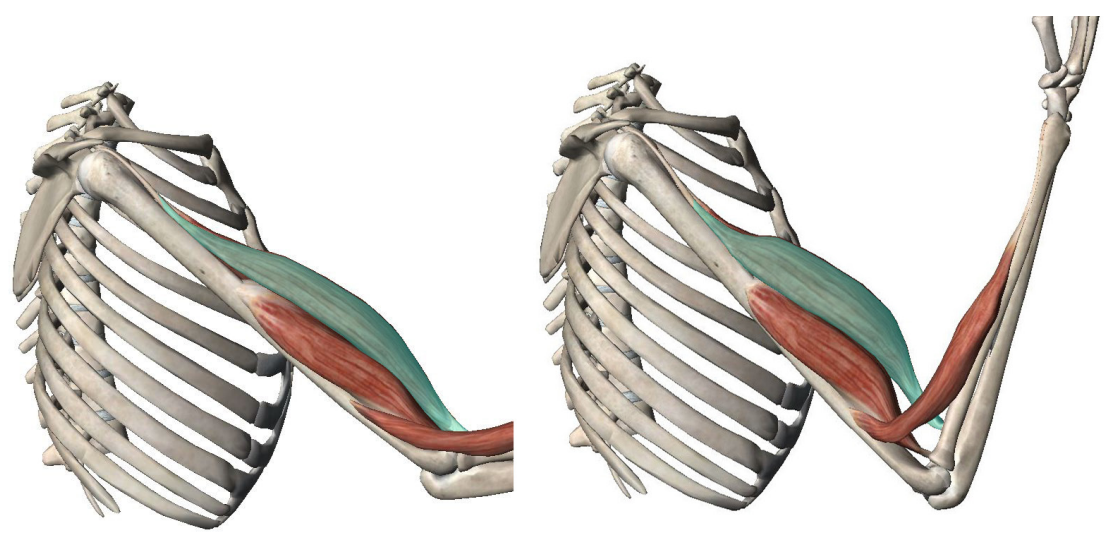

Figure 31. Biceps muscle. Colored section identifies the muscle. Figure obtained from Visible Body Software.

The muscle then descends inferiorly to insert into the radial tuberosity of the radius as well as help create the bicipital aponeurosis, an expansion that inserts into the deep fascia of the forearm and onto the ulna. The muscle acts primarily as a supinator of the forearm, as well as a flexor of the elbow. Its supinating effect are maximal when the elbow is flexed. It is innervated by the musculocutaneous nerve. 
The Flexor Carpi Radials is a thin muscle located on the anterior part of the forearm (Figure 32). It arises in the humerus epicondyle, close to the wrist area, travels on the outside of the flexor digitorum superficialis and inserts at the base of the index finger. The innervation of this muscle is provided by the median nerve and it receives its blood supply through the radial artery. This muscle performs the function of providing flexion of the wrist and assists in abduction of the hand and wrist.
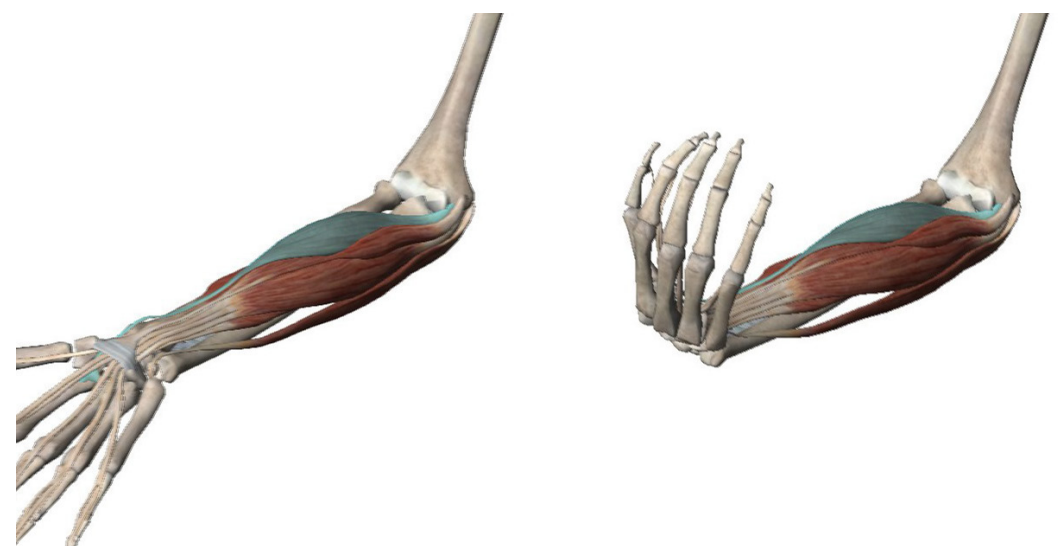

Figure 32. Flexor carpi radials. Colored section identifies the muscle.

Figure obtained from Visible Body Software

\subsection{Refining the experiment}

Because this kind of experimentation requires the test of the system several times until the right configuration is presented. The next paragraphs present a brief explanation of the path taken to finally conclude that the static experiment was the most appropriate, for the evaluation we were trying to perform.

At the beginning, a dynamic study called "Curve" (Figure 33) created by Mattern et al. in 2004 [144] was used, this test is commonly used to test the precision of new laparoscopic instrumentation.

Three red dots were added as control points indicating some extreme positions which the surgeons could achieve during the laparoscopy, the position of these three points were discussed with a group of surgeons in "La Fe" Hospital. The objective of these points was to obtain values from awkward surgical postures. 


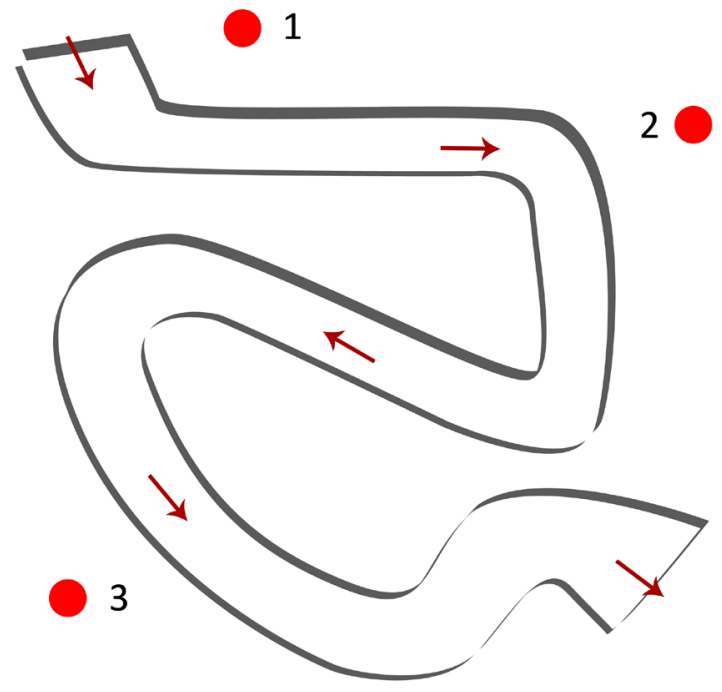

Figure 33. Curve test adapted from Mattern et al. [144]. The red dots were included to record some extreme positions, arrows indicate the direction of the test.

During this experiment, participants started from a rest position, with arms at sides of the body, then they had to introduce the instrument in the box-trainer, follow the circuit, touch the three points, and take out the instrument recovering the rest position. This cycle was repeated during 16 minutes, with a rest minute at the beginning and at the end of the experiment.

Once the test was performed with the first instrument, the participants had a recovery time of 30 minutes before the instrument exchange. The participants received the instruments randomly. Both instruments had ink based tips mounted to enable users to mark the trajectory of their motion on the template. Both lines of the circuit are separated $2 \mathrm{~cm}$ to each other.

The raw signals obtained with muscles trapezius, deltoids, biceps and flexor carpi radials after the experiment are presented in Figure 34. Despite the raw signal appear to be confuse when is presented complete, looking by minutes some patterns were founded (Figure 35). 


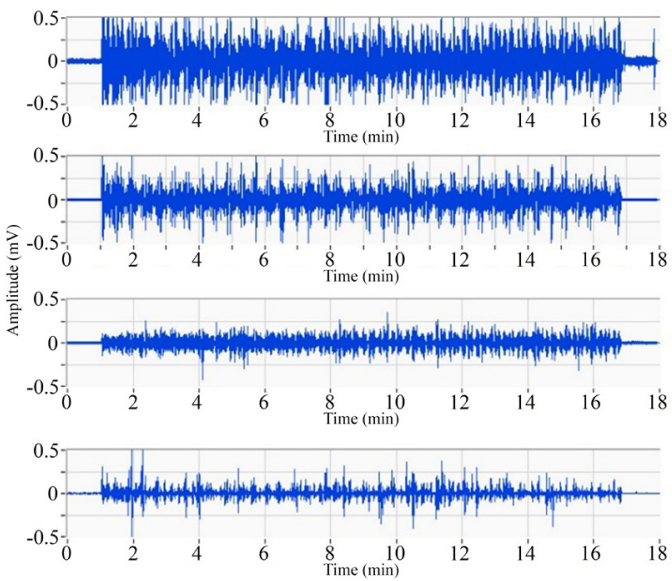

Figure 34. Raw signal obtained on the dynamic experiment with the control instrument.

The signals belongs to muscles trapezius, deltoids, biceps, and flexor carpi radials in descending order. Values in millivolts $(\mathrm{mV})$.

Evaluating the raw signal was founded that almost all the muscles presented similar peaks at entrance and exit position identifying the beginning and end of each circuit that is because the movement required to introduce and extract the instrument from the box-trainer requires great muscle effort, also the shape of the signal on the circuit present some similarities, being the parts of the template facing the participants which increased the signal, mainly in the trapezius and deltoids which are in charge to stretch forward the arm.
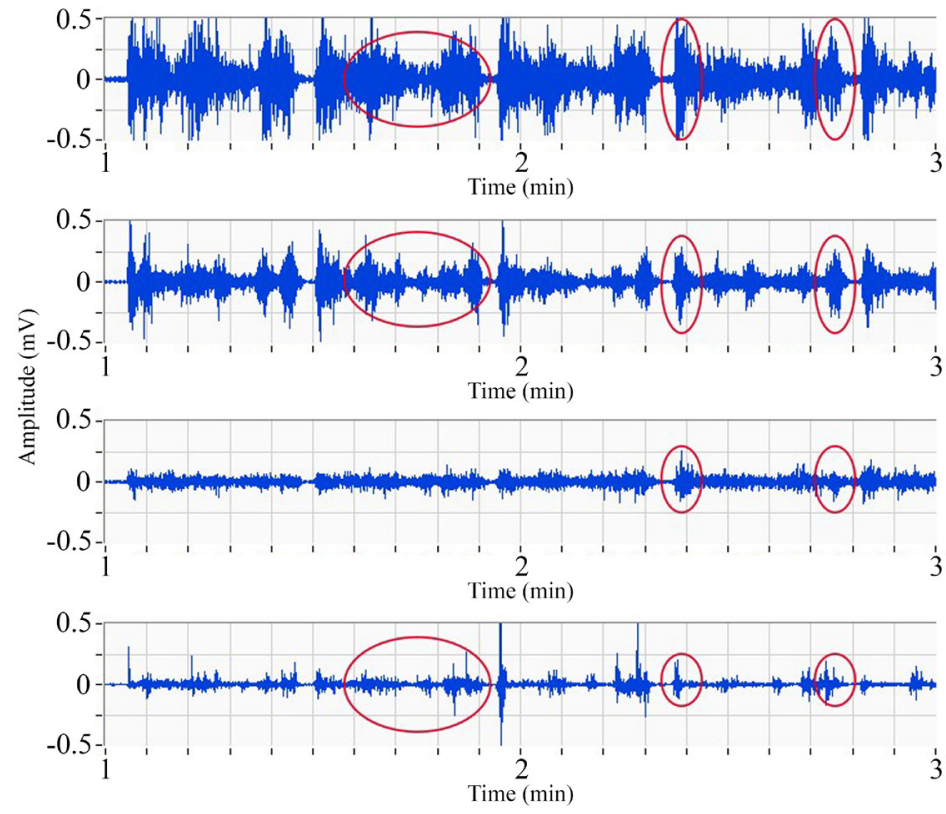

Figure 35. Raw signals of 2 minutes of the dynamic experiment with the control instrument. Red circles identify patterns founded in the signal.

The signals belongs to muscles trapezius, deltoids, biceps, and flexor carpi radials in descending order. Values in millivolts $(\mathrm{mV})$. 
This first experiment was useful in order to corroborate the benefits that the new concept of instrument has during the laparoscopic procedure, the raw signal already presented the reduction indicated in the literature (Figure 36). Muscles deltoids and trapezius seemed to be the more benefited by the new instrument.

Control
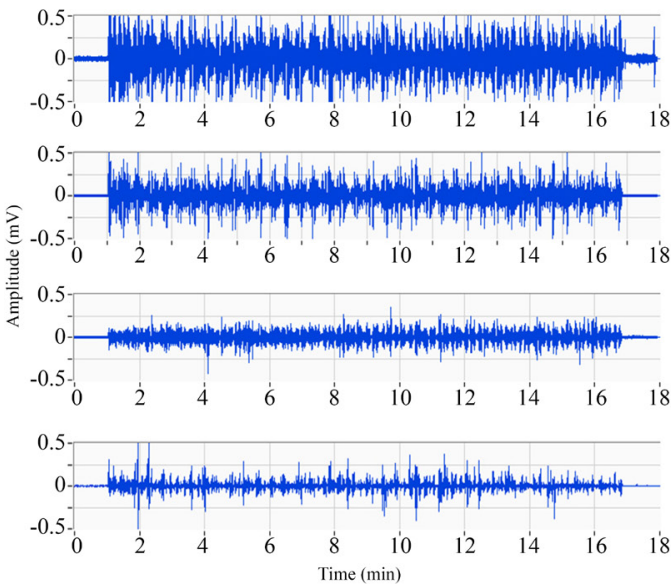

PF prototype
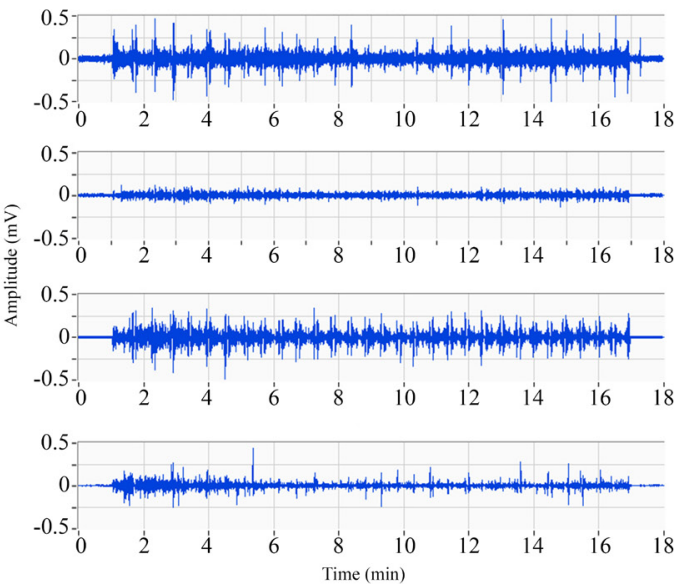

Figure 36. Raw signal generated by the dynamic experiment with both instrument. Left signals correspond to the control instrument and right signal to the prototype instrument.

The signals belongs to muscles trapezius, deltoids, biceps, and flexor carpi radials in descending order. Values in millivolts (mV).

The main problem founded after thorough observation and evaluation of this experiment was that the noise generated by the wires movement, created signals peaks that made difficult to obtain accurate results or to clean the signal. Also was founded that during laparoscopic surgery, more than $70 \%$ of intraoperative work postures are substantially static [119].

Finally, a static experiment was used in order to evaluate as accurate as possible the positions reached during the procedure. For the static experiment the participants followed a $360^{\circ}$ template (Figure 37), similar to the previously used by other authors to compare laparoscopic instruments [37], [145].

The raw signal generated by the experiment is presented in Figure 38. The 5 second of rest between targets let us identify and organize the activity values of each target, in this figure the rest positions between targets is marked with a red vertical line. 

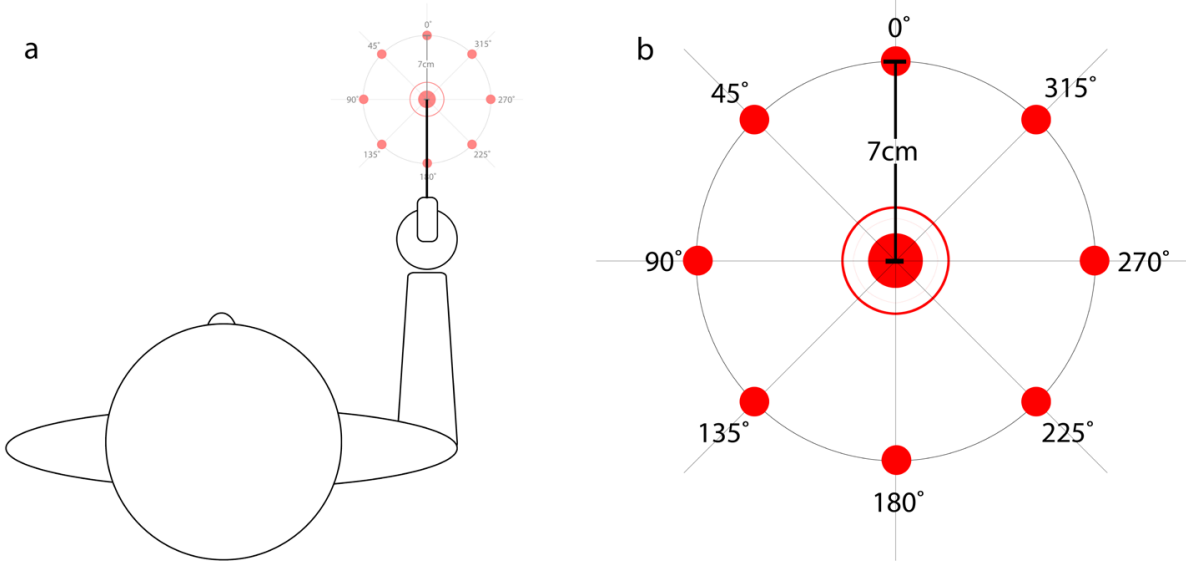

Figure 37. Left picture indicate the participant location in front of the box trainer simulator.

The shoulder is aligned with the target $0^{\circ}$. The trocar entrance is located above of the center of the template. The right picture is the $360^{\circ}$ template used during the experiment.

The participant holds the tip of the instrument at each target for 55 seconds.

In the raw signal of the static experiment (Figure 38 ) are easily traceable the different targets, the experiment begun with a rest position, this position was useful to identify the environment noise acquired by the signal. Once the noise of the signal was not significant, the participants begun the experiment.

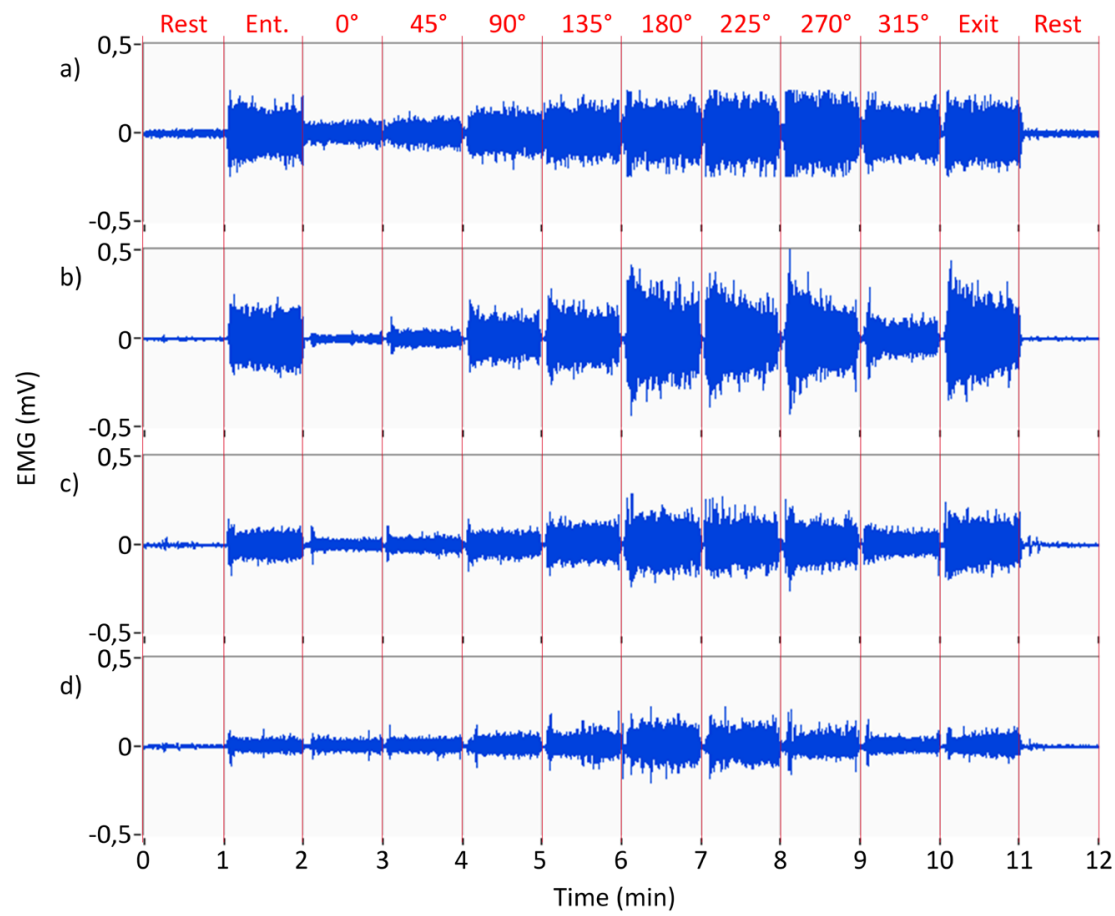

Figure 38. Raw signal of one participant during the experiment with control instrument.

Each minute represents a target. Initial and final minute are rest positions. The muscles a) trapezius, b) deltoids, c) biceps and d) flexor carpi radials are presented. 


\section{Materials and methods}

\subsection{Participants}

Seventeen participants without previous experience in laparoscopic surgery were evaluated. Both women and men of all ages and heights were included in the study without discrimination. Participants did not have any muscular pathology or numbness that caused difficulties with the movement of the upper limbs. Every participant performed the test with the dominant arm.

\subsection{Instruments}

The control instrument used to the comparison on this study is the AdTec ${ }^{\circledR}$ single use (B.Braun, Germany). This instrument presents the basic pistol grip configuration for the handle. Between the handle and the shaft of the PF prototype, a ball socket articulation of $5 \mathrm{~cm}$ diameter was added. This articulation was manufactured by 3D printing (Zortrax M200, Zortrax, Poland) and provided a constantly variable handleto-shaft angle that covers $120^{\circ}$ (Figure 39 ), this instrument do not have a blockage system.

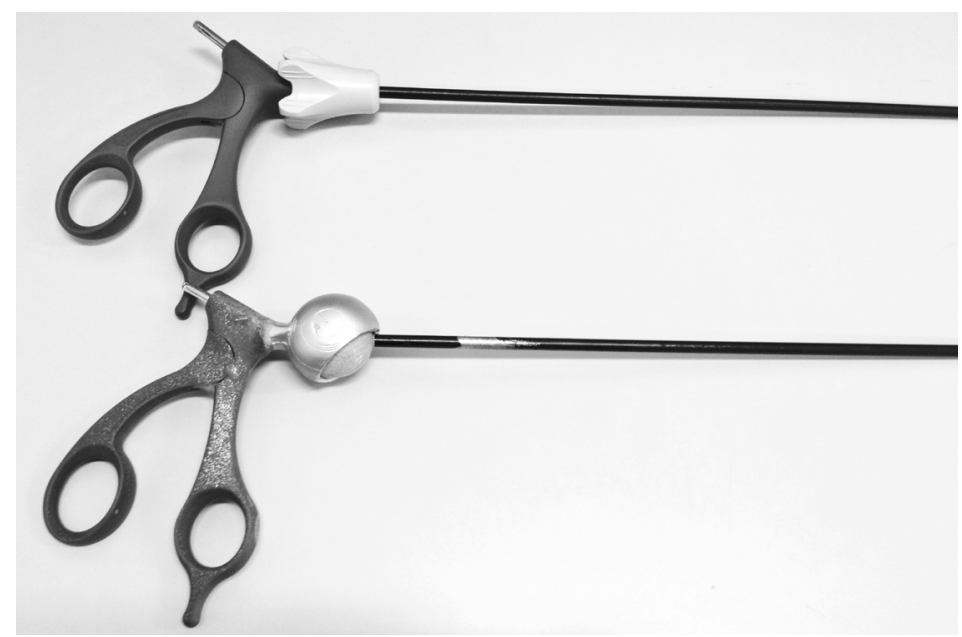

Figure 39. Both instrument used to compare the effectiveness of PF in hand-held instruments. Superior instrument is a conventional pistol-grip handle. Inferior instrument is the PF prototype. 
A $5 \mathrm{~mm}$ trocar (Endopath $\mathrm{XCEL}^{\circledR}$ bladeless trocar, Ethicon, USA) was used as port of entry in a hand-made box-trainer with the same sizes $(50 \times 30 \times 22)$ than the conventional box-trainers used in the hospital la Fe (Figure 40).

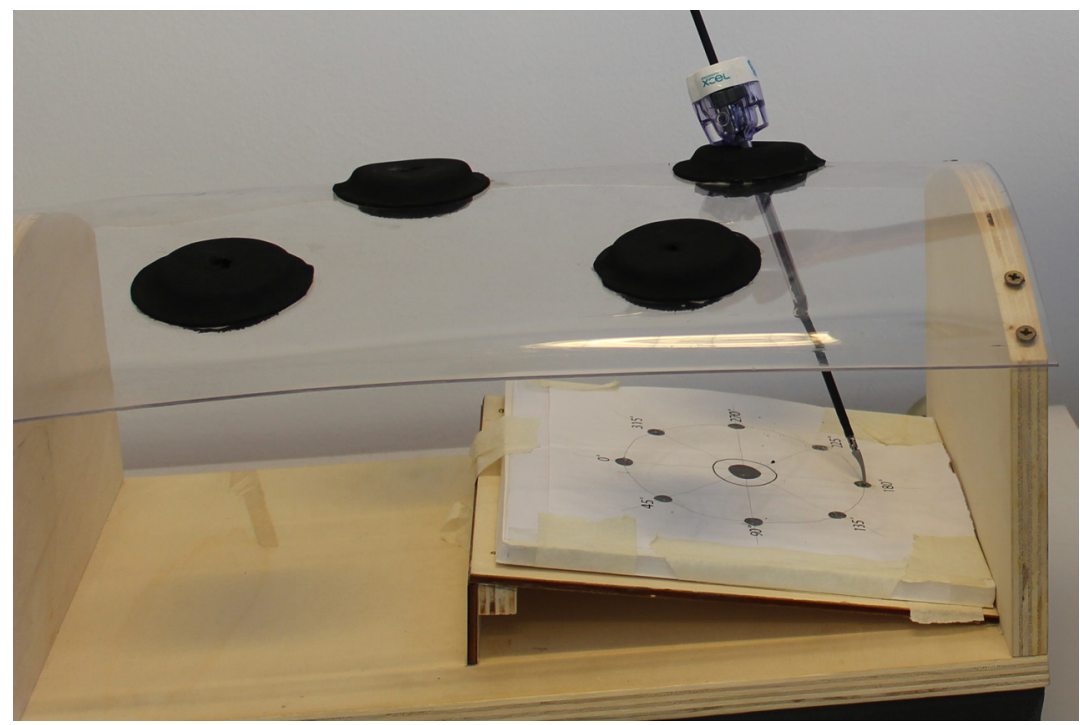

Figure 40. Box-trainer and trocar used in the sEMG experiment.

\subsection{Protocol}

During this experiment the EMG activity in different muscles was recorded and results with the control instrument were compared to results with the PF prototype. Prior to data collection, the experiment was explained to the participants to avoid stops once it was working.

The participants maintained the position of the body in a static posture for 55 seconds, reaching each target of the 360 degrees template (Figure 41) and the Entrance-Exit targets. Entrance and Exit targets were the same posture at the beginning and at the end of the test, in this target, the participant maintain the tip of the instrument inside the cannula of the trocar. Some variations of this degrees test was used for other authors with the aim to compare different instruments [37], [145]. 
Five seconds of rest is needed in order to change between targets. During this inter-target rest a member of the team moved the instrument to the next target. Two 60 seconds rest positions were recorded before and after the experiment to characterize the signal-noise ratio.

Participants received the instruments in a random order and they rested (10 min) between each experiment. The experiments were performed inside a Faraday cage in order to reduce power electric interferences. The participants received this exact explanation before the experiment and they were guided during the whole test process.

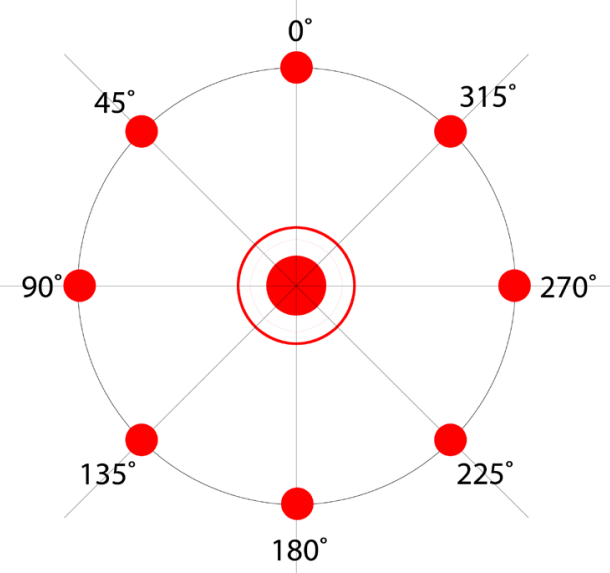

Figure 41. 360 Degrees template. During the experiment, this template is located centered below of the trocar incision to force the subject to reach positions with different level of effort during the experiment.

\subsection{Data acquisition}

The SEMG was used in order to analyze muscular activity in each muscle and the localized muscular fatigue produced after the whole experiment. The muscles evaluated during this experiment were trapezius (superior fibers), deltoids (anterior fibers), biceps (long head) and flexor carpi radials. 
Two bipolar disposable electrodes $\mathrm{Ag}-\mathrm{AgCl}$ were located in each muscle to acquire the signal. The electrodes were located following the SENIAM (surface EMG for non-invasive assessment of muscles) recommendations [146] and were covered by elastic bands to avoid artefacts caused by the movement of the wires [88]. The reference electrode was a stainless steel plate placed on the wrist of the dominant arm.

Also a reference electrode (Figure 42) is necessary for providing a common reference to the differential input of the preamplifier. The reference electrode was placed on the wrist of the dominant arm because it needs to be on electrically neutral tissue [147].

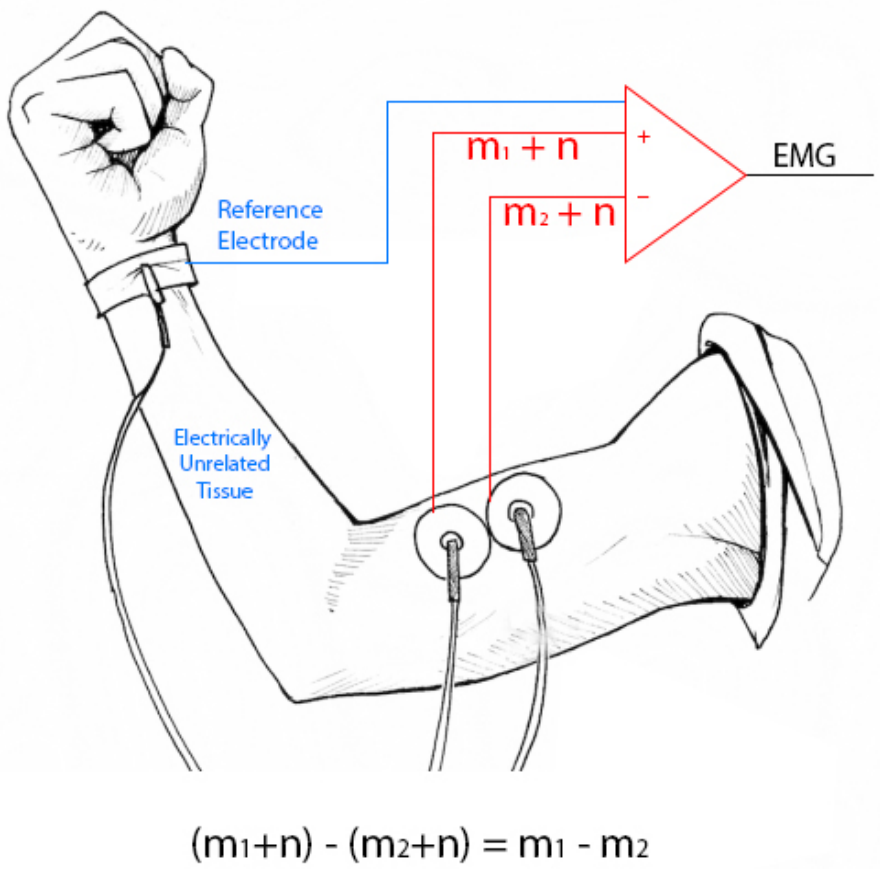

Figure 42. A schematic of the differential amplifier configuration. The EMG signal is represented by " $m$ " and the noise signals by " $n$ ". Adapted from [147].

The bioelectrical signal was amplified with GrassP511 AC ${ }^{\circledR}(20 \mathrm{~V} / \mathrm{mV}$ in trapezius, $5 \mathrm{~V} / \mathrm{mV}$ in deltoids, $10 \mathrm{~V} / \mathrm{mV}$ in biceps, and $20 \mathrm{~V} / \mathrm{mV}$ in flexor carpi radials) and a band pass filter was used with cut off frequencies fixed between $3 \mathrm{~Hz}$ and $1 \mathrm{kHz}$.

The sEMG signal was acquired at $5 \mathrm{kHz}$ sample frequency, by means of $\mathrm{NI}$ USB-6229 card (National Instruments ${ }^{\circledR}$, Texas USA). Specific software was designed and developed for acquiring and analyzing signals in LabView (National Instruments ${ }^{\circledR}$, Texas USA) during this experiment. 


\subsection{Data analysis}

Two parameters were used in order to quantify the signal: RMS and Fmed. RMS values show the level of the physiological activities in the MU during contractions [148] and the frequency values are used to identify muscular fatigue [43], [125].

The data were recorded on a computer in 60 seconds segments, which corresponds to each target position, for post-processing and analysis. Every 60 seconds segment of signal acquired ( $\mathrm{fm}=5 \mathrm{kHz}$ ) can be considered as:

$$
x[n] \quad \text { where } n=0,1,2,3, \ldots \ldots \ldots ., N-1
$$

Being $\mathrm{N}=300000$ datum $(60 \mathrm{sec} \times 5 \mathrm{kHz}$ ). The first parameter calculated was RMS and the equation that defined it is:

$$
R M S=\sqrt{\frac{1}{N} \cdot \sum_{n=0}^{N-1}(x[n])^{2}}
$$

This parameter was normalized with respect to the RMS value obtained in Entrance target with the control instrument for each participant, in order to show results in percentage.

Second parameter, Fmed, was calculated from the PSD, which was computed as an average of the PSD (Welch's periodogram) forming rectangular windows of two seconds. Windows overlapped, thus only 39 windows were applied to compute the PSD:

$$
\operatorname{PSD}[k] \text { where } k=1,2,3, \ldots \ldots \ldots, \frac{L}{2}
$$

Being $L=10000$ datum $(2 \mathrm{sec} \times 5 \mathrm{kHz})$. The median frequency parameter was calculated as: 


$$
\text { Fmed }=D \cdot \frac{f_{m}}{L} \stackrel{\text { where D satisfy }}{\rightarrow} \quad \sum_{k=1}^{D} P S D[k]=\sum_{k=D}^{L / 2} P S D[k]
$$

In order to identify the normality of each group of values, a statistical analysis were carried out using Saphiro-Wilk. T-test analysis was used to identify the significant differences between groups.

\section{Results}

Two questions were discussed during this study in regard to the effect that the PF instrument could cause to surgeon's musculature in laparoscopic procedures: first question is about the muscular activity or effort required by the PF prototype and by the conventional instrument, the second is about the muscular fatigue that both instruments cause. RMS values indicate the muscular activity required in each target of the experiment. Figures 43-46 represent the average values of all the participants, the values are presented as a percentage normalized respect the RMS value in the Entrance target with the control instrument. This target forces the participants to activate all the muscles and it is the first activate position that they performed.

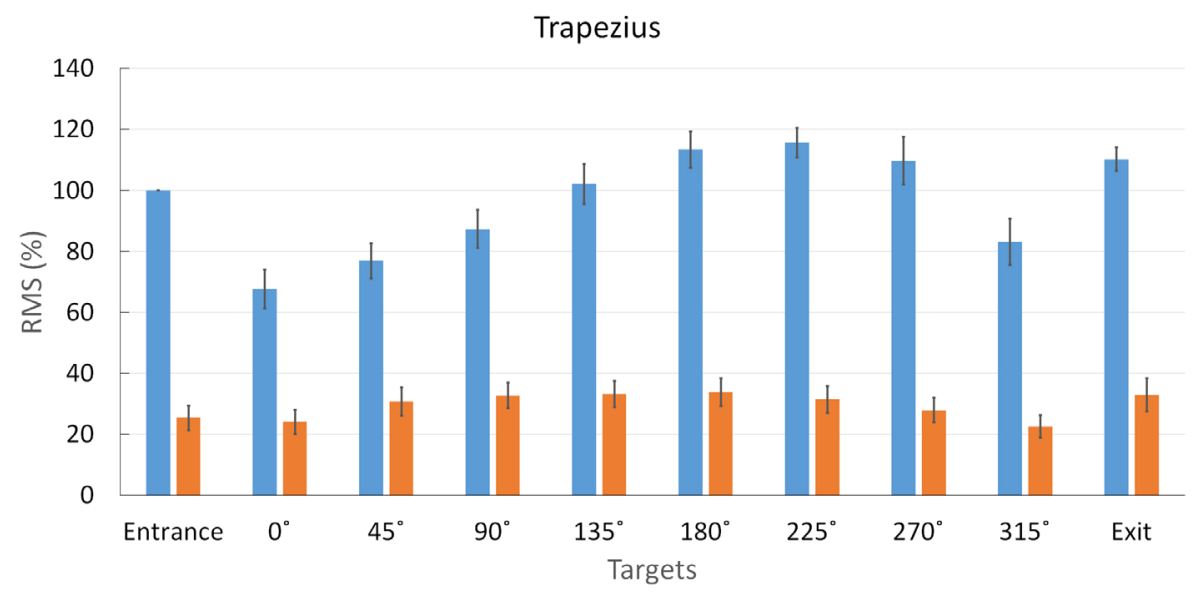

Figure 43. RMS $(\% \pm \mathrm{SE})$ values of trapezius muscle in each target. The values are normalized respect the Entrance target with the control instrument. Control blue bars. Prototype orange bars. 


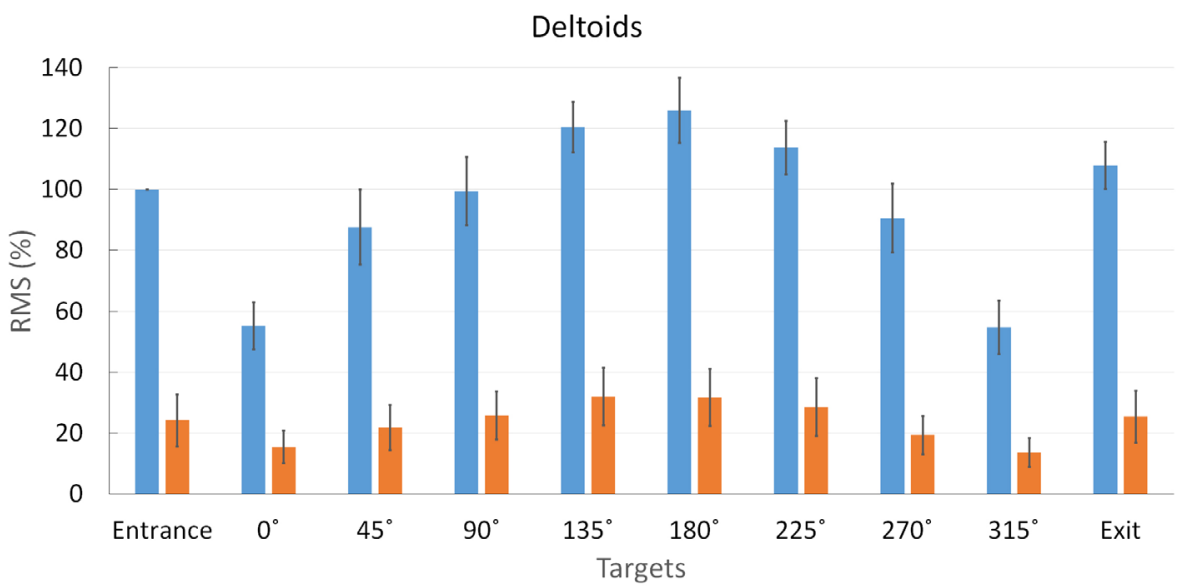

Figure 44. RMS (\% \pm SE) values of deltoids muscle in each target. The values are normalized respect the Entrance target with the control instrument. Control blue bars. Prototype orange bars.

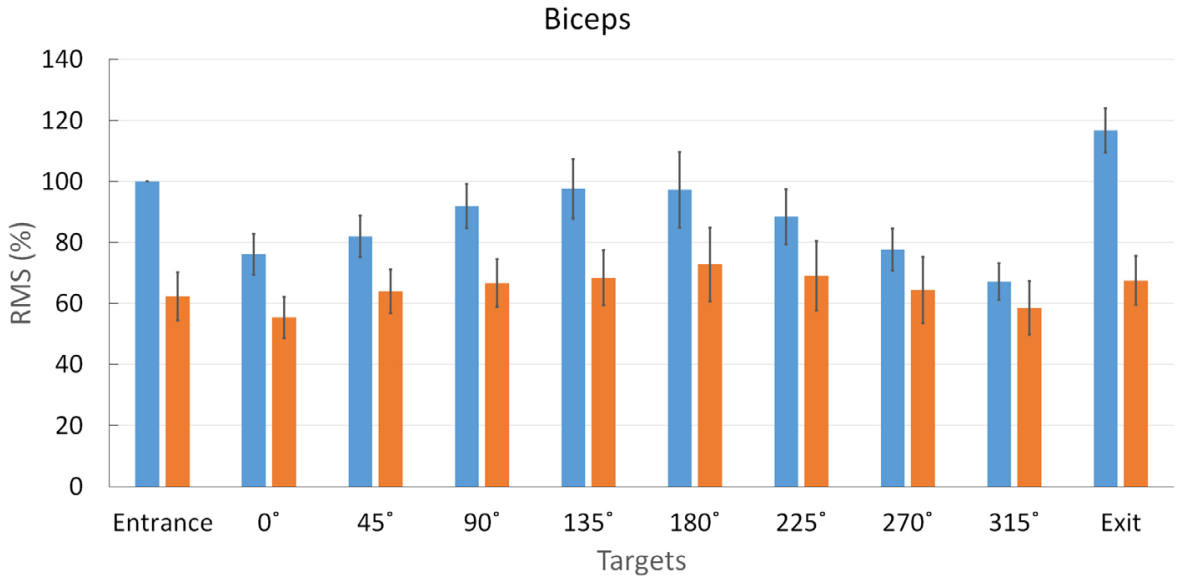

Figure 45. RMS $(\% \pm \mathrm{SE})$ values of biceps muscle in each target. The values are normalized respect the Entrance target with the control instrument. Control blue bars. Prototype orange bars. 


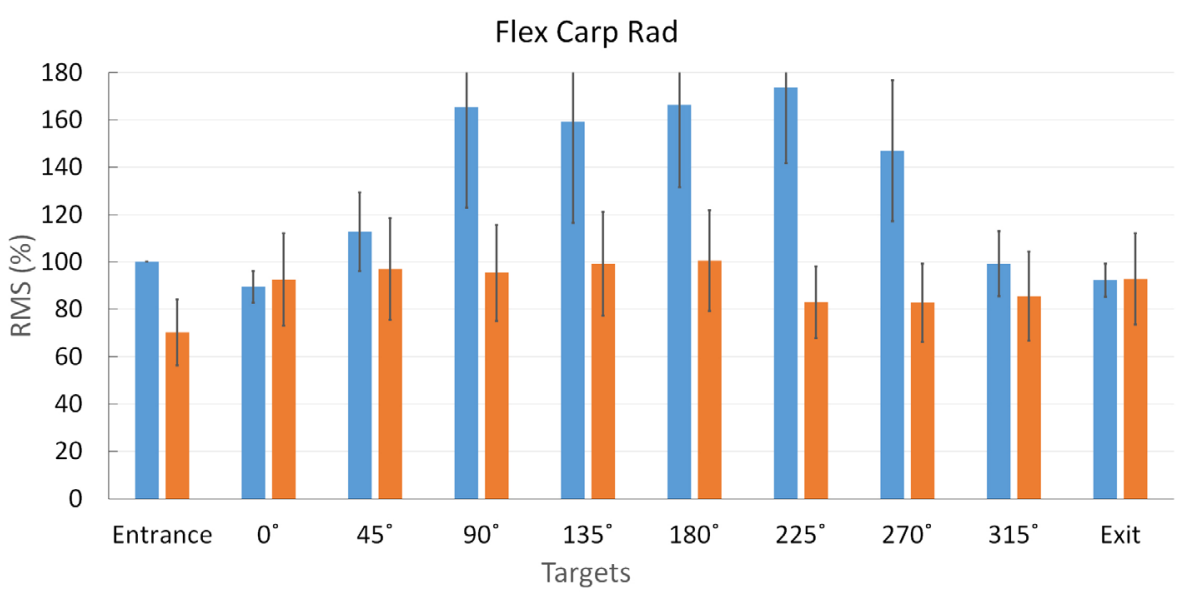

Figure 46. RMS $(\% \pm \mathrm{SE})$ values of flexor carpi radials muscle in each target. The values are normalized respect the Entrance target with the control instrument. Control blue bars. Prototype orange bars. Notice the change of scale.

The most unfavorable positions were generally presented at Entrance and Exit target and in the targets located facing the participants $\left(135^{\circ}, 180^{\circ}, 225^{\circ}\right)$. Despite these positions were also the most unfavorable with the prototype instrument, they presented significant reduction ( $\rho$ value $<0,05$ ) in comparison with the control instrument, with the exception of the Exit target in flexor carpi radials.

The values presented significant differences $(\rho v a l u e<0,05)$ between the PF prototype and the control instrument in almost all the targets evaluated. The targets which difference was not significant were presented in biceps (targets $270^{\circ}$ and $315^{\circ}$ ) and flexor carpi radials (targets $0^{\circ}, 45^{\circ}, 315^{\circ}$ and Exit).

It is important to remark that all these values were normalized to the Entrance target with the control instrument of each muscle. Some targets presented values higher than $100 \%$ because muscular activity at these points was higher than at the Entrance target. The previous evaluations indicated that Entrance target was the appropriate posture to compare with, because it required considerable muscle activation and participants were not tired at the beginning of the experiment.

When the data is evaluated by each participants, the results (Figure 47-50) indicate that the prototype instrument requires a significant more muscular activity by trapezius and deltoids in order to be used but in biceps and flexor carpi radials the prototype does not present any significant benefits. 


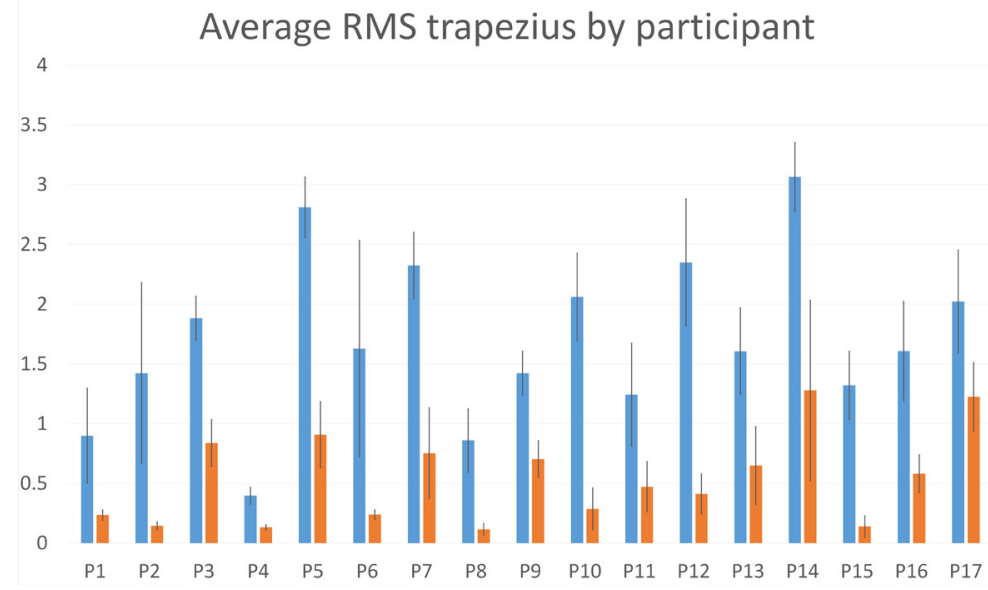

Figure 47. Trapezius RMS values by participant during the whole experiment. Horizontal axis indicate the number of the participant. Vertical axis the value in millivolts $(\mathrm{mV})$.

Control blue bars. Prototype orange bars.

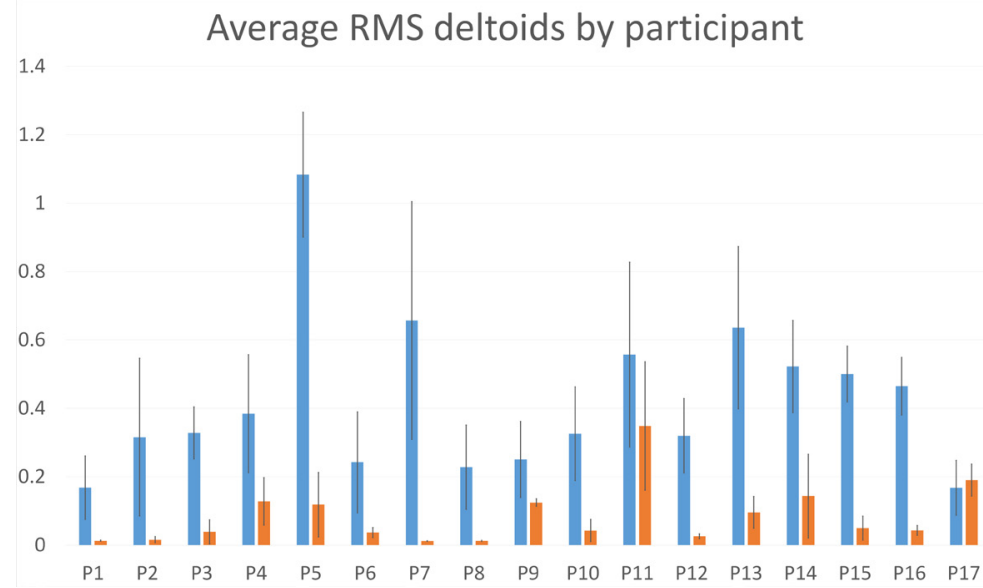

Figure 48. Deltoids RMS values by participant during the whole experiment. Horizontal axis indicate the number of the participant. Vertical axis the value in millivolts $(\mathrm{mV})$. Control blue bars. Prototype orange bars. 


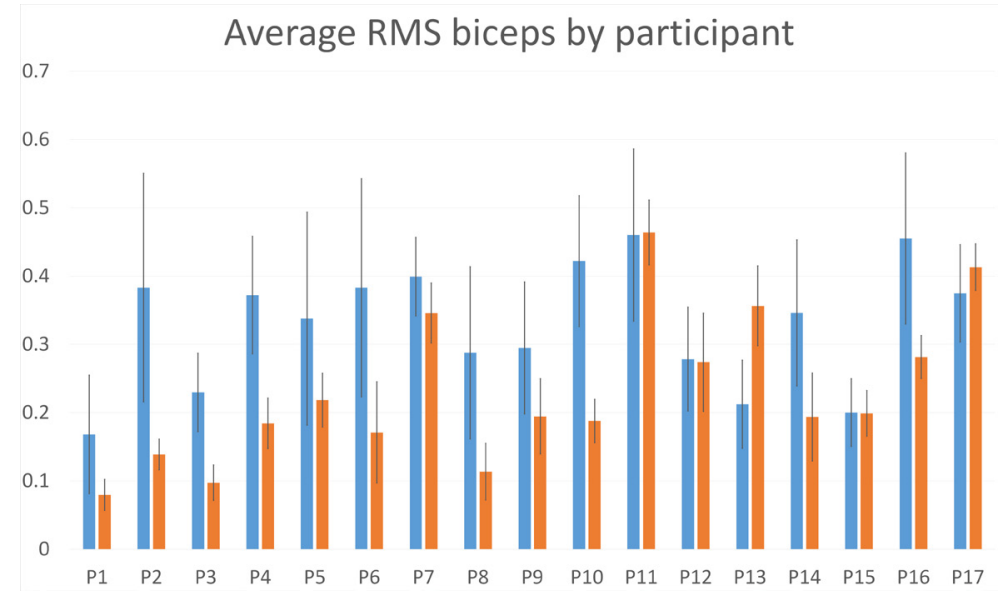

Figure 49. Biceps RMS values by participant during the whole experiment. Horizontal axis indicate the number of the participant. Vertical axis the value in millivolts $(\mathrm{mV})$. Control blue bars. Prototype orange bars.

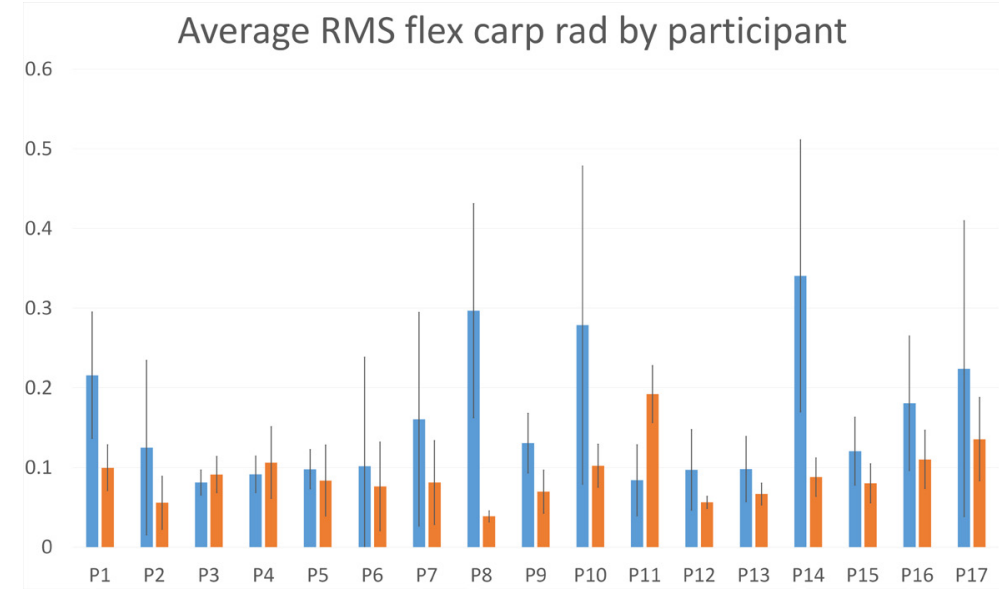

Figure 50. Flexor Carpi Radials RMS values by participant during the whole experiment. Horizontal axis indicate the number of the participant. Vertical axis the value in millivolts $(\mathrm{mV})$. Control blue bars. Prototype orange bars.

In order to identify the muscular fatigue produced during the experiment with the PF prototype and the control instrument. Two similar targets were used as comparison points in the experiment in order to evaluate the muscular fatigue: Entrance and Exit target.

These targets require similar position of the body, but Entrance target is at the beginning of the experiment, with the participant still relaxed, and the Exit target after 10 minutes of effort. 
In order to study the fatigue process, it is required to include a frequency parameter in the evaluation. During this experiment Fmed) and Fm were calculated.

But Fmed has been used because it is less sensitive to added white noise and more sensitive to modifications that occur in the myoelectric signal during sustained voluntary contractions [43], [114], [117], being this parameter more appropriate to assess small signal-noise ratios [118].

The Table 2 presents Fmed values during the Entrance and Exit target of all the participants together. As indicated in the literature, the decrease on Fmed values is an indicator of muscular fatigue [102]. The last column of the table indicates the difference between Entrance and Exit target in each muscle. The values are marked with asterisk $(*)$ if the difference is significant. The results on this table show a significant decrease in all the muscles evaluated with the control instrument. In the case of the PF prototype, this decrease is only significant in deltoids and biceps muscle.

Table 2. Fmed values (Average \pm SD) of PF prototype and control instrument in Entrance and Exit target, and difference between these values. * Significant differences $(\rho<0,05)$.

\begin{tabular}{|c|c|c|c|c|}
\hline & Instrument & $\begin{array}{c}\text { Entrance } \\
\text { target }(\mathrm{Hz})\end{array}$ & $\begin{array}{c}\text { Exit target } \\
(\mathbf{H z})\end{array}$ & $\begin{array}{c}\text { Difference } \\
(\mathbf{H z})\end{array}$ \\
\hline \multirow{2}{*}{ Trapezius } & Control & $60,5 \pm 1,2$ & $57,7 \pm 1,0$ & $2,8^{*}$ \\
\cline { 2 - 5 } & PF prototype & $39,0 \pm 1,3$ & $40,2 \pm 0,9$ & $-1,3$ \\
\hline \multirow{2}{*}{ Deltoids } & Control & $69,0 \pm 1,5$ & $64,4 \pm 1,0$ & $4,6^{*}$ \\
\cline { 2 - 5 } & PF prototype & $59,6 \pm 0,7$ & $58,1 \pm 0,7$ & $1,5^{*}$ \\
\hline \multirow{2}{*}{ Biceps } & Control & $58,7 \pm 1,3$ & $54,1 \pm 1,0$ & $4,6^{*}$ \\
\cline { 2 - 5 } & PF prototype & $50,5 \pm 0,8$ & $45,9 \pm 0,9$ & $4,6^{*}$ \\
\hline \multirow{2}{*}{ Flex Carp Rad } & Control & $77,7 \pm 2,0$ & $65,7 \pm 2,9$ & $11,9 *$ \\
\cline { 2 - 5 } & PF prototype & $54,9 \pm 1,4$ & $53,2 \pm 1,7$ & 1,7 \\
\hline
\end{tabular}

According to JASA method, the decrease of Fmed is not the only important value that has to be considered in order to evaluate fatigue. Figure 51 represent of the Fmed and RMS trend during the experiment. The positions compared were entrance and exit target. The interpretation by JASA of the results are also included. 


\begin{tabular}{ccccc} 
& Instrument & Fmed & RMS & JASA \\
\hline \multirow{2}{*}{ Trapezius } & Control & $\checkmark$ & $\checkmark$ & Muscular fatigue \\
\cline { 2 - 5 } & PF prototype & $\checkmark$ & $\checkmark$ & Force increase \\
\hline \multirow{2}{*}{ Deltoids } & Control & $\checkmark$ & $\checkmark$ & Muscular fatigue \\
\cline { 2 - 5 } & PF prototype & $\checkmark$ & $\checkmark$ & Muscular fatigue \\
\hline \multirow{2}{*}{ Biceps } & Control & $\checkmark$ & $\checkmark$ & Muscular fatigue \\
\cline { 2 - 5 } & PF prototype & $\checkmark$ & $\checkmark$ & Muscular fatigue \\
\hline \multirow{2}{*}{ Flex Carp Rad } & Control & $\checkmark$ & $\checkmark$ & Force decrease \\
\cline { 2 - 5 } & PF prototype & $\checkmark$ & Muscular fatigue
\end{tabular}

Figure 51. Result of Fmed and RMS and the interpretation by JASA of the results presented by all the participants together. Upward arrow indicates an increase between entrance and exit targets. Downward arrow indicates a decrease between entrance and exit targets.

Despite there are several participants which present muscular fatigue according to JASA parameters (Table 3), RMS and Fmed parameters presented significant differences $(\rho<0,05)$ between entrance and exit target in just a fraction of these participants.

Table 3. JASA evaluation by participant. Results indicate muscular fatigue (MF), force increase (FI), force decrease (FD), and muscle recovery (MR). $\left(^{*}\right)=\rho<0,05$

\begin{tabular}{|c|c|c|c|c|c|c|c|c|}
\hline & \multicolumn{4}{|c|}{ Control } & \multicolumn{4}{|c|}{ PF Prototype } \\
\hline & trapezius & deltoids & biceps & flexor & trapezius & deltoids & biceps & flexor \\
\hline P1 & MF & FD & MR & MF & $\mathrm{FI}$ & $\mathrm{FI}$ & $\mathrm{FI}$ & MF \\
\hline P2 & FD & FD & FD & MF & MF & MF & $\mathrm{FI}$ & FD \\
\hline P3 & MF* & FD & FD & $\mathrm{MF}^{*}$ & MF & $\mathrm{FI}$ & $\mathrm{FI}$ & MF \\
\hline P4 & $\mathrm{FI}$ & $\mathrm{FI}$ & $\mathrm{FI}$ & FD & $\mathrm{FI}$ & $\mathrm{FI}$ & $\mathrm{MF}^{*}$ & $\mathrm{Fl}$ \\
\hline P5 & $\mathrm{FI}$ & MF* & MF* & FD & MF & FD & $\mathrm{FI}$ & MF* \\
\hline P6 & $M F^{*}$ & FD & $\mathrm{MF}^{*}$ & $M R$ & FD & FD & FD & MF \\
\hline P7 & MF & MF & FD & FD & $\mathrm{FI}$ & MF & $\mathrm{MF}^{*}$ & MF \\
\hline P8 & $\mathrm{FI}$ & $\mathrm{MF}^{*}$ & $\mathrm{MF}^{*}$ & $\mathrm{FI}$ & $\mathrm{FI}$ & $\mathrm{MF}$ & FD & $\mathrm{FI}$ \\
\hline P9 & MF* & FD & MF & MR & MF & FD & FD & $\mathrm{FI}$ \\
\hline P10 & MF* & $\mathrm{FI}$ & $\mathrm{FI}$ & FD & $\mathrm{Fi}$ & $\mathrm{FI}$ & FD & FD \\
\hline P11 & MF & FD & FD & FD & $\mathrm{FI}$ & MR & $\mathrm{MF}^{*}$ & MF \\
\hline P12 & MF & MF & $\mathrm{MF}^{*}$ & $\mathrm{MF}^{*}$ & $\mathrm{FI}$ & $\mathrm{MF}$ & $\mathrm{MF}^{*}$ & $\mathrm{MF}$ \\
\hline P13 & FD & FD & MF & FD & MR & MF & FD & $\mathrm{FI}$ \\
\hline P14 & FD & $\mathrm{FI}$ & $\mathrm{MF}^{*}$ & $\mathrm{FI}$ & $\mathrm{FI}$ & $\mathrm{FI}$ & $\mathrm{MF}^{*}$ & $\mathrm{MF}$ \\
\hline P15 & FD & FD & MF & MR & FD & FD & FD & $\mathrm{MF}$ \\
\hline P16 & MF & $\mathrm{FI}$ & $\mathrm{MF}^{*}$ & FD & FD & MF & $\mathrm{MF}^{*}$ & $\mathrm{FI}$ \\
\hline P17 & $\mathrm{FI}$ & $\mathrm{MF}^{*}$ & FD & $\mathrm{MF}$ & $\mathrm{FI}$ & $\mathrm{MF}$ & $\mathrm{MF}^{*}$ & $\mathrm{FI}$ \\
\hline
\end{tabular}




\section{Discussion}

The fact that the conventional laparoscopic instruments force surgeons to move to non-neutral postures has been widely study [61], [65], [69], [149], [150]. The scarcity of neutral postures complicates the procedure for the surgeons and can produce disorders in their upper limbs and backs. The present study was designed to identify the effect produced by the PF characteristic in the muscles trapezius, deltoids, biceps and flexor carpi radials. To reach this goal three issues have been addressed.

This study tries to reach a deep understanding of the effects that a PF instrument could have on surgeons' upper body. For this purpose, in this experiment the participants performed a comparative muscular evaluation using the PF prototype and conventional instrument as a control. The control instrument was a conventional pistol-grip handle with a fixed handle-to-shaft angle.

The results obtained with the control instrument show the highest activity values in trapezius (Figure 43 and Figure 47) and deltoids muscles (Figure 44 and Figure 48) and the most unfavorable targets for these muscles were the Entrance and Exit target and the $135^{\circ}, 180^{\circ}, 225^{\circ}$ and $270^{\circ}$. The cause of the high activity values during these targets is mainly the design of the instrument, because in these targets the fixed handle-to-shaft angle forces the participant to abduct and stretch the arm to reach the targets [37], [123], [151].

With the PF prototype, the results in this critical positions present the highest differences. The PF prototype reduced the muscular activity required on trapezius critical positions between $70 \%-74 \%$ in comparison with the control instrument (Figure 43) and these differences are presented in deltoids too, reducing the muscular activity in most critical positions between $73 \%$ - 76\% (Figure 44).

The PF prototype also presented differences in biceps (Figure 45 and Figure 49) and flexor carpi radial muscle (Figure 46 and Figure 50), but these differences were not as bigger as presented by trapezius and deltoids. In biceps muscle the critical targets for the control instrument were presented in $90^{\circ}, 135^{\circ}, 180^{\circ}$, Entrance and Exit. 
The results obtained by the PF prototype in biceps muscle were between $25 \%$ $42 \%$ lower than the control instrument (Figure 45). In flexor carpi radials, the critical targets for the control instrument were presented in $90^{\circ}, 135^{\circ}, 180^{\circ}, 225^{\circ}$ and $270^{\circ}$, the reductions in these targets presented by the PF prototype range between $37 \%$ $52 \%$ (Figure 46).

The low reduction presented by biceps and flexor carpi radials can be explained by two different reasons. In the case of the biceps muscle, it is in charge of the forearm flexion and this positions is required to introduce and maintain the instrument inside the simulated abdomen, and this is a position forced by the point of entrance, not for the instrument. In flexor carpi radials the reason is different, this muscle is the responsible of abduct and flex the wrist, and the ring handle presented in both instrument forces the participants to perform these movements to introduce the fingers in the rings.

Overall, these results indicate that, if a surgeons needs to use the conventional pistol grip handle with a fixed handle-to-shaft angle, in terms of muscular activity or effort, is preferred to reach targets located between $0^{\circ}$ and $45^{\circ}$ of the trocar entry. But if they use an instrument that allows PF, they can reach every single target inside the abdominal wall without great displacements of their upper limbs, reducing the muscular activity even in the critical targets because the PF prototype allows to relax the musculature once the target is reached in contrast to the conventional fixed instruments that force the surgeons to maintain abducted and flexed positions.

In 2004, a needle driver with variable handle-to-shaft angle was evaluated [37]. The needle driver allows fixing their handle-to-shaft angle in $0^{\circ}, 40^{\circ}$ or $80^{\circ}$ to perform the suture, the goal of the study was to identify the optimal ergonomic handle-to-shaft angle for suturing. The instrument evaluated by the authors has a different configuration but they also identified the fixed handle as the cause of great abduction at the shoulder and acute flexion of the elbow. The fact that they did not find significant differences between the angles $40^{\circ}$ and $80^{\circ}$ can be caused because each surgeon have their own optimal handle-to-shaft angle, and the use of the PF could be an effective solution to increase the comfort of the surgeons. In 2007, an study evaluating a rotatable instrument that can rotate $360^{\circ}$ transversely to the shaft of the handle [151]. According the authors, the rotatable instrument allows some reductions of muscular activity in biceps and flexor carpi radials but they did not found these reductions on trapezius and deltoids. 
The results presented by the PF prototype reflect differences with the rotatable instrument presented by $B$. Steinhilber. The rotatable instrument does not reduce the muscular activity in trapezius and deltoids muscles which are considered decisive to the reduction of the musculoskeletal disorders in back and shoulder, however the PF instrument presented a considerable improvement for these muscles. The effectiveness and precision of the rotatable instrument depends on the target location, in the case of the PF instrument the reduction of the muscular activity is presented in any position but the precision of the instrument was not addressed in this study. The PF prototype limitations made impossible to evaluate its precision during the same experiment, but this is an important issue for future researches because the test observation suggest that the precision with the PF instrument need to be improved.

The results obtained suggest that the concept of PF applied to new laparoscopic instruments could reduce the muscular activity in the muscles evaluated. Interestingly, the targets considered critical to reach with the control instrument are which presented highest differences. The low RMS values in the critical targets, added to the low spread of results presented by the PF prototype in the whole set of targets, suggest that the activation of the muscle using the PF prototype does not depend on the target location and the handle-to-shaft variable angle of the PF prototype is the cause of the reduction on these values.

With respect to the muscular fatigue, apparently both instrument caused muscular fatigue to the participants but with some differences. JASA was used in order to identify muscular fatigue. JASA method was presented in 1996 by A. Luttmann [121] with the aim to popularize a method that discriminates between fatigue-induced and force-related changes in the EMG signal.

According to this method, the muscular fatigue is produced if the EA generated with the muscle is increasing and Fmed is decreasing. Considering that the RMS obtained in this study is similar as the EA presented by Luttmann and previously used as indicator of fatigue [22], [77], [101], [116] then, JASA method can be used to identify muscular fatigue during this experiment. 
In the study presented, Table 2 shows the Fmed values of both instruments on Entrance and Exit targets. Difference between entrance and exit are indicated in the fourth column, if this value is positive means that the Fmed is decreasing in that muscle, which is the first indicator of fatigue. According to the general results, all the muscles are fatigued by the experiment except the trapezius muscle when the experiment is performed by the PF prototype.

Because JASA requires an activity value too in order to identify muscular fatigue, in Figure 51 the Fmed and RMS values differences between entrance and exit positions are presented. This figure corroborates greatest part of the results presented in Table 2 . The trapezius muscle with the PF prototype is not the only one that does not present muscular fatigue, according to JASA, the control instrument does not present muscular fatigue in flexor carpi radials.

In order to widely understand the muscular fatigue presented during this experiment, the JASA results for each participant are presented in Table 3. According to the results, there were no participants that presented a significant muscular fatigue in trapezius and deltoids while using the PF prototype. In biceps muscle and flexor carpi radials the amount of participants presenting significant muscular fatigue were similar with both instruments.

Broadly speaking, the results suggest that muscle fatigue is produced with both instruments, but the PF prototype considerable reduce it in two important muscles: trapezius and deltoids. Despite biceps and flexor carpi radials fatigue was not reduced according to JASA, is important to notice that this method does not consider the amount of muscular activity as an indicator to take in consideration. Nevertheless, according to the literature, muscular fatigue is directly related to the force exerted [31], for this reason it could be concluded that the higher values of muscular activity presented by the control instrument will make it produce fatigue faster than the PF prototype. 



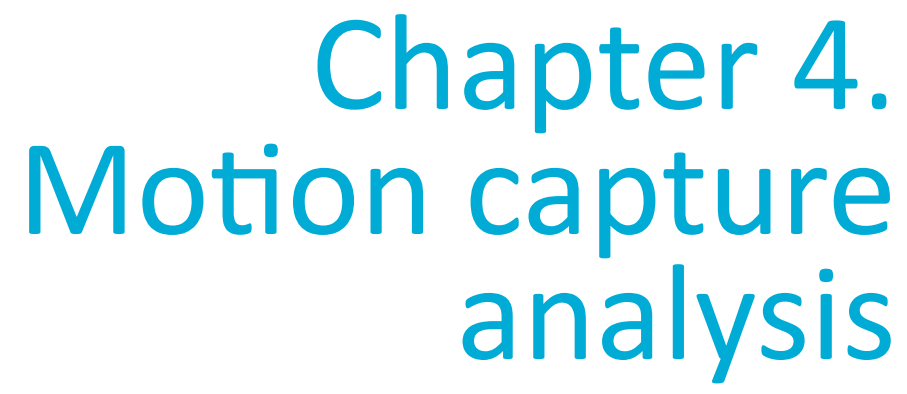




\section{Introduction. Motion capture in ergonomic studies}

\subsection{Critical movements in laparoscopic surgery}

Due to the fixed entry point used in laparoscopic surgery and the limitations of this produce, the laparoscopic surgery presents a number of ergonomic difficulties for surgeons [76]. During these procedures, surgeons are required to adopt awkward positions of the arms, hands, and fingers that can result in pressure point injury, nerve compression, vertebral disk injury, pain and muscle fatigue [19], [36], [76], [82]. Some of the risk factors for developing musculoskeletal disorders in laparoscopy are: static muscle loading, repetitive fine motor handling techniques, extreme joint angulations as well as work duration and load [21].

The ideal position for the laparoscopic surgeon was presented by U. Matern and P. Waller in 1998 [64]. The arm should be slightly abducted, tilted abnormally backward, and rotated inward at shoulder level, and the elbow should be bended at about $90-120^{\circ}$ (Figure 52).

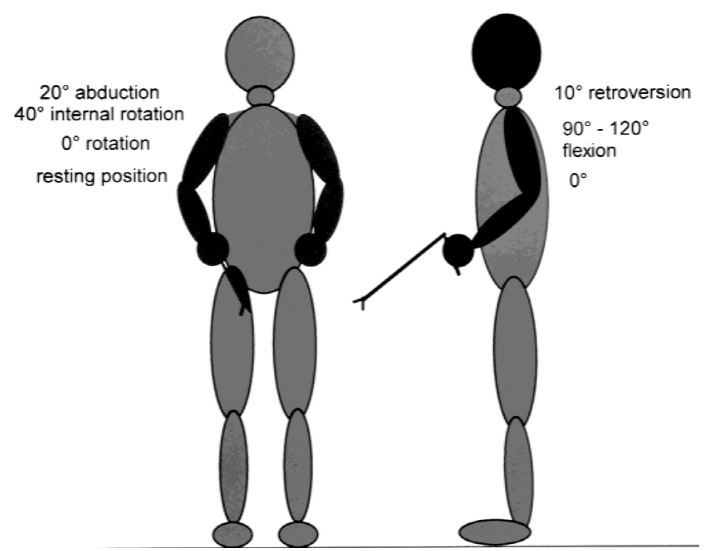

Figure 52. Ergonomically ideal position for the laparoscopic surgeon. Adapted from [64]. 
Unfortunately, this ideal position is not commonly adopted during the procedure and usually their posture is more similar to the presented in Figure 53.

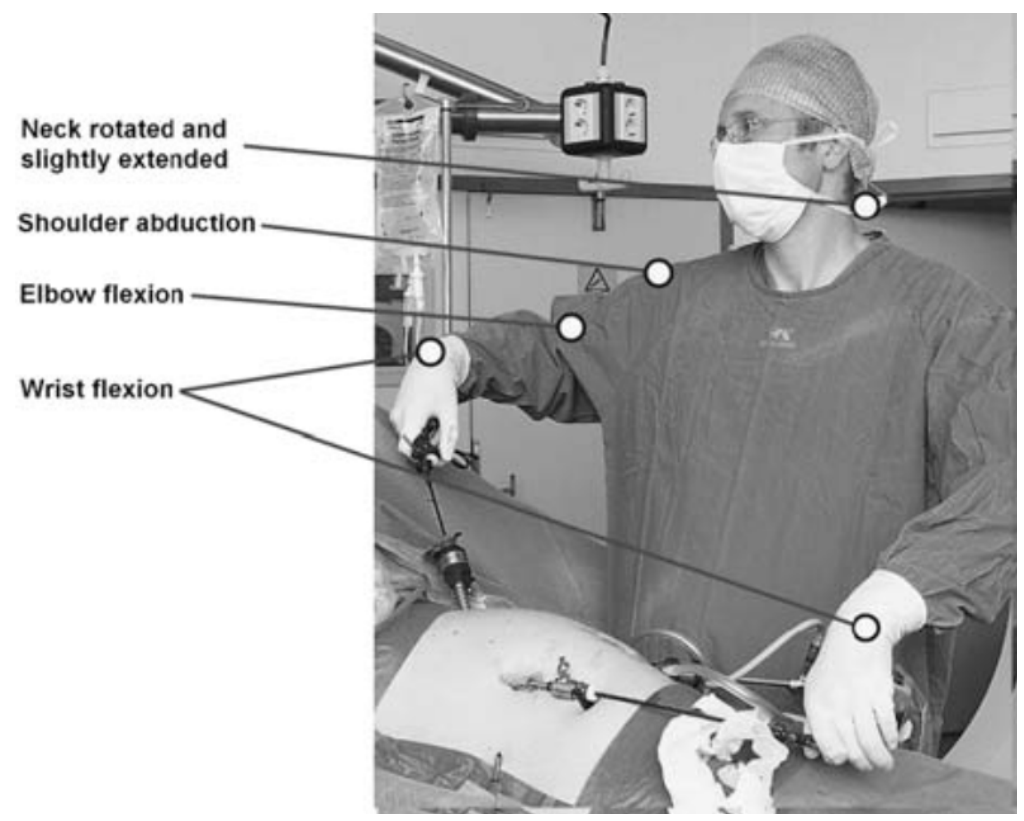

Figure 53. Static position during surgery. Adapted from [39].

Pain and injury due to occupational workload is common in surgeons, compared with open surgical techniques, laparoscopic surgery involve a more static posture of the neck and trunk, and more frequent awkward repetitive motions of the upper extremities. Shoulder motion is affected by the nature of laparoscopic surgery, the fixed position of the trocar forces the surgeons to move their upper extremities into awkward positions to manipulate tissues at different angles inside the body cavity [21]. The instruments pivot about the trocar and the surgeon is forced to abduct the arm and flex, pronate and supinate the wrist in order to actuate the handle [62]. These difficult maneuvers, combined with the mechanical inefficiency inherent in the design of the instruments, can lead to significant upper extremity muscular effort and strain even among experienced laparoscopic surgeons [62].

An interesting study by L. Aitchinson et al. published in 2016 [76] showed the critical movements for laparoscopic surgeons. During 5 months the team recorded 128 different procedures developed by laparoscopy.

The study showed interesting results as, for example, that the visualizing of the monitor is the primary impact on the neck for the surgeon because they spent a median of $98 \%$ of the time with the neck in a single position observing the monitor. 
For the shoulder, the median most extreme shoulder angles during trocar insertion were $82^{\circ}$ of flexion, $50^{\circ}$ of extension and $61^{\circ}$ of abduction. For the elbow, the nondominant arm is consistently in more extreme flexed positions compared to the dominant arm $\left(>120^{\circ}\right)$.

As explained in the introduction, there is a category in the ergonomic research method called observational methods. This category used a technique called MoCap to record the movements of a character by means of sensors and cameras that measure certain variables [47], [49]. MoCap, by definition, is the process of recording a live motion event and translating it into usable mathematical terms by tracking a number of key points in space over time and combining them to obtain a single three-dimensional representation of the human body motion [152]. In brief, it is the technology that enables the process of translating a live performance into a digital performance [153]. There are two main families that classify the different MoCap technologies according to the kind of sensors they use: non vision-based sensor technology (NVBST) and vision-based sensor technology (VBST).

The NVBST uses sensors attached to the subject body that provide diverse information. Some examples of NVBST are the inertial sensors (measures acceleration, angle, vibration, movement, and multiple DoF), magnetic sensors (measures speed, rotational speed, linear position and linear angle and position), electrical sensors (measures the EA of muscle contraction during gait).

The use of the VBST has becoming popular in the last decades. This technology uses optoelectronic sensors and cameras to track the human movement and thus estimate the movement parameters and position. The optoelectronics sensors may or may not require the use markers to track movements. Markers are attached to the body to represent the skeletal segments and joints, the optical system tracks the markers and obtains the body segments and the position and orientation of each joint. In the markerless systems, the image features such as color, edges, shapes, and/or depth are used to interpret the motions [154].

In this work was used a VBST, because of the importance of no interfere in the surgeon's movements. Because in the operation room they cannot wear sensors, and was important to keep the conditions in the simulation area as realist as possible.

Although most of the basic principles of optoelectronic sensors have been known 


\subsubsection{The VBST system}

for decades, the industrial applications are relatively new. An optoelectronic sensor is a device that produces an electrical signal proportional to the amount of light incident on its active area. Three are the main techniques that use these sensors [154]: Marker-based, Depth-based and Contrast-based.

\subsubsection{Marker-based}

The marker-based VBST technique tracks human movement through the use of cameras that capture the identifier points of the human body. These markers are attached to different parts of the body with the aim to obtain an accurate representation of each skeletal section.

\section{The VICON system}

The VICON (Variable Independent Control) System, created by Vicon Motion Systems Ltd (Oxford, UK) uses a series of cameras to track the movement of the markers located in different parts of the body. This system requires, in its simple configuration, at least 8 cameras (Figure 54) with multiple high-speed processors that perform real time image processing, and a high number of markers (depends on the number of sections of the body to track).

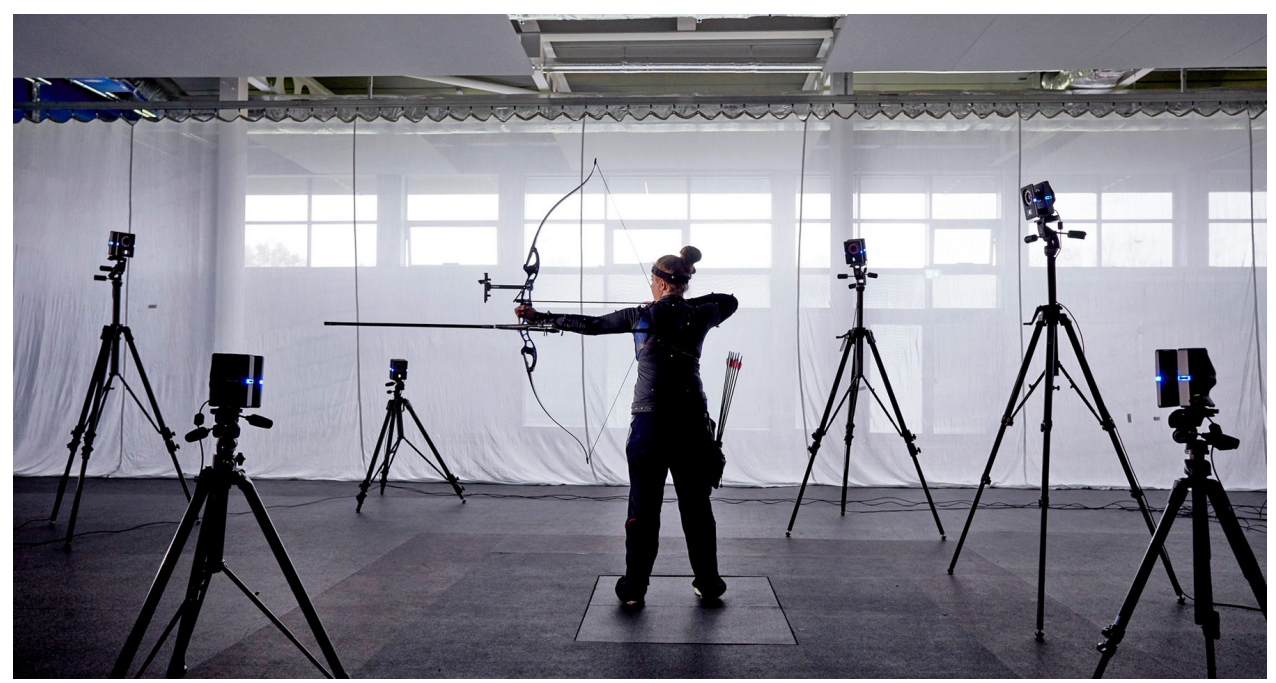

Figure 54. Basic VICON setup to evaluate an archer motions. Acquired from Engadget magazine. 
The high accuracy and precision registered by the VICON system converts it into the gold standard of the VBST systems. Its characteristics has been widely compared with other MoCap systems in order to found a technology that surpass the qualities of the VICON system. Nevertheless the VICON system presents the lowest maximum error of the systems on the market [155]: maximum error 5,57 mm (avoiding distances that the system cannot evaluate: 0 and $10 \mathrm{~mm}$ ); maximum degrees error 4,632 .

This system has been used to track movements in different areas since gait analysis for rehabilitation [156] or movements record for professional athletes in different sports [157]-[159] until surgeons and new instruments for laparoscopic surgery in simulation environments [160]-[162]. Probably the major drawbacks of this system, without considering the price, is the amount of cameras that it requires and which make it too difficult to transport it in order to track different environments.

\section{Other marker-based methods}

VICON system is the most popular marker-based system, but is not the only one. Other systems also use markers to evaluate the movements and limb displacements of subjects.

Two main families according to the type of markers are included on the markerbased system [77]: The first one uses passive markers to record the movements, it markers require cameras that incorporate strobe light sources (LED rings around the camera lens). This type of cameras captures the light returned from the highly reflective markers and process the data. This is the case of VICON system; On the other hand, the active markers don't need this type of advanced cameras and the camera systems is limited to record the light emitted by the markers. The light is generated by small LEDs inside the markers. Both, active and passive markers, are attached to the subject body to track the movements.

These families of markers present advantages and disadvantages: The anatomical location of each marker used in an active marker system is immediately known by the cameras because the markers are sequentially pulsed by a controlling computer while in the passive markers, the user should indicate to the system some parameters to improve the system acquisition, although automatic tracking algorithms have been developed to improve this process. Probably one of the main drawback is presented on the active markers because it requires wires to power and control the LED light inside the markers and it may increase the possibility for subjects distraction and motions alteration [77]. 


\subsubsection{Depth-based}

Depth cameras measure the range information between the camera and the object. Being this system more convenient for three-dimensional model construction, object tracking, movement detection, etc. [163].

\section{The KINECT system}

In November of 2010 the Kinect V1 sensor (Microsoft, USA) was released. The launch of this sensor enriched the depth-camera device options with it low consumer price, compact size, and the capability to capture depth and image data at video rate [163]. This device consists on a RGB camera, a depth sensor and a multi-array microphone. The device projects patterns consisting of many stripes at once, allowing the acquisition of multiple samples at once. It has several advantages: it can capture color and depth images independent of lighting conditions, resolve silhouette ambiguities in pose, and is color and texture invariant [154], [163], [164].

Since the apparition of the Kinect V1 sensor, a wide range of experiments have been reported with the aim to obtain a marker-less system that provides similar accuracy and precision to the VICON MX system with the qualities of the KINECT system as very low price, ease to carry and non-requirement of markers. One of the most cited articles on this purpose was carried out by T Dutta [165] in 2012. Dutta evaluated the Kinect V1 sensor for measurement in the workplace comparing the results with VICON System. During this experiment, the authors create four cubes with a front green face, over these cubes the author located spherical reflective markers. Each marker was moved to different position on the set, collecting a total of 104 different target positions with both systems. The results are presented in the Table 4. With these results the author considers that, with further development, the Kinect system may be used as a portable 3D MoCap system for performing ergonomic assessments. Similar conclusions were reported by other authors, referring the accuracy and sensitivity of the systems as the main characteristic to improve if they want to have in Kinect V1 a powerful alternative to conventional MoCap system [154], [166]-[170].

Table 4. RMS error average (SD). First column compare the Kinect results with the real location of the cube. Second column compare the results with the VICON system results.

\begin{tabular}{|c|c|c|}
\hline & Versus reality & Versus VICON \\
\hline X axis & $16,9 \mathrm{~mm}( \pm 29,9)$ & $6,5 \mathrm{~mm}( \pm 4,8)$ \\
\hline Y axis & $34,8 \mathrm{~mm}( \pm 76,5)$ & $10,9 \mathrm{~mm}( \pm 5,9)$ \\
\hline Z axis & $14,1 \mathrm{~mm}( \pm 25,0)$ & $5,7 \mathrm{~mm}( \pm 4,2)$ \\
\hline
\end{tabular}


Trying to improve the accuracy results of the Kinect v1 sensor, some authors tracked the movements and body shapes with two or more Kinect $V 1$ sensors recording at the same time. In 2011, A. Maimone and H. Fuchs [171] presented a conference report using a multiple Kinect V1 system to create a telepresence system. One of the most interesting results reported in this experiment is about the interference problem because the patterns projected by each sensor caused interference on the other one (Figure 55). This interference occurs because each sensor projects the same pattern of points on the same wavelength. It allows the sensor to recognize the pattern of the other sensors in the same room, creating problems to recognize its own pattern.

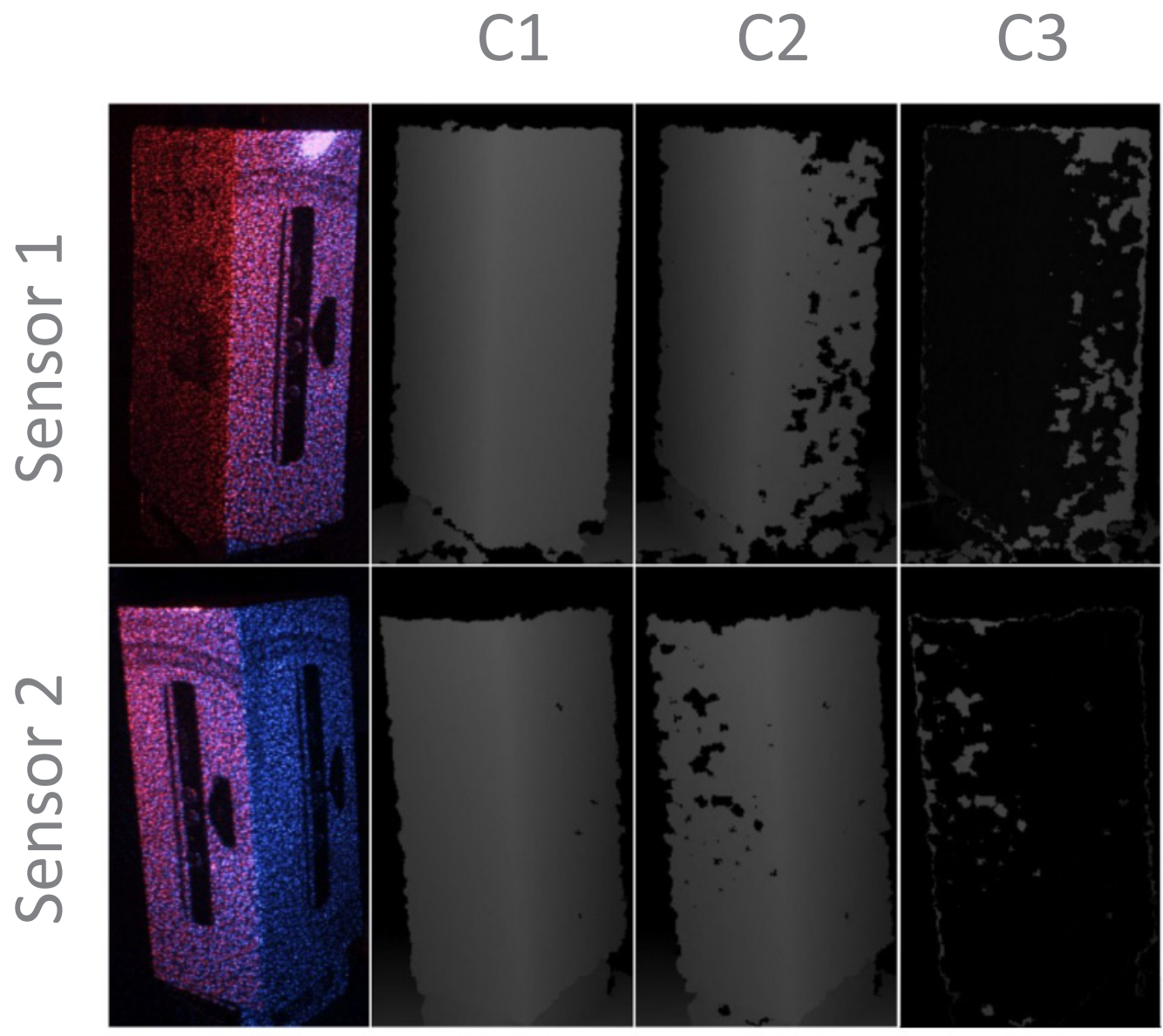

Figure 55. Kinect interference problem. The column C1 shows the depth images of each

sensor with no interference. In the column C2 the depth images of each camera in a system composed by two cameras reflect the interference problem. The column C3 reflect the missed information caused by the interference. Adapted from [171].

In 2014 a new upgrade of the Kinect sensor became available (Kinect V2). This upgraded present improvements in the camera, the depth sensor and the number and place of the nodes. 
In 2015, L. Yang et al. [163] published a wide ranging evaluation of the depth sensing capability of the Kinect $\mathrm{v} 2$ sensor. They evaluate the individual characteristics of Kinect V2 sensor and also a system composed of multiple sensors. The conclusions presented indicate that the Kinect V2 sensor has an acceptable performance to be applied in medical fields. One of the problems reported with this technology is that when two Kinect v2 sensors are positioned towards each other, the area around the camera would disappear in the depth images captured by both two sensors. This phenomenon occurs because the camera interference with the IR light from the other sensor as in the case the Kinect V1.

\section{Other depth-based methods}

Systems with depth-based sensors use depth segmentation and computer vision algorithms to detect and track human body from the sequences of depth images. This tracking method generally requires special cameras or devices that tracks the environment and recognizes variations in depth parameters [154]. Kinect sensor use this kind of tracking method, but is not the only one, some other systems with tracking methods based also in depth contrast are: Orbbec Astra (Orbbec3D, Seattle, USA) Intel RealSense (Intel, Santa Clara, USA), ZED Stereo Camera (Stereolabs, San Francisco, USA), DUO MC (Code Laboratories, Nevada, USA), Leap Motion Controller (LeapMotion, San Francisco, USA).

\subsubsection{Contrast-based}

Contrast-base sensor systems may track a specific color on the video recorded, this color can be on the participant's clothes or in a marker attached to their body. This tracking method requires the use of cameras, preferable with high color-contrast to recognize each variation in the image colors, and software to identify each pixel of the image and follow the time-variations.

\section{The TRACKER system}

Tracker Video Analysis (Cabrillo College, California, USA) is a Java-based video analysis tool developed by the Open Source Physics Project. This tool allows to track and analyze an image or video clip to determine multiple variables.

The program is designed to be used in physics experiments in order to easily estimate for example the acceleration and velocity of an object. A typical video modelling experiment requires a digital video file, a calibration of the scale and a definition of coordinate axes just as for traditional video analysis [172]. This software has been used before to track the trajectories of instruments during laparoscopic surgeries with positives results [173] and it is an affordable solution. 
The Tracker software allows to track positions manually or automatically, recording data of position, angulation, velocity and acceleration among others. The software indicate in real time, the data you are interested on, in the example of Figure 56 , the system is tracking the position of the dropped ball and the graphs represent the position in $X$ (above) and $Y$ (below) over the time.

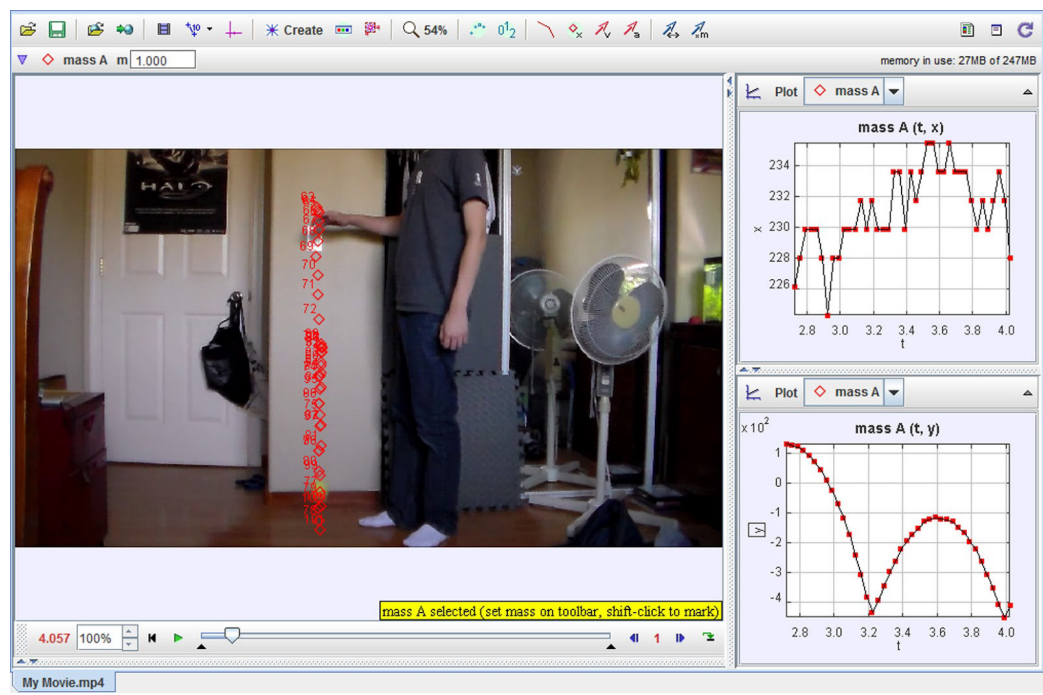

Figure 56. Tracker Video Analysis Software. In this image, the software is tracking the position and time of the ball. Red rhombus in the picture mark the position of the ball on each frame.

\section{Other contrast-based methods}

Some examples of this kind of software are Easy Java Simulations (Universidad de Murcia, Murcia, Spain), WINanalyze (Mikromak, Berlin, Germany) T-WREX (Univ. of California, California, USA) or Kinovea (Kinovea.org, France). As explained above, this kind of systems track colours on markers or skin. Some of this software are open source and is not necessary to use special cameras. It is necessary to carefully follow the record once is finished because sometimes the system losses the markers.

One of these software, the Iconico Screen Protractor, has been previously used to evaluate the surgeon's movements during laparoscopic surgeries [21], [76]. During this study, the authors evaluated the angles of neck, shoulder and elbow during different procedures. The Iconico Screen Protractor was consider as a useful instrument to evaluate the movements inside the operation room because its accuracy and its low interference with the surgeon's movements. 


\subsection{Introduction to the experiment}

Despite all the benefits presented by the MAS techniques, during these procedures surgeons are forced to adopt awkward postures and exert substantially higher muscle force on fingers, hands, wrist and arms during MAS procedures [69], [174].

Awkward postures also affect surgeons after operations. In fact, one of the leading causes of surgeon post-operation pain or numbness is the non-neutral postures adopted during laparoscopy [70]. A poor ergonomic posture accelerates the muscle fatigue and pain process because, outside the neutral range, muscles require more energy to generate the same contractile force than in neutral positions [142].

Previous authors tried to solve the complications of these procedures increasing the DoF in the end effector [69], [72], [175] obtaining positive results but not completely solving the scarcity of neutral postures in wrist, arm and forearm.

The goal of this experiment was to identify the effect that the PF prototype have in the surgeon's posture during a laparoscopic simulation and the differences between expert surgeons and novices. Angles of abduction, adduction, flexion and extension were measured in order to identify the average angle and maximum angles performed during the simulation.

The abduction and adduction motions occur within the coronal plane and involve medial-lateral motions of the limbs. Abduction moves the limb laterally away from the midline of the body, while adduction is the opposing movement that brings the limb toward the body or across the midline [176].

Arm abduction is raising the arm at the shoulder joint, moving it laterally away from the body, while arm adduction brings the arm down to the side of the body (Figure 57). 


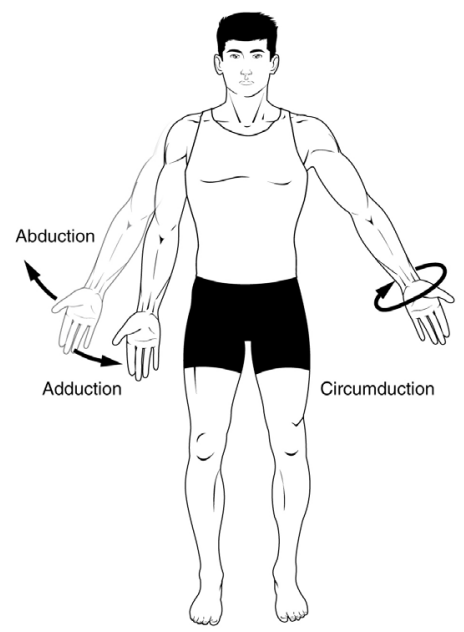

Figure 57. Angular movements: abduction, adduction, and circumduction of the upper limb at the shoulder. Adapted from [176].

Flexion and extension are movements that take place within the sagittal plane and involve anterior or posterior movements of the body or limbs. In the limbs, flexion decreases the angle between the bones (bending of the joint), while extension increases the angle and straightens the joint [176]. For the arm, all anterior-going motions are flexion and all posterior-going motions are extension (Figure 58).
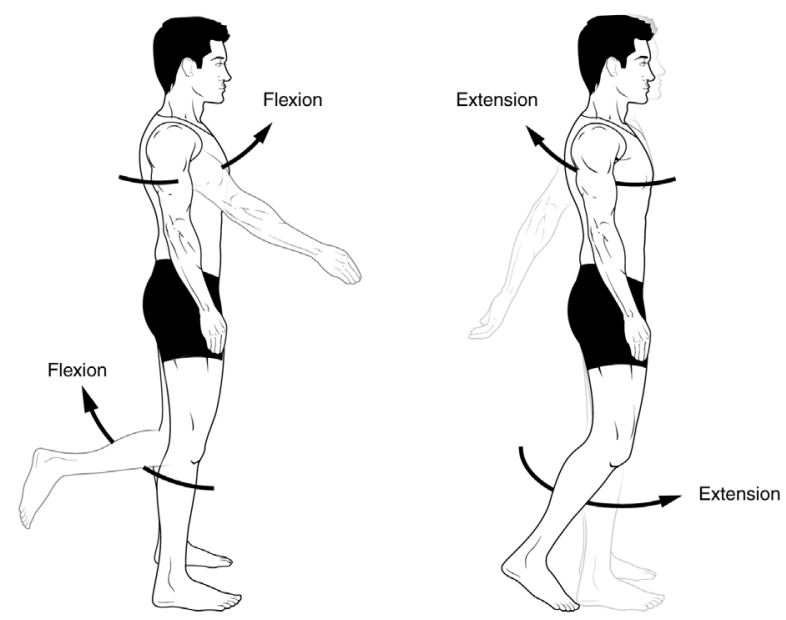

Figure 58. Angular movements: flexion and extension at the shoulder and knees. Adapted from [176].

As explained in the introduction, once the task is recorded by the MoCap system is necessary to evaluate the movements to identify the seriousness of the angles obtained. Because RULA and REBA are the most well-known methods for rapid assessment of musculoskeletal risks [42], their principles are going to be used to evaluate the movements in this experiment. 
REBA and RULA stipulates different levels of risk according to the displacement of arm and forearm from the neutral position [177], [178]. According to these postural analysis methods, the arm flexion and arm extension should maintain the position between $0^{\circ}$ and $20^{\circ}$ for a suitable work-position, the forearm flexion between $60^{\circ}$ and $100^{\circ}$, and the abduction of the arm should be avoided, every abduction-adduction increase the risk of musculoskeletal disorders (Figure 59).
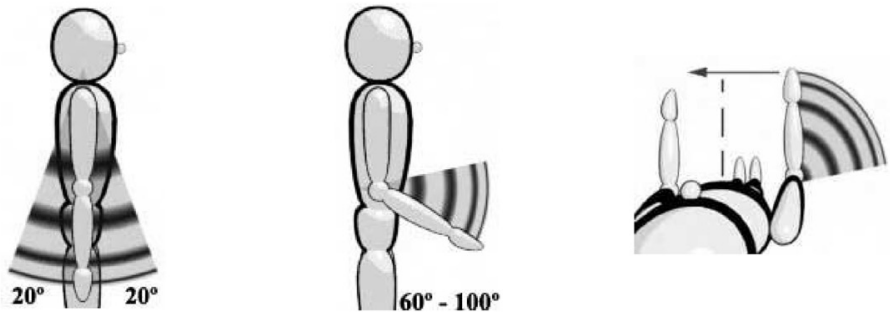

Figure 59. Ranges of arm flexion-extension, forearm flexion, and arm abduction-adduction according by REBA and RULA methods.

\subsection{Looking the appropriate MoCap system}

In order to found an appropriate system that accuracy registers the movements performed during laparoscopic procedures, three system were tested.

At the Institute for Medical Science and Technologies (IMSaT) in Dundee (Scotland, UK), they have access to a full VICON system and it give us the opportunity to test this system which is considered the gold standard of MoCap systems.

The experiment performed with this system consisted in record several surgeons performing a suture in a suturing pad (Figure 60). Over the pad were marked 11 dots that they had to follow in order to perform the suture as accurate as possible.

Just the movements were recorded, the accuracy of the suture was just a distraction to ensure the participants made the movements as similar as possible as the ones in the operating room. 


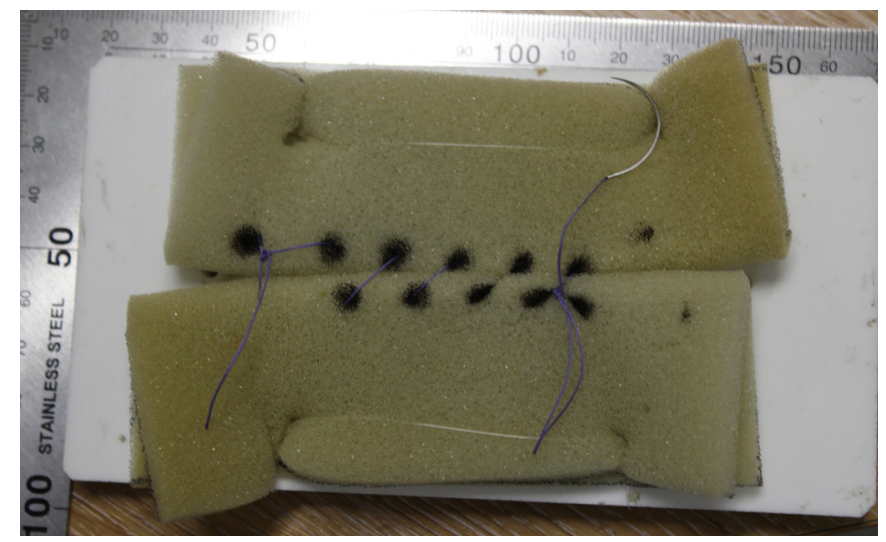

Figure 60. Suture pad used during the experiment at IMSaT laboratory (Dundee, Scotland).

The impressions with this system was good, but it showed some lacks, and the system seemed not to be the right choice for the purposed study. In order to obtain accurate results the system was quite appropriate, the main problem was the amount of cameras and markers that it requires (Figure 61). Another problem was that these cameras are really expensive (over $6500 €$ each one), and an accurate system requires at least 10 cameras to record a person's movements, doing the system really difficult to carry out to a different locations in order to evaluate surgeons in the simulation area or in the operating room.

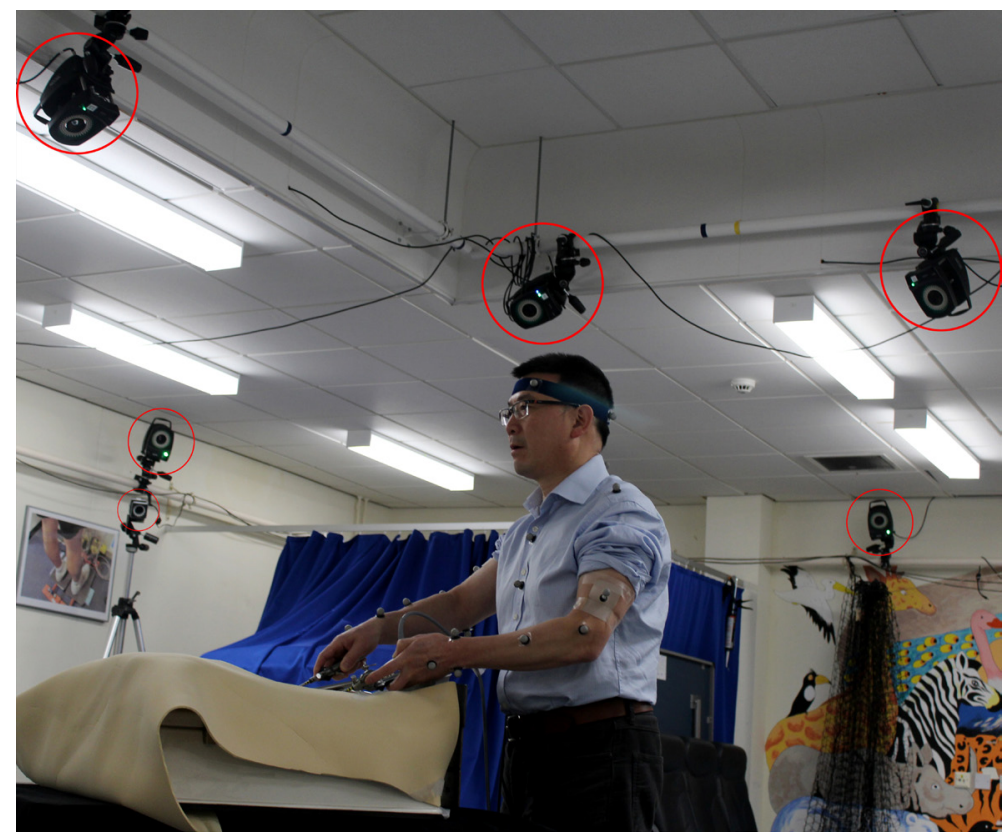

Figure 61. Laparoscopic surgeon being recorded by VICON system. Picture of the study performed at IMSaT laboratory in the Ninewells Hospital (Dundee, Scotland). Red circles mark only a fraction of the cameras used during this experiment. 
At the same time, The Kinect system was tested in order to evaluate the accuracy of the setup composed by two Kinect V2 sensors (Figure 62).

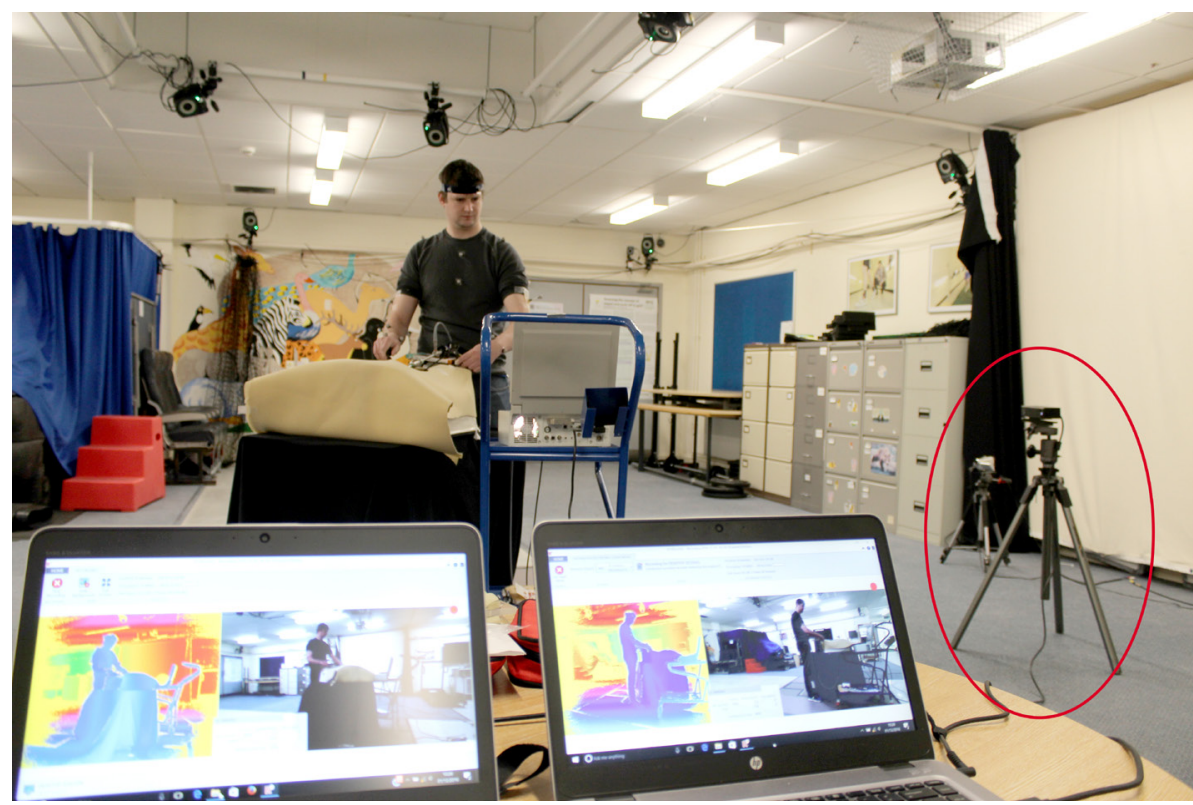

Figure 62.Testing the setup composed by two Kinect V2 sensors at IMSaT (Dundee, Scotland).

After a thorough literature review, it was found that sensors location used by C. Kim, J. Hong \& K. Chun [179] can be suitable for this experiment. With this setup, after several tests, it was found that the system was able to follow the movements more accurate when the surgeon location was the $90^{\circ}$ presented in Figure 63.

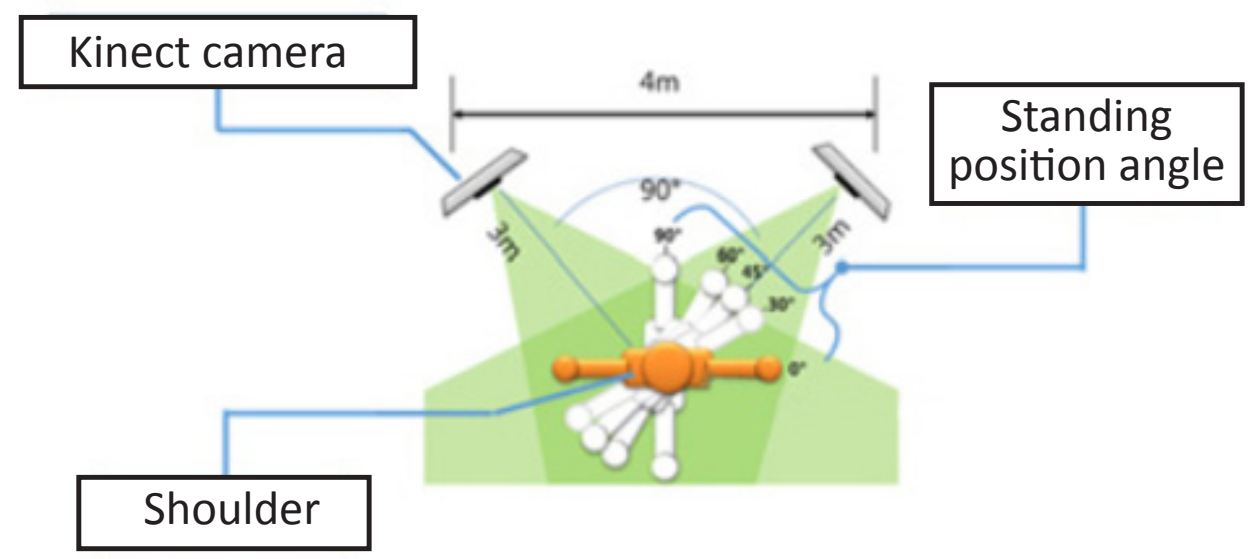

Figure 63. Setup for the tests performed by two Kinect V2. Adapted from [179]. 
Despite the economic benefits of this system, it was not suitable enough for this experiment. The main problem was that the dummy that follows the surgeon's movement, and had to proportion us the position of each section of the body, continually twisted its arms and it jeopardized the results. Relocate the dummy in the right position without affect the results of the experiment was really difficult.

In contrast, using the Tracker system, the video recording setup only requires conventional video cameras. Both cameras has to be located at the same distance and height in relation with the recording target. An orthogonal disposition for the cameras, one of those was located facing the surgeon and the other laterally recording the dominant arm. Surgeons were dressed in white, and black small balls were attached to their clothes in order to increase the colour contrast. It was used a clap in front of both cameras in order to synchronize both videos with the Tracker software.

To perform a successfully tracking from the videos once synchronized, was necessary to determine some parameters in order to say to the system what points it have to follow. In Figure 64 the three point of interest (shoulder, elbow and wrist) are identified by markers of different colors. These points were interesting for the study because allowed to evaluate the position of every join during the experiment and, more important, combine the data obtained from this positions to get the angular displacement performed by arm and forearm.

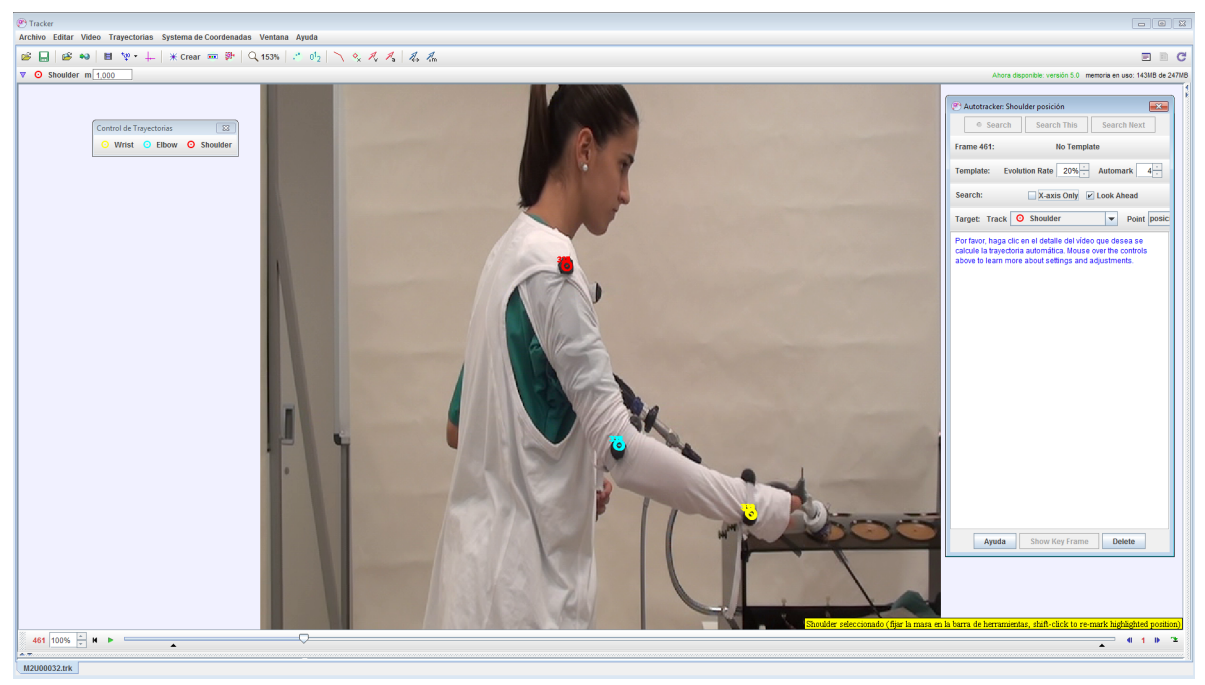

Figure 64. Positions to track with Tracker Vision System. The red point indicates the shoulder, the blue point indicates the elbow and the yellow point indicates the wrist. Screenshot from the test performed in the Area of Clinical Simulation (Hospital la Fe). 
To track the different points, is necessary to define reference measures, orientation, and the origin point to follow with the region in which the system has to search for the point in the next frame (Figure 65).

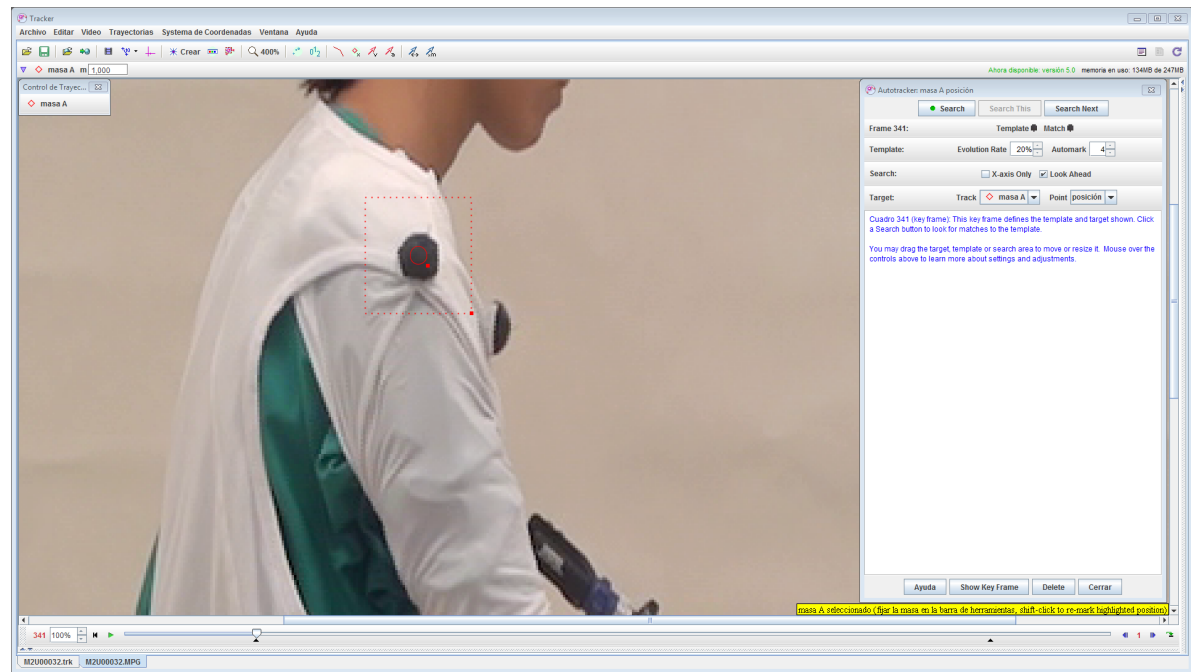

Figure 65. Point to follow and region to search in shoulder. Screenshot from the test with a participant in the study developed in the Area of Clinical Simulation (Hospital la Fe).

Once all these parameters are included in the evaluation is possible to run the software and track each point. Figure 66 shows a screenshot of the Tracker system tracking the wrist of a participant in a study performed in the Hospital la Fe of Valencia. In the picture the axis (purple) is linked to the elbow and follows its positions because is the point of reference to record the flexion-extension of the forearm. In the right side of the software is possible to see the outputs obtained in real time.

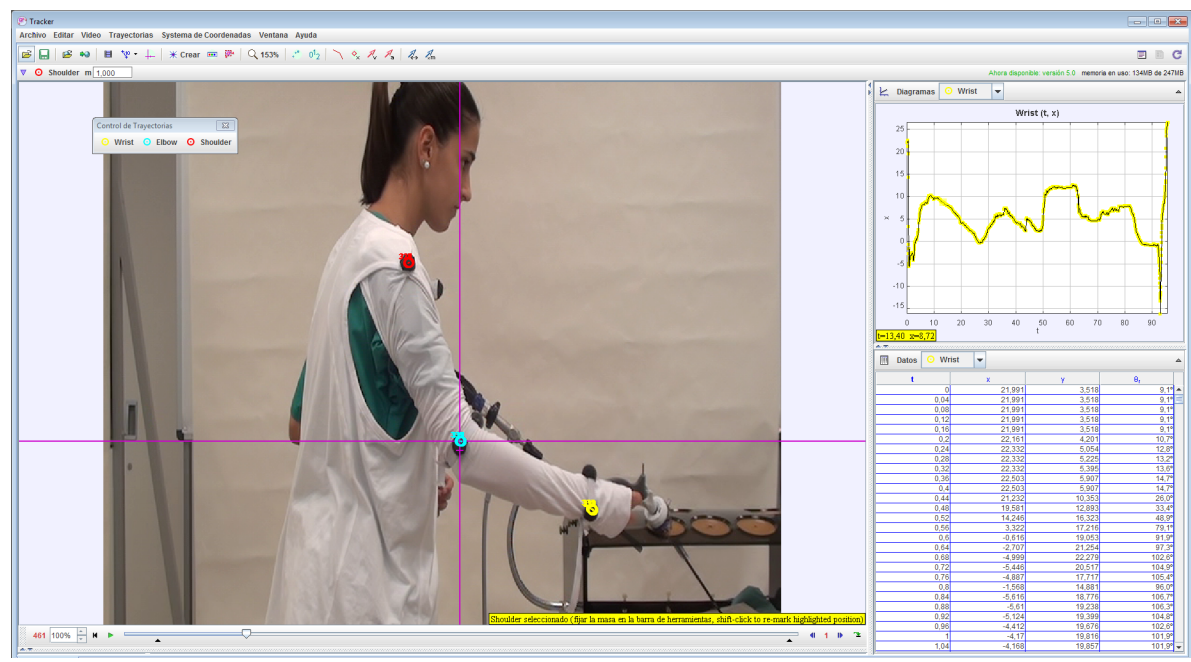

Figure 66. Tracker Vision System tracking the trajectory of the wrist. Axis point (purple) located in the elbow. Screenshot from the test with a participant in the study developed in the Area of Clinical Simulation (Hospital la Fe). 
Despite the basic appearance of the Tracker system, it has some benefits to be used during this experiment. In comparison with the VICON system, the Tracker is much cheaper and requires a simplest setup, which is really useful to carry out to the hospital in order to evaluate the surgeons.

In comparison with the KINECT system, the Tracker system provides more accurate results and is more flexible to modifications. The Tracker software perform the evaluation frame to frame, for this reason, if some of the points are not distinguish by the camera, it is easy to relocate the point or discard a specific frame in order to obtain valid results. It is true that it requires a huge amount of processing time and this processing has to be continually supervised by a human eye, but the system allows to stop the evaluation process and perform quick modifications in order to improve the accuracy of the tracking.

\section{Materials and methods 12.1. Participants}

This study employed 13 subjects, all of them right-handed, aged between 20 and 50, height between 153 and $184 \mathrm{~cm}$ (average $177 \mathrm{~cm}$ ), comprising surgeons and surgical residents of the Hospital La Fe, in Valencia (Spain), and external participants.

Six of these subjects had previous experience with laparoscopic instruments and were considered as experts because they could be influenced by their previous experience with conventional laparoscopic instruments. Seven participants had no prior experience, this participants were included in the experiment in order to identify the possible extrapolation of movements due to the training of the experienced surgeons.

Before the study, all the participants provided information about their previous experience in surgery and in the simulation area. 


\subsection{Instruments}

During the MoCap experiment, the instrument used (Figure 67) has the articulated handle-to-shaft angle attached to a conventional cylindrical handle.

Figure 67. PF prototype used during the MoCap experiment. The handle has a cylindrical shape in order to avoid the pain caused by the rings of the conventional pistol-grip handle.

The control instrument (Figure 68) used to the comparison on this studies is the $\operatorname{AdTec}^{\circledR}$ single use (B.Braun, Germany). This instrument presents the basic pistol grip configuration for the handle. The tip of the instrument was adapted with a marker tip in order to identify the path followed by the participants.

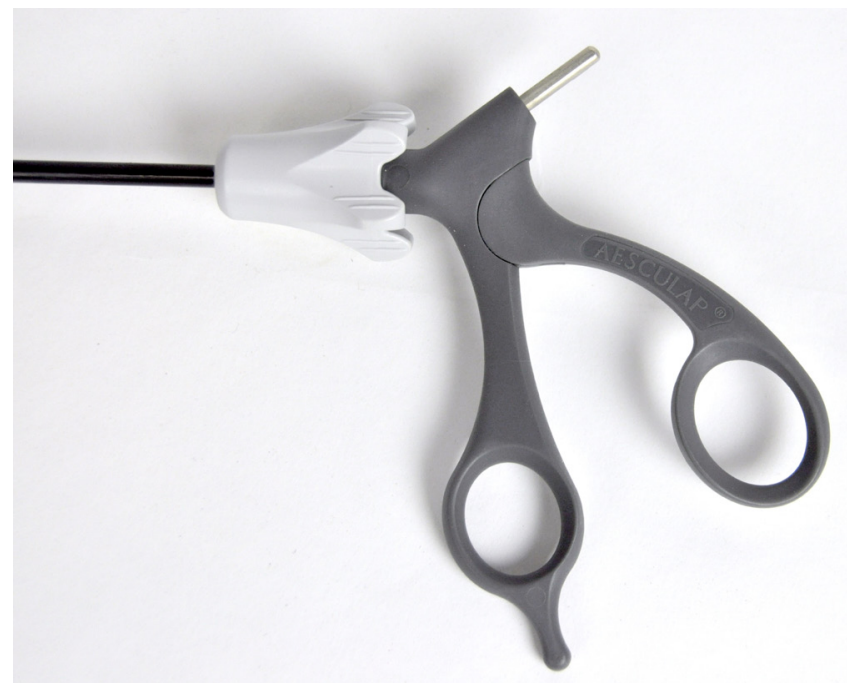

Figure 68. Control instrument used during the experiments. 
The experiment required a box-trainer (Figure 69) that was provided by the area of clinical simulation of the Hospital La Fe and a $5 \mathrm{~mm}$ trocar (Endopath XCEL ${ }^{\circledR}$ bladeless trocar, Ethicon, USA) in order to simulate the surgery conditions during the experiments.

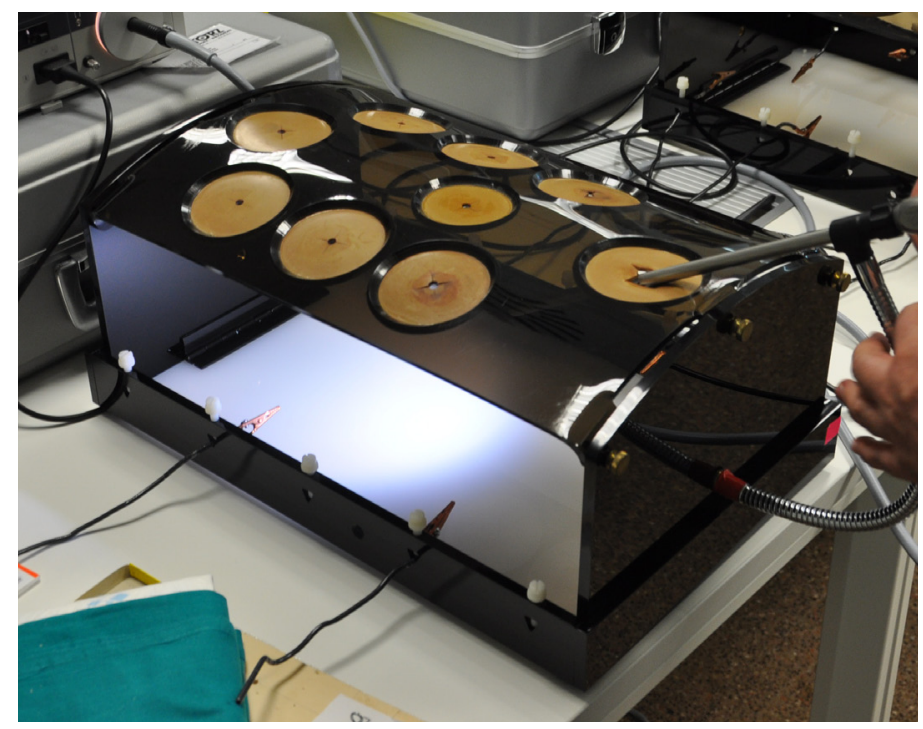

Figure 69. Box trainer model Szabo-Berci-Sackier (Karl Storz, Germany) used in the area of clinical simulation of the Hospital La Fe (Valencia, Spain).

\subsection{Protocol}

The MoCap study was a dynamic experiment used to evaluate the angles and displacements performed by the upper limbs during a laparoscopic simulation and compare the results between a control instrument and a PF prototype. The endpoint of the study was to identify the differences in the movements performed with the conventional pistol-grip instrument with fixed handle-to-shaft angle and the PF prototype used during this experiment.

The template used (Figure 70) is, as in the dynamic SEMG study, an adaptation of the test "Curve" used by Matern et al. in 2004 [144]. The three red dots were added as control points indicating extreme positions, the position of these three points were discussed with a group of surgeons in "La Fe" Hospital. The objective of these points was to obtain extreme values while trying to evaluate the more awkward surgical postures. 
This experiment was performed with non-functional instruments. Both instruments had ink based tips mounted, to enable users to mark the trajectory of their motion on the template.

The participant had to insert the laparoscopic instrument in the box-trainer by means of the trocar previously inserted in the box-trainer.

Once the tip was inside the box-trainer, the participant had to follow the curve test trying to not surpass the lines and maintain the tip of the instrument between both lines. These lines were located $2 \mathrm{~cm}$ to each other.

The path followed by the participant was painted over the template to give them the chance to recover the path when they leave it. After the circuit, the participants had to reach the three red dots, maintaining a static posture of 3 seconds in each one. Once the circuit is performed and the 3 dots reached, the participant had to extract the instrument of the box-trainer.

Before and after the experiment, the participants maintained a rest position in order to synchronize the video recorders. The participants received a detailed explanation of the protocol before the experiment to avoid stops once the experiment begun.

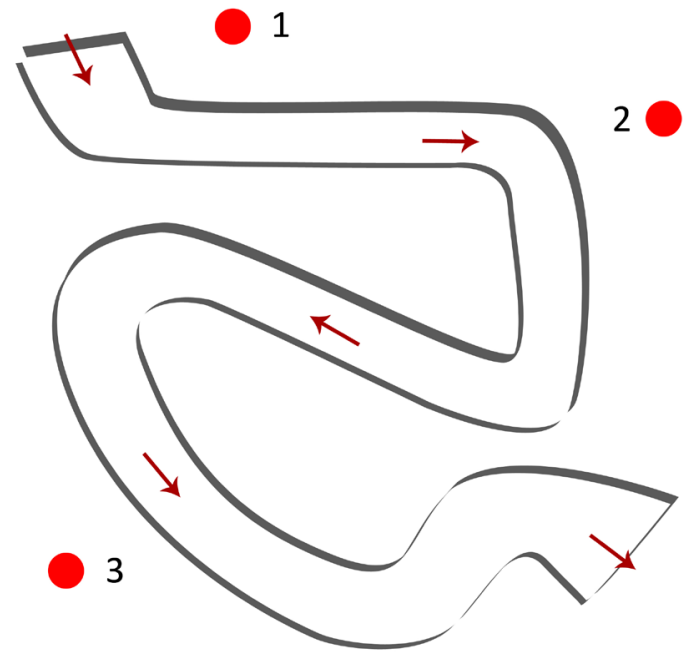

Figure 70. Curve test. The shape of the path was adapted from Matern et al. [144]. Red dots included to record some extreme positions, arrows indicate the direction of the test. 


\subsection{Data acquisition}

The MoCap record system consist in two cameras (SMX-C100RP, Samsung, South Korea) that were positioned at the front and the side of the participant. These captured the front and the side of each subject when they performed the task. The distance camera-participant was fixed at 2 meters, whereas the height of the cameras was adjusted for each participant to capture the movements of their arms throughout the experiment.

Each subject wore white clothes and two black ball markers were attached in each joint (wrist, elbow and shoulder). A white background was used to increase the contrast. This color contrast helped the software to more accurately track each ball during evaluation (Figure 71).

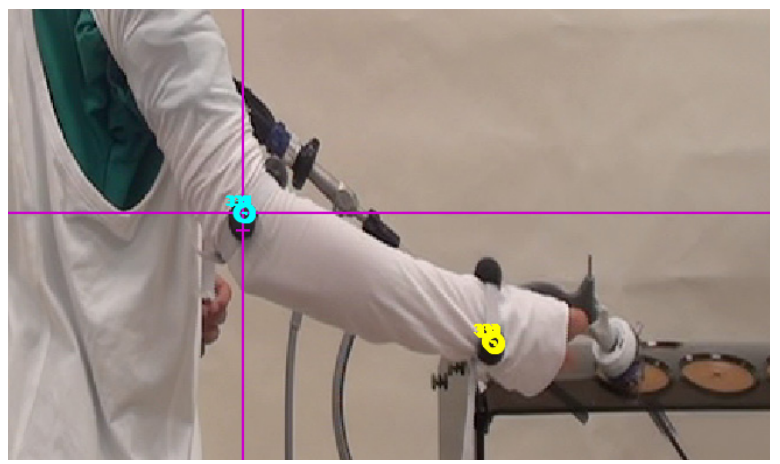

Figure 71. Tracking of elbow and wrist with the Tracker Video Analysis software.

The software used during the evaluations was "Tracker Video Analysis". This is an open source physics software that has the ability to follow pixels in the video recording allowing them to be tracked. This software has been validated before to evaluate laparoscopic tasks, obtaining accurate results [173].

This software provide us a list of positions $(X, Y)$ and angles of each point during the whole experiment. Each point had its own axis according to the movement evaluated. The axis for the wrist was located in the participant's elbow, and the axis for the elbow in the participant's shoulder. To avoid wrong data obtained from participants' movements, the whole system follows their movements 


\subsection{Data analysis}

As aforementioned, the movements evaluated during the MoCap experiment were categorized into three different groups: arm abduction-adduction, arm flexionextension and forearm flexion.

In order to measure the risk of the movements performed by each participant, REBA and RULA measures have been taken into account. The neutral posture for arm flexion and arm extension is between $0^{\circ}$ and $20^{\circ}$ and for forearm flexion is between $60^{\circ}$ and $100^{\circ}$. The arm abduction and arm adduction movements do not have any established range but a range similar to arm flexion-extension was used (neutral position $=0^{\circ}-20^{\circ}$ ) to evaluate the risk of abduction-adduction.

By means of the tracker software, each joint (wrist, elbow and shoulder) was analyzed 25 timers per second for the duration of each recording. The tracker software provides results of positions and angles displacements. The results obtained were statistically analyzed using the unpaired t test with Welch's correction because the groups did not present equal standard deviations.

\section{Results}

The collected data were organized according to each movement evaluated: arm abduction, arm adduction, arm flexion, arm extension and forearm flexion.

Figures 72 to 77 show the distribution of the values of each movement, the bars indicate the maximum and minimum value reached by each movement and the box the interval from the percentile 25 to percentile 75 . 


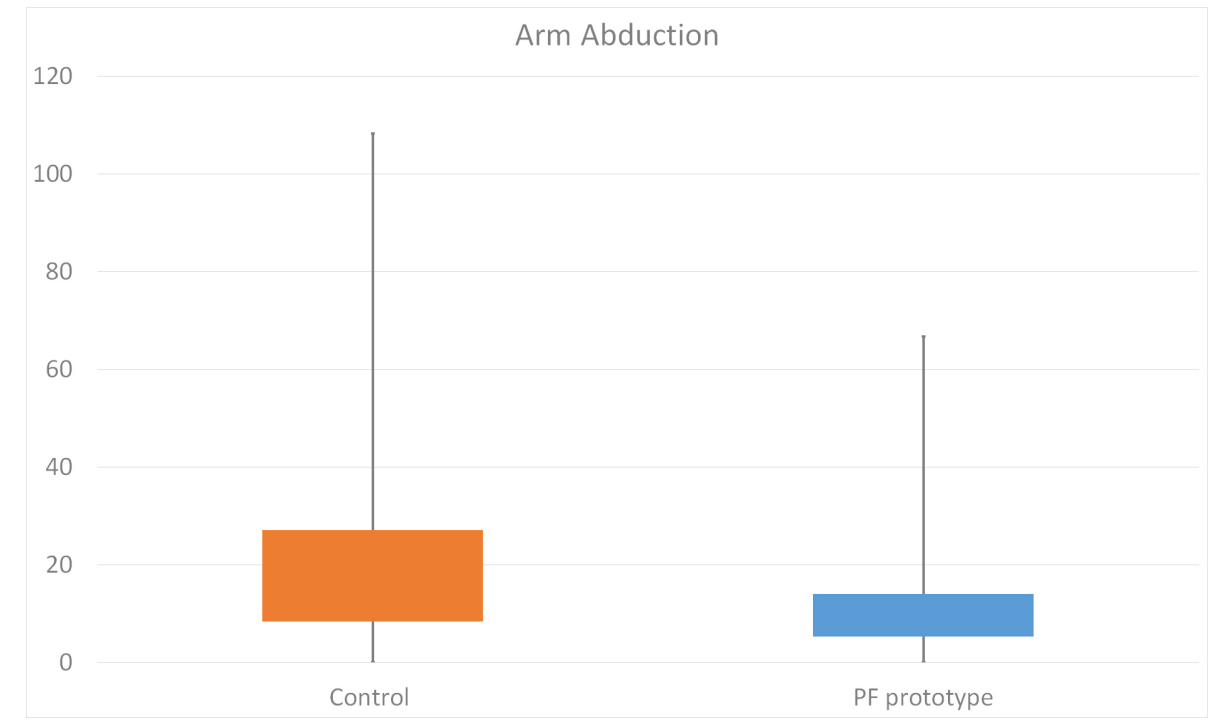

Figure 72. Arm abduction values by instrument. Boxes indicate the main amount of values (percentiles 25 to 75 ). The error bars indicate the maximum and minimum value reached.

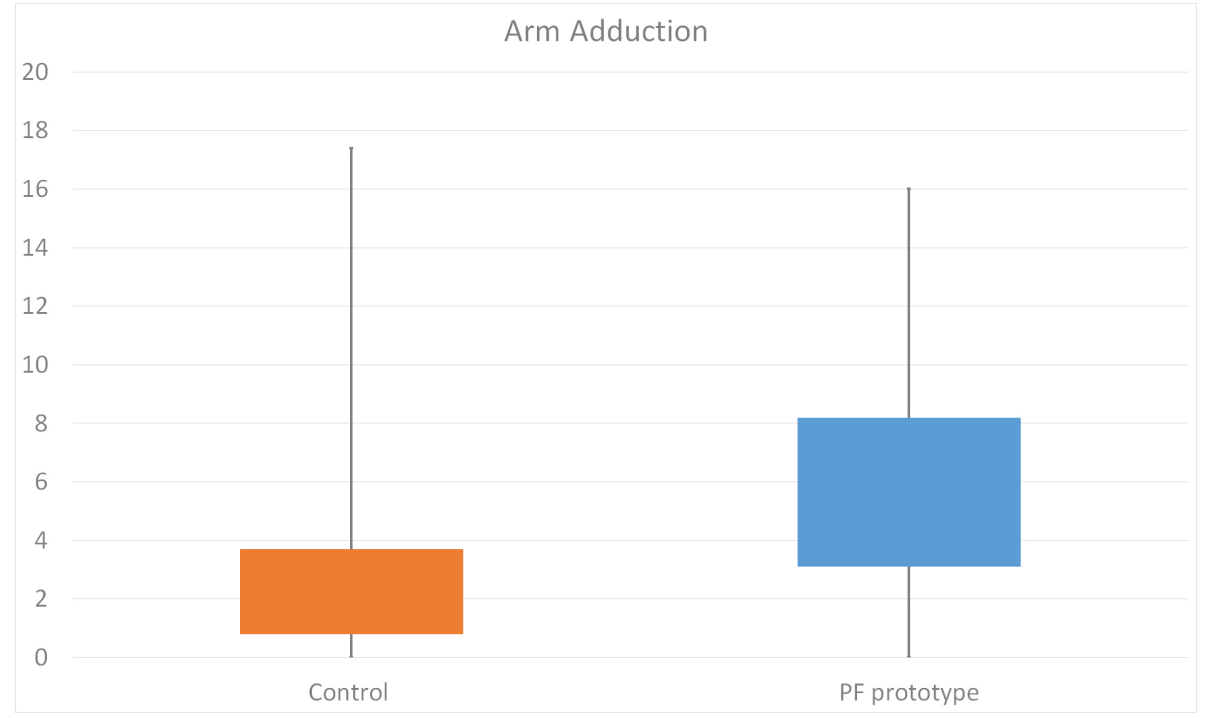

Figure 73. Arm adduction values by instrument. Boxes indicate the main amount of values (percentiles 25 to 75 ). The error bars indicate the maximum and minimum value reached. 


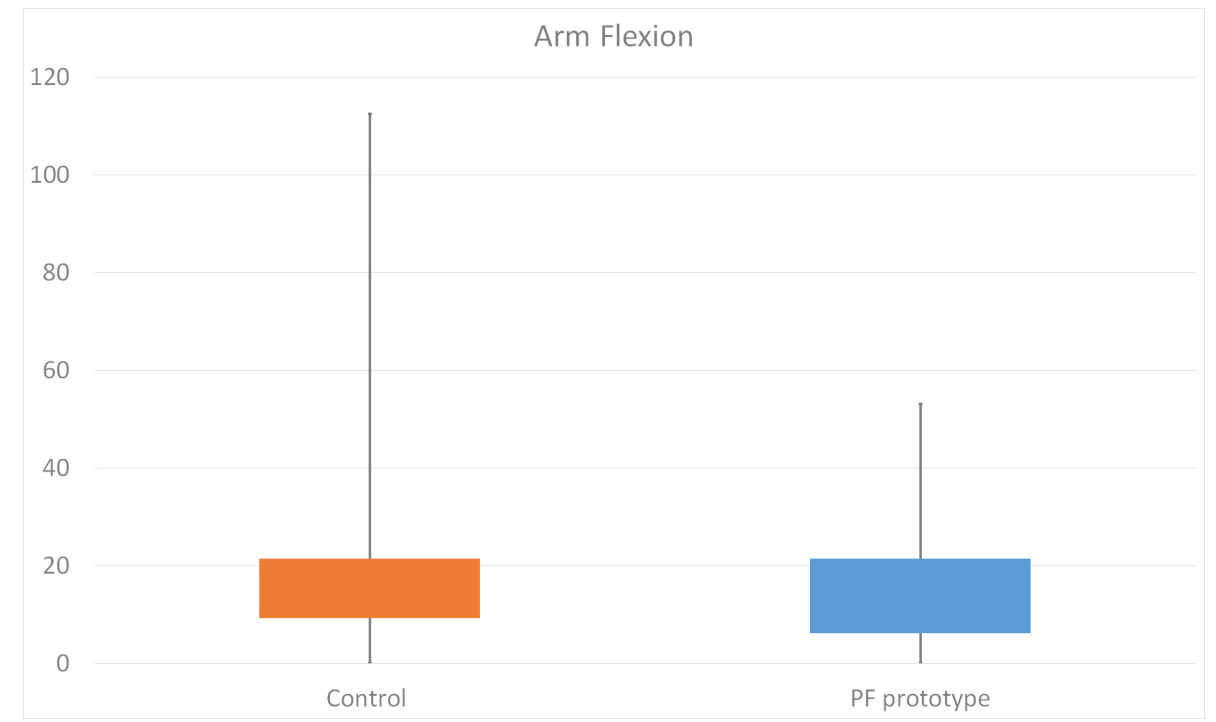

Figure 74. Arm flexion values by instrument. Boxes indicate the main amount of values (percentiles 25 to 75). The error bars indicate the maximum and minimum value reached.

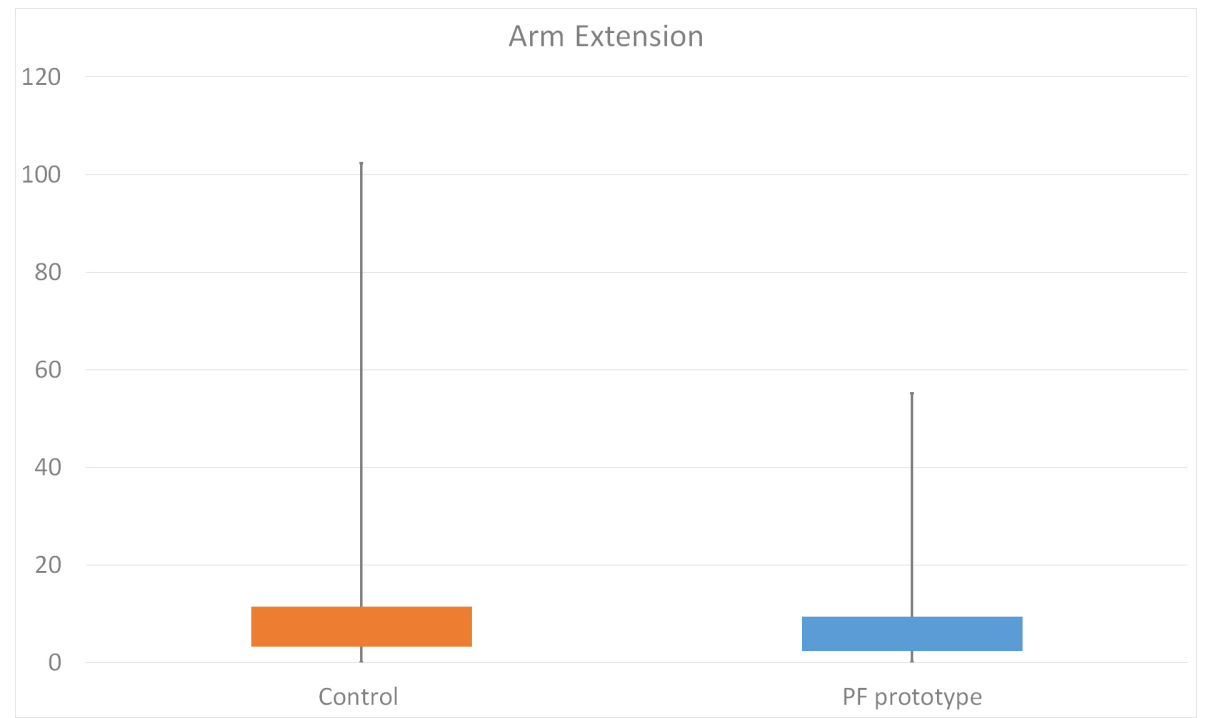

Figure 75. Arm extension values by instrument. Boxes indicate the main amount of values (percentiles 25 to 75 ). The error bars indicate the maximum and minimum value reached. 


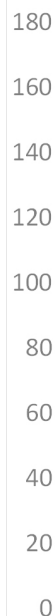

Figure 76. Forearm flexion values by instrument. Boxes indicate the main amount of values (percentiles 25 to 75). The error bars indicate the maximum and minimum value reached.

The amount of time that participants keep their movements in the range of neutral positions are presented in Table 5. The "neutral" column on this table indicate the range considered neutral position for each movement.

Table 5. Percentage of neutral position by each movement with both instruments.

\begin{tabular}{|c|c|c|c|}
\hline & Neutral & Control & PF prototype \\
\hline Arm Abduction & $0^{\circ}-20^{\circ}$ & $61,4 \%$ & $91,7 \%$ \\
\hline Arm Adduction & $0^{\circ}-20^{\circ}$ & $100 \%$ & $100 \%$ \\
\hline Arm Flexion & $0^{\circ}-20^{\circ}$ & $68,3 \%$ & $71,5 \%$ \\
\hline Arm Extension & $0^{\circ}-20^{\circ}$ & $92,5 \%$ & $96,7 \%$ \\
\hline Forearm Flexion & $60^{\circ}-100^{\circ}$ & $53,9 \%$ & $62,8 \%$ \\
\hline
\end{tabular}

In order to identify differences between experts and participants without previous experience, Table 6 shows values separated in both groups. The results indicate the average angle in each position for both groups with the PF prototype and with the control instrument, the maximum values reached during the experiment and the difference between means of both instruments.

The results indicate that the biggest differences for subjects with experience were presented in arm abduction $(7,18 \pm 0,16)$, arm adduction $(4,08 \pm 0,05)$ and forearm flexion $(13,65 \pm 0,27)$. In the case of subjects without previous experience, the biggest differences were presented in arm abduction $(13,53 \pm 0,18)$, arm flexion $(8,83 \pm 0,25)$ and forearm flexion $(8,91 \pm 0,28)$. 
Table 6. Mean, maximum and difference of the movements performed with each instrument by experts and non-experts.

\begin{tabular}{|c|c|c|c|c|c|c|}
\hline & & Instrument & Mean & Max & Difference & $\rho^{*}$ \\
\hline \multirow{4}{*}{ 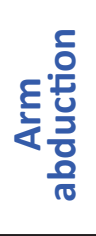 } & \multirow{2}{*}{ Experts } & Prototype & $11^{\circ}$ & $66,7^{\circ}$ & \multirow{2}{*}{$7,18 \pm 0,16$} & \multirow{2}{*}{$<0,05$} \\
\hline & & Control & $18,18^{\circ}$ & $108,3^{\circ}$ & & \\
\hline & \multirow{2}{*}{ No experts } & Prototype & $9,42^{\circ}$ & $41,4^{\circ}$ & \multirow{2}{*}{$13,53 \pm 0,18$} & \multirow{2}{*}{$<0,05$} \\
\hline & & Control & $22,96^{\circ}$ & $101,5^{\circ}$ & & \\
\hline \multirow{4}{*}{ 튼 $\frac{\text { 든 }}{\frac{0}{2}}$} & \multirow{2}{*}{ Experts } & Prototype & $5,97^{\circ}$ & $16^{\circ}$ & \multirow{2}{*}{$4,08 \pm 0,05$} & \multirow{2}{*}{$<0,05$} \\
\hline & & Control & $1,89^{\circ}$ & $5,5^{\circ}$ & & \\
\hline & \multirow{2}{*}{ No experts } & Prototype & $3,14^{\circ}$ & $9,2^{\circ}$ & \multirow{2}{*}{$5,62 \pm 0,29$} & \multirow{2}{*}{$<0,05$} \\
\hline & & Control & $8,76^{\circ}$ & $17,4^{\circ}$ & & \\
\hline \multirow{4}{*}{$\begin{array}{l}\frac{c}{ㅇ} \\
\frac{0}{x} \\
\frac{0}{6} \\
\frac{\varepsilon}{2} \\
\frac{1}{4}\end{array}$} & \multirow{2}{*}{ Experts } & Prototype & $15,73^{\circ}$ & $53,1^{\circ}$ & \multirow{2}{*}{$1,16 \pm 0,12$} & \multirow{2}{*}{$<0,05$} \\
\hline & & Control & $16,89^{\circ}$ & $84,4^{\circ}$ & & \\
\hline & \multirow{2}{*}{ No experts } & Prototype & $11,44^{\circ}$ & $47,3^{\circ}$ & \multirow{2}{*}{$8,83 \pm 0,25$} & \multirow{2}{*}{$<0,05$} \\
\hline & & Control & $20,27^{\circ}$ & $112,5^{\circ}$ & & \\
\hline \multirow{4}{*}{$\frac{\varepsilon}{\frac{c}{2}} \frac{\frac{c}{0}}{\frac{c}{d}}$} & \multirow{2}{*}{ Experts } & Prototype & $6,05^{\circ}$ & $55,1^{\circ}$ & \multirow{2}{*}{$1,24 \pm 0,21$} & \multirow{2}{*}{$<0,05$} \\
\hline & & Control & $7,29^{\circ}$ & $53,8^{\circ}$ & & \\
\hline & \multirow{2}{*}{ No experts } & Prototype & $7,01^{\circ}$ & $25,8^{\circ}$ & \multirow{2}{*}{$2,01 \pm 0,15$} & \multirow{2}{*}{$<0,05$} \\
\hline & & Control & $9,02^{\circ}$ & $102,3^{\circ}$ & & \\
\hline \multirow{4}{*}{ 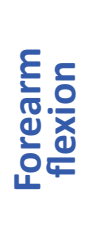 } & \multirow{2}{*}{ Experts } & Prototype & $86,14^{\circ}$ & $143,9^{\circ}$ & \multirow{2}{*}{$13,65 \pm 0,27$} & \multirow{2}{*}{$<0,05$} \\
\hline & & Control & $72,49^{\circ}$ & $143,2^{\circ}$ & & \\
\hline & \multirow{2}{*}{ No experts } & Prototype & $72,86^{\circ}$ & $155,3^{\circ}$ & \multirow{2}{*}{$8,91 \pm 0,28$} & \multirow{2}{*}{$<0,05$} \\
\hline & & Control & $63,94^{\circ}$ & $148,6^{\circ}$ & & \\
\hline
\end{tabular}

\section{Discussion}

The main goal of this study was to identify and evaluate the effect of the PF in the participants' movements during a laparoscopic simulation. In general, the movements performed by the PF prototype were closer to the optimal position according to REBA [177] and RULA [178] methods than the movements with the control instrument. Despite arm abduction and arm adduction are not categorized by these methods, both methods indicate that abduction and adduction increase the risk of musculoskeletal injuries and these movements should be as near as possible to $0^{\circ}$. 
The results showed that the PF prototype increased the amount of time in neutral position during the experiment, especially in arm abduction, where the participants keep their arm position in neutral ranges $91,7 \%$ of the time (Table 5 ). This is an interesting results because the arm abduction movement is considered critic in laparoscopic surgery [19], [21], [37], [75], [76] and difficult to improve because of the practice [151]. The results obtained with the control instrument in the simulation task are consistent with the arm abduction results presented by previous literature during real procedures [76] being the results in real practice slightly worse probably due to the tension inherent to a real procedure.

Arm abduction (Figure 72), Arm flexion (Figure 74), and arm extension (Figure 75) movements were improved by the participants while using the PF prototype, keeping the majority of their movements inside the range of neutral positions (arm abduction 91,7\%; arm flexion $71,5 \%$; arm extension $96,7 \%$ ) and their maximum displacements nearest to these ranges than the control instrument.

The results also indicate that, despite arm adduction and forearm flexion maintain or increase the time in neutral position in comparison with the control instrument (Table 5), the PF prototype require more arm adduction than the control instrument (Figure 73), and the forearm flexion reach higher maximum values than the control instrument (Figure 76)

The information was used to identify differences on the movement performed by expert participants and novices too, in order to see if previous knowledge increase or reduce the PF benefits. Previous observational sessions indicate us that the participants with previous knowledge tries to replicate the movements learned with the new instrument, hindering the adaptation of the new movements allowed by the prototype instrument. Surgeons spend a huge amount of time training in the simulation area before their first laparoscopic procedures in the operation room. Is not weird to think that, after all the training period, surgeons acquire some habits and postures because of the conventional instruments. And those habits are difficult to avoid with different instrumentation. 
But, despite this movement extrapolation by participants with experience, the results show that both groups would improve their ergonomic position with the PF prototype. According to the results presented in Table 6, with the PF prototype, non-experts presented an average arm abduction of $9,42^{\circ}$, which means an average reduction of $13,53^{\circ}$ respect to the values obtained with the control instrument $\left(22,96^{\circ}\right)$. Participants with previous experience also reduced their arm abduction with the PF prototype, in this case, the reduction was in $7,18^{\circ}$. With the PF prototype, user without experience also found this reductions in arm adduction $\left(5,62^{\circ}\right)$, arm flexion $\left(8,83^{\circ}\right)$, arm extension $\left(2,01^{\circ}\right)$ and forearm flexion $\left(8,91^{\circ}\right)$. Participants with experience found this reductions also in arm flexion $\left(1,16^{\circ}\right)$ and forearm flexion $\left(1,37^{\circ}\right.$ considering $80^{\circ}$ the optimal position for this movement)

Despite, participants without experience presented higher differences between control instrument and PF prototype, it could be wrongly attributed to an elevated improvement due to the PF prototype. It is important to clarify that the participants without experience presented worse values than experienced participant with the control instrument, which suggests that the control instrument forces the participant to maintain uncomfortable positions but the experienced surgeons, with trainee, can improve their position.

The general results indicate that the concept of PF provides positions closer to the neutral range than the control instrument. And even closer in the first stages of laparoscopic training. The non-experienced subjects achieve the best results in the test and higher differences between the control and the prototype instrument.

This study supports the idea that the implementation of a PF elements could be helpful to reduce the upper limb extreme displacements during laparoscopic practices. This improvement should be taken into account for developing future instrument designs. 



\section{Chapter 5.}

Design and prototype of a PF instrument for laparoscopic surgery. User-centered design. 


\section{Introduction. The user- centered design}

The design process used to design the instrument presented on this manuscript was the "user-centered design". Despite this term is widely known as a design processes in which end-users influence how a design takes shape it is much more, it is considered both a broad philosophy and variety of methods.

In the creation process, there is a spectrum of ways in which users are involved in user-centered design but the important concept is that users are involved one way or another [180]. For examples, some types of user-centered design consult users about their needs and involve them at specific times during the design process; typically during requirements gathering and usability testing. At the opposite end of the spectrum there are user-centered design methods in which users have a deep impact on the design by being involved as partners with designers throughout the design process [180]. Carrying out a task analysis as well as a general requirements analysis, carrying out early testing and evaluation, and designing iteratively makes designers to be focused on the user's needs [181]. The term "user-centered design" was coined by Donald Norman in the 1986, on his research laboratory in the University of California San Diego [180]. Latterly, his book The Design of Everyday Things [182] becomes one of the basics lectures on this field.

There is a related movement, Human-Centered Design, which expanded the focus from the user in interaction with the system to considering how human capabilities and characteristics are affected by the system beyond direct interaction with the interface or system itself. According to IDEO, one of the most important design studios worldwide which base its work on this approach, Human-Centered Design "is a process that starts with the people you're designing for and ends with new solutions that are tailor-made to suit their needs".

Accordance with this approach, humans should be seen as the most important element of information systems. The use of the Human-Centered approach surfaces in three ways [181]: 
- First, consideration is given to the fact that the introduction of a new system engenders changes in the organization of peoples' behaviors and activities. These behavioral changes also affect others. So, user needs and demands, situational effects, and technological requirements are considered in tandem.

- Second, human-centered design addresses the fact that more and more systems are being built where users do not interact directly with the technology as "users." For example, bed sensors which are programmed to track automatically when a person gets out of bed and to raise an alarm if they are not back in bed within a programmed time limit.

- Finally, Human-Centered Design tends to look to the longer-term effects, as well as the immediate, task-related issues that occur at human-system "touch point" moments.

This work uses this approach because it has evident benefits for the final users and because technologies must be demonstrated as not being error prone [183], especially in areas as medicine. Unfortunately, a number of cases have been documented that show that medical devices, as infusions pumps [184], handheld e-prescribing application [185], CPOE (Computerized Physician Order Entry) [186] are in fact error prone [187]. The use of User-centered (and human-centered) design methods, tend to emphasize user participation in the design process for ideation and evaluation of design options [181], the solutions obtained among this approach guarantee good products and avoid failures, ensuring that product do work and people can use them [188].

\subsection{User-centered methodology}

There are multiples principles that underlie user-centered methodologies. This design approach is based upon an explicit understanding of users, tasks, and environments; is driven and refined by user-centered evaluation; and addresses the whole user experience. The following are the general phases of the User-Centered methodology [189]: 
- Specify the context of use: Identify the people who will use the product, what they will use it for, and under what conditions they will use it.

- Specify requirements: Identify all business requirements or user goals that must be met for the product to be successful.

- Create design solutions: This part of the process may be done in stages, building from a rough concept to a complete design.

- Evaluate designs: Evaluation, ideally through usability testing with actual users.

The specific context of use and requirements are been widely tackled in the first chapter of this manuscript. On the following chapter, the last two phases of the methodology will be tackled: create a design solution and evaluate it with real users.

\section{Creating the design solution. Evotool}

\subsection{Design of a PF hand-held laparoscopic instrument}

As explained above, around $80 \%$ of the surgeons report physical symptoms or discomfort as a direct result of the laparoscopic practice [58]-[60]. Upper limbs reach frequent awkward postures, as excessive shoulder abduction or wrist flexion Figure 77), affected by a fixed access port and the instrument design [6], [21], [68]. 


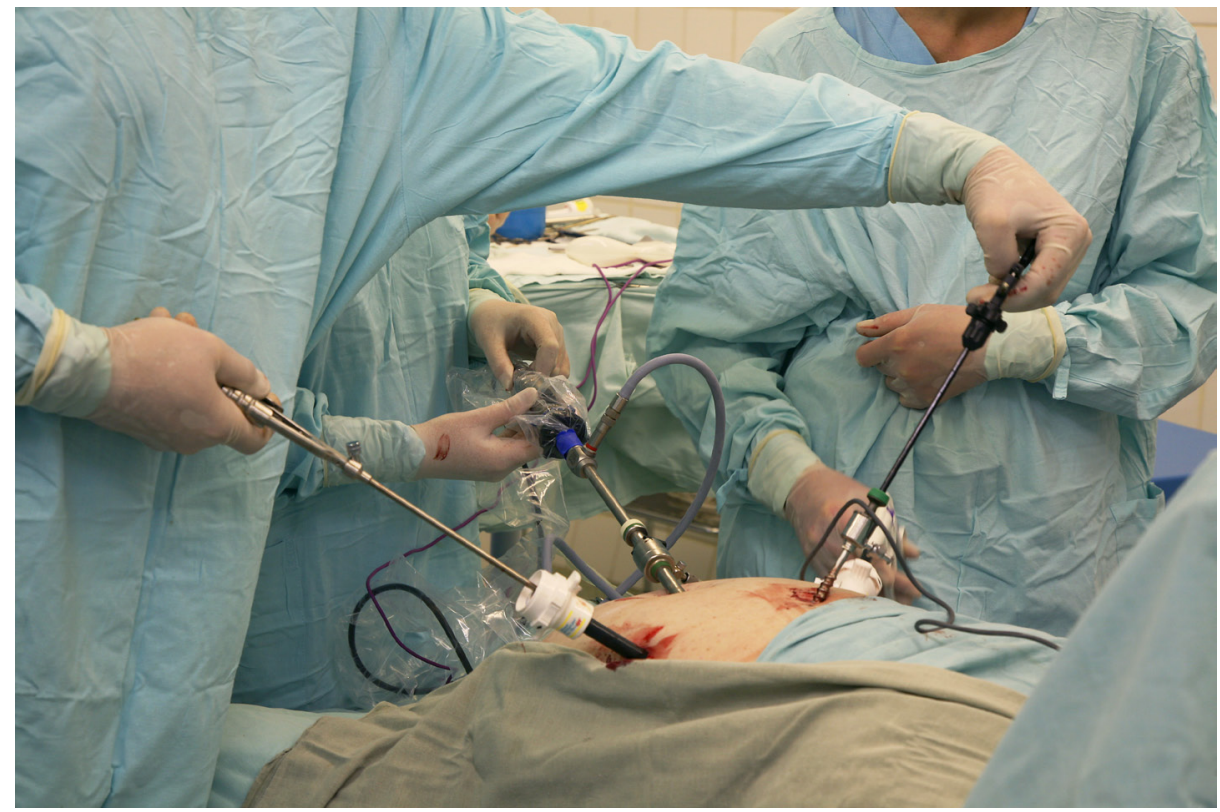

Figure 77. Laparoscopic surgeon performing an intervention. In the picture is easily recognizable the excessive arm abduction and wrist flexion to perform the procedure.

In order to reduce the negative consequences that this procedures has in the surgeons' body a new instrument was designed. The hypothesis used to set up this instrument design as well as all the studies and work included in this manuscript was explained in the justification section as follow "if you allow people to work without restrictions in laparoscopic surgery, they would adopt more beneficial positions for their musculoskeletal system being their movements and postures closer to neutral positions".

Once the hypothesis was confirmed with the MoCap and sEMG experiment was the moment to implement the PF in a usable prototype in order to receive feedback of the final users.

The literature about ergonomic laparoscopic instruments is mainly focused in the improvement of pistol-grip handle (see "Requirements for an ergonomic laparoscopic handle"). A solution using these criteria is not going to really improve the current situation, because the configuration of this handles cause a lot of problems of different kind. The purpose of this work is not only to design a simple handle with an articulated element attached to one of the sides. The final goal is to design an instrument that accomplish some ergonomic expectations. As explained in the introduction section "The design of ergonomic laparoscopic hand-held instrument", the surgeon should choose an instrument with the next characteristics [61]: 
- It has to enable the surgeon to keep both wrists in a neutral position.

- It has to allow the surgeon to keep both arms at the sides of their body.

- The handle has to avoid pressure points on the hands.

- It has to allow the surgeon to apply force with a power grip hand position.

- It has to allow fine manipulation with a precision grip hand position.

With these basic guidelines different design concepts were created, considering suggestions from Francisco Dolz, surgeon and chief of the Simulation area of the Hospital La Fe and one of the members of this project. Some of this design concepts were taken to the prototyping phase in order to be evaluated again.

\subsection{Prototyping}

A prototype is, generally speaking, a preliminary model designed to guide the development of a complex project. Prototypes are categorized according to its level of fidelity.

The prototype fidelity is considered to be the resolution (the refinement and detail) of the model [190]. Until 1996, the theoretical models that addressed the fidelity in usability testing were concentrated on the fidelity of the technical system, without taking consideration of issues such users characteristics and the testing environment [191].

Nevertheless, in 1996 Virzi et al. [192] proposes a model with four dimensions to evaluate the fidelity during a usability test:

- Breath of features refers to the number of features the prototype supports.

- Each of these features can then vary in its degree of functionality, or the extent to which the details of its operation are complete. 
- Similarity of interaction refers to how one communicates with the product (whether by pressing buttons, clicking a mouse, touching a screen, speaking, etc.).

- Aesthetic refinement refers to aspects of the product that do not directly influence its functionality, such as choice of colours and graphic design.

The range of prototyping methodologies are generally described within a spectrum of fidelity from low-fidelity to high-fidelity [193], although prototype fidelity is difficult to define precisely, a prototype that compromises on one or more of these four dimensions in a way that is obvious to the user is a low fidelity prototype [192]. The term mid-fidelity used to describe prototypes which are neither low- nor highfidelity and therefore lie somewhere in the middle along that axis [193].

In web usability testing for example, low fidelity prototype is only a paper prototype that identify each element of the web that contrary to high fidelity prototype that is an interactive visual basic system that allows to test the functionality of the product. In this case, the majority of the studies concluded that the reduced fidelity prototypes provided equivalent results to fully operational products [66].

Nevertheless, the decision of selecting a prototype for physical products entails a dilemma. A prototype of too high fidelity is very time-consuming and expensive to build. But the findings obtained with a prototype of too low fidelity may not be valid. This requires the careful consideration of what level of fidelity would be more adequate for the test [66].

Low fidelity prototypes may have fairly complete breadth of features and degree of functionality and so many be similar to the final product on these dimensions, but users do not typically interact with this prototypes in the same manner as the final product, and they do not typically look and feel the same with respect to the last dimension, aesthetic refinement [192]. The use of this prototypes (Figure 78) allowed to obtain quick answers to different questions during the development process avoiding the high costs and time required by high-fidelity prototypes. 


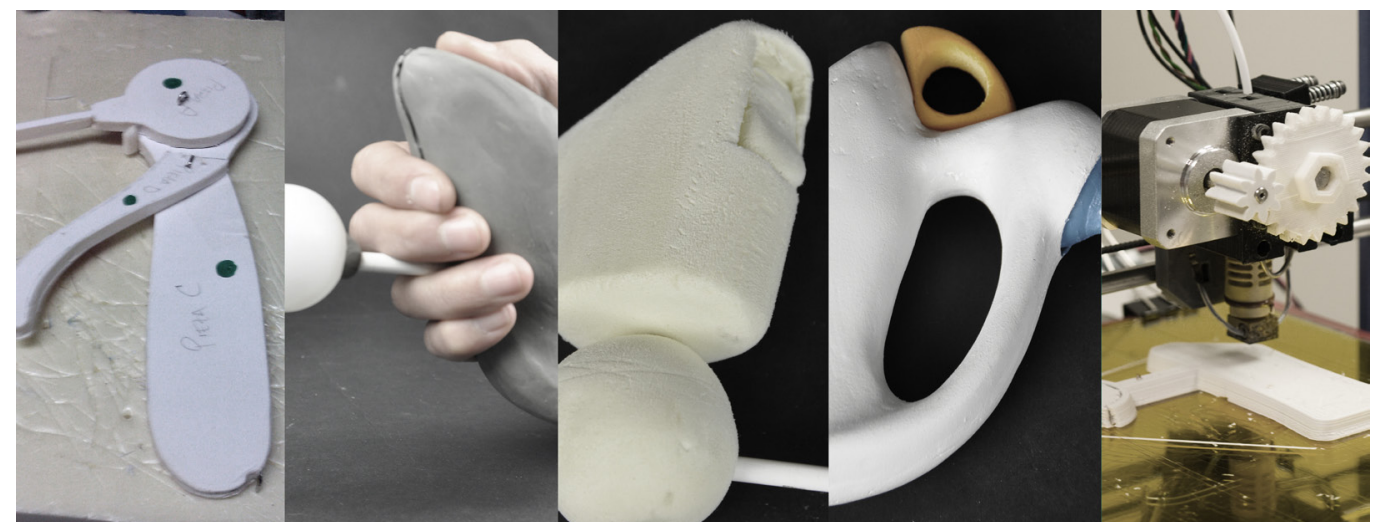

Figure 78. Some of the low fidelity prototypes in different steps of the process developed during this thesis.

All the users' opinions about the different design guided the research towards a design that could be considered as a new section in the surgeons arm, it has similar functions as the elbow in the arm but with the possibility to be continually turned around (Figure 79). With this idea, the surgeons would increase their PF and the awkward positions would be bearing by the instrument instead from the surgeons.

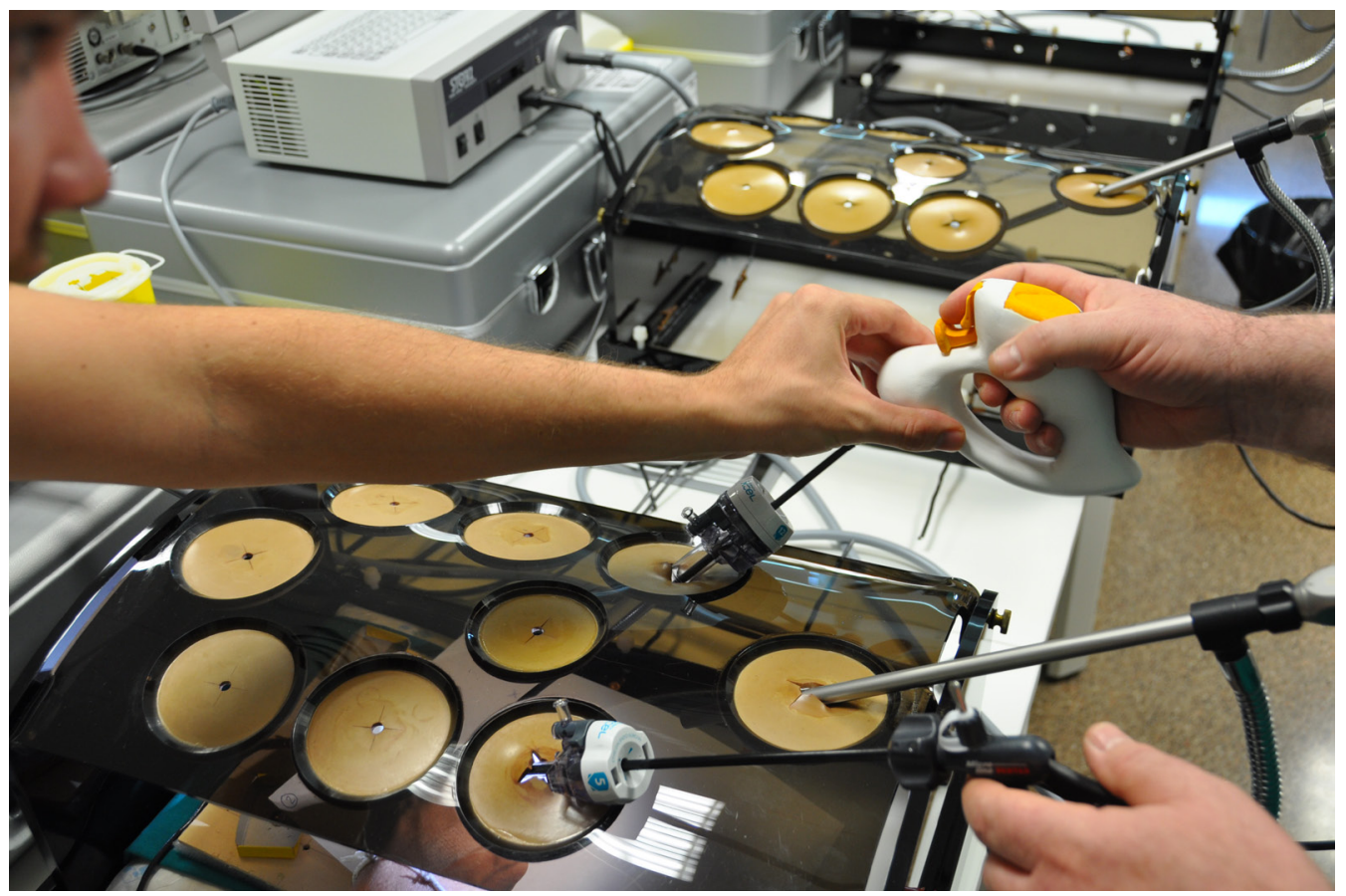

Figure 79. Low fidelity prototype evaluation in Hospital La Fe of Valencia (Spain). 


\subsection{Evotool}

The design proposed was called "Evotool" and was patented in 2017 by the Universitat Politècnica de València" (ES2611601). Evotool presents an inner sphere attached to the shaft that forms a ball-joint articulation with the handle and allows it to rotate with respect to each other (Figure 80). The inner sphere attached to the shaft, makes possible the surgeon to vary the working angle of the instrument, avoiding uncomfortable positions.

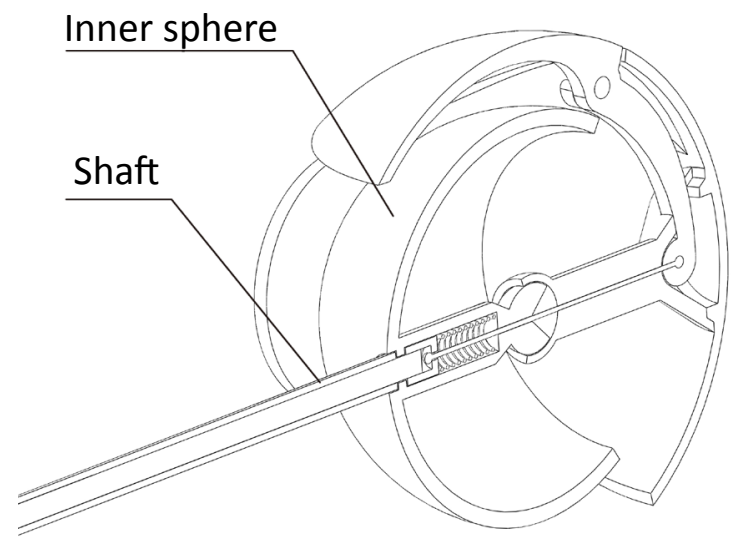

Figure 80 . Sagittal plane section, $45^{\circ}$ view. PF prototype (Evotool).

The trigger controls the movements and strength of the distal tip to grasp, cut, or suture, and the blockage system allows to rotate the distal tip and reach the right position (Figure 81). Once the distal tip is oriented, the surgeon can recover the rest position and continue with the procedure.
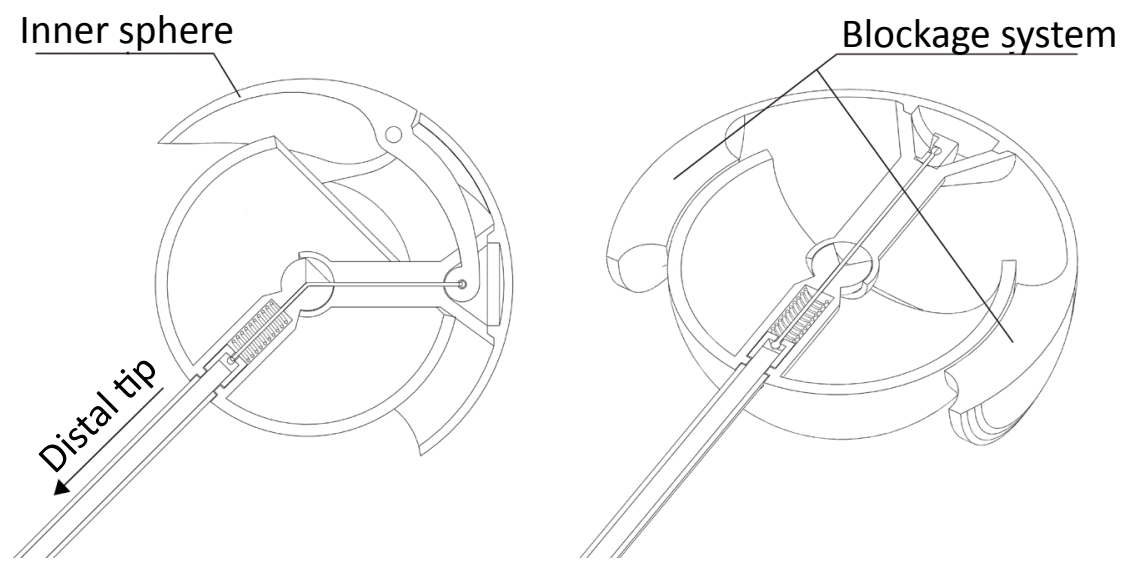

Figure 81. Sagittal and transversal planes sections, $0^{\circ}$ and $45^{\circ}$ view. PF prototype (Evotool). 
The instrument configuration makes possible to move the distal tip inside the abdomen without great changes in the upper limb position, maintaining the wrist straight and the arm and forearm inside the rang of neutral positions. When these movements are compared with the conventional instruments the differences becomes evident (Figure 82). The conventional instrument forces the surgeons to maintain the arm abducted and the wrist flexed in the majority of the positions evaluated.

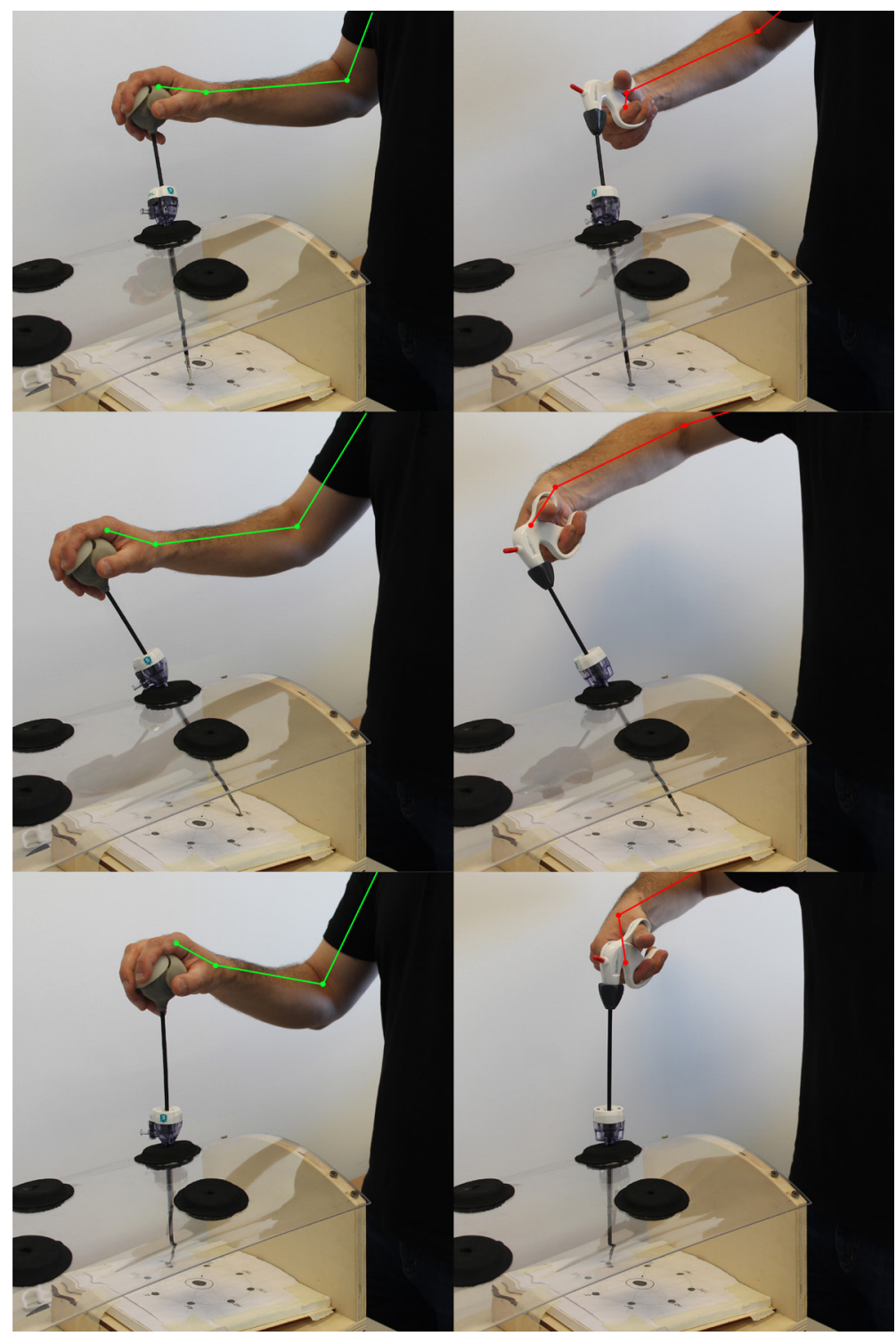

Figure 82. Comparison for two positions with the Evotool prototype and a conventional instrument. In the figure a) the conventional instrument reach the target 270 and figure b) the PF prototype reaching the same target. In figure c) a different perspective with the conventional instrument reaching the target 90 and figure d) the PF prototype reaching the same target. 
The instrument tip can be rotate using the blockage system and slightly turning the wrist to reach the right orientation. Once reached, the surgeon can recover the neutral position and continue working (Figure 83).

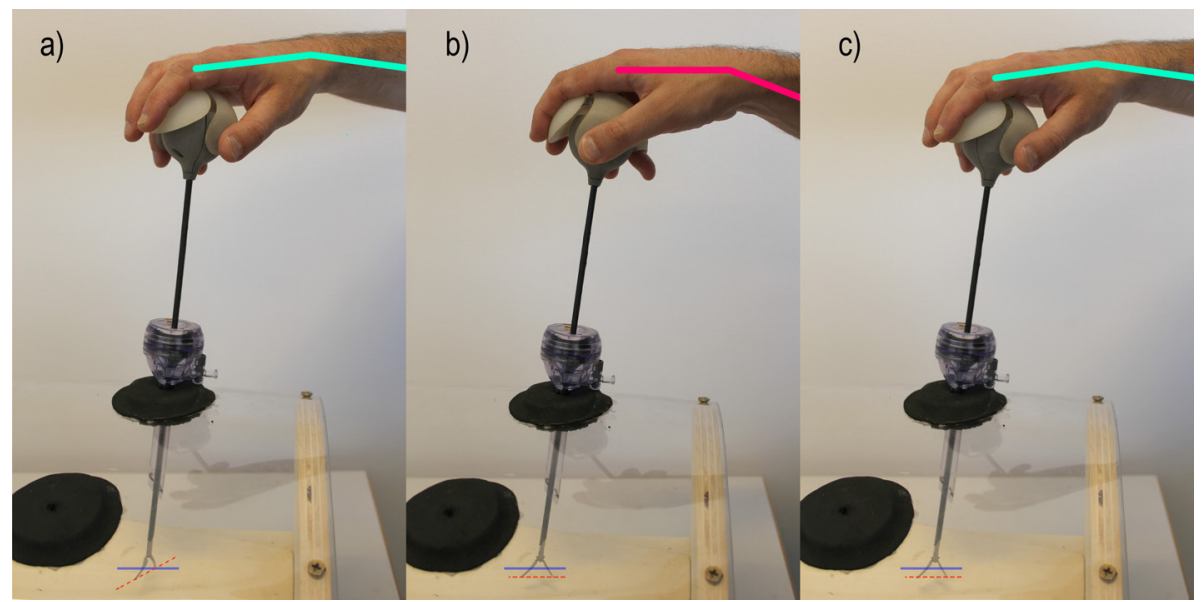

Figure 83. Evotool rotation steps. With Evotool, a) if the surgeon needs to change the angle of the distal tip, is juts necessary $b$ ) to slightly turn the wrist in order to reach the appropriate angle, once is reached c) the surgeon can recover a position more comfortable for the wrist.

As explained before, an optimal instrument has to reach some goals [61]:

- Enables the surgeon to keep both wrists in a neutral position.

- Permits the surgeon to keep both arms at the sides of their body.

- Avoids pressure points on the hands.

- Allows the surgeon to apply force with a power grip hand position.

- Allows fine manipulation with a precision grip hand position.

The first 3 points are already accomplished by the new instrument but there is other two that has to be reviewed with experts surgeons during some simulation tasks to confirm the usability of the new instrument 


\section{Evaluating the solution with real users}

\subsection{User-based evaluations}

With the final design established is time to perform a user-based evaluation in order to polish the different characteristics of the design.

User-based evaluations are usability evaluation methods in which users directly participate. These users are invited to do typical tasks with a product, or simple asked to explore it freely, while their behaviors are observed and recorded in order to identify design flaws that cause user errors or difficulties [183].

The implementation of a user test generally goes through a certain number of steps such as [183]:

- The definition of the test objectives

- The qualification and recruitment of tests participants

- The selection of tasks participants will have to realize

- The creation and description of the task scenarios

- The choice of the measures that will be made as well as the way data will be recorded

- The preparation of the test materials and of the test environment (the usability laboratory)

- The choice of the tester, and the design of the test protocol per se (instructions, design protocol, etc.) 
- The design and/or the selection of satisfaction questionnaires, the data analyses procedures

- The presentation and communication of the test results

This approach recognize the degree of satisfaction and the possible changes that the final users need on the prototype. It ensures that the prototype is adapted to the users, their tasks and that there are no negative outcomes of their usage [183].

\subsection{Test with expert users}

In order to receive feedback of surgeons and residents of surgery about the Evotool prototype, a user test was developed.

The goal of this test was to know their perception about the Evotool prototype on its basic state (Figure 84), in the second part of the experiment, the participants indicate the most suitable shape of the instrument handle. In this study, all the participants has previous knowledge in laparoscopic surgery or simulation.

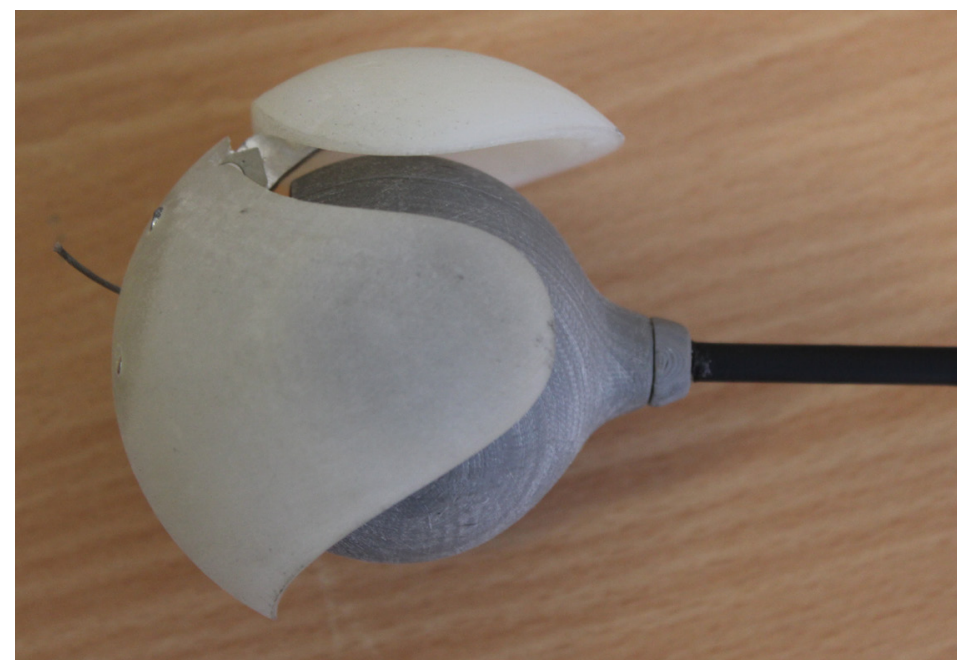

Figure 84. Prototype used with the final users. It was a functional prototype in an early stage. The users can interact with it and use it to perform simple tasks. 
During the experiment, the participants performed a pick and place test (Figure 85) with different objects in a box-trainer during some minutes. Two bowls were located inside the box-trainer, one of those bowls was full of pins and the other was empty, they had to pick the pins one by one and leave it in the empty bowl.

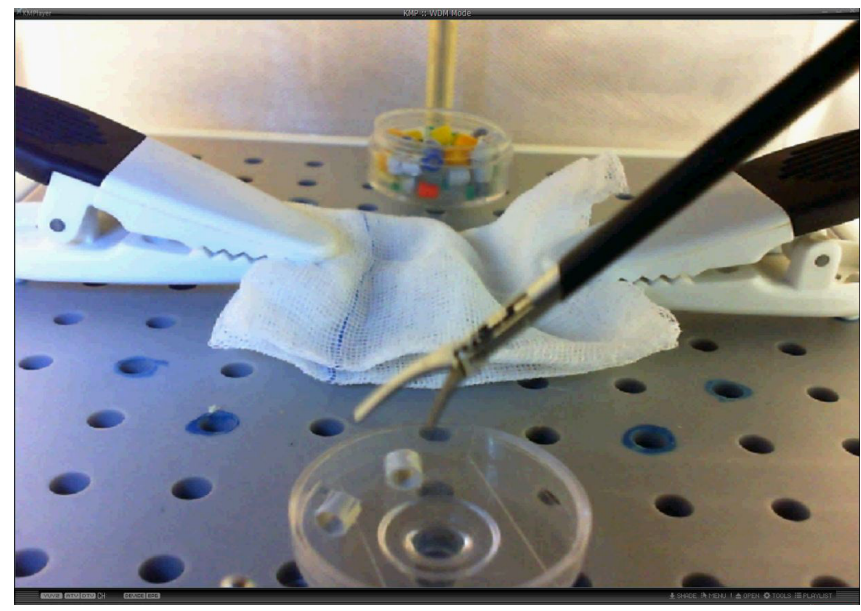

Figure 85. Pick and place test performed in the Hospital la Fe. The video was obtained from the laparoscope record.

The participants believed that was important the amount of objects correctly placed in the second bowl but it was just to make them perform movements as accurate as possible with the Evotool prototype. They performed the pick and place test during 5 minutes (Figure 86).

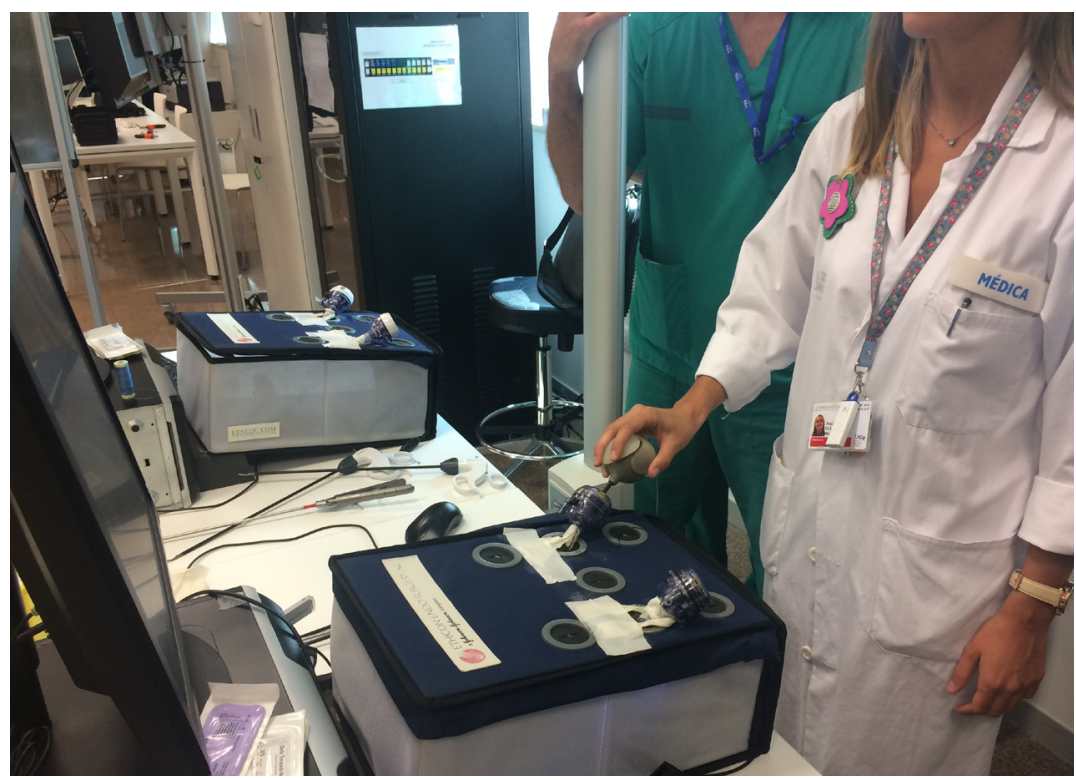

Figure 86. Surgeons performing the pick and place test before the survey to test the usability of the PF prototype. 
The day of the experiment, the participant received an explanation of the pick and place test and they had time to see the instruments, the location of the bowls and the pins. Then the trocar was located according to the dominant arm of the participant and they performed the test during 5 minutes trying to move as much pins as they could. Once finished, the participant was moved to a different location to complete the survey and a different participant performed the pick and place test. In the survey, they had to indicate their feelings with the Evotool prototype and two different handle configurations (Figure 87). The different parts of the prototypes were manufactured by means of two different 3D printing machines: Zortrax M200 (Zortrax, Poland) and Stratasys Object30 (Stratasys, USA). A final manual post-process was performed in order to improve the quality of the surface.

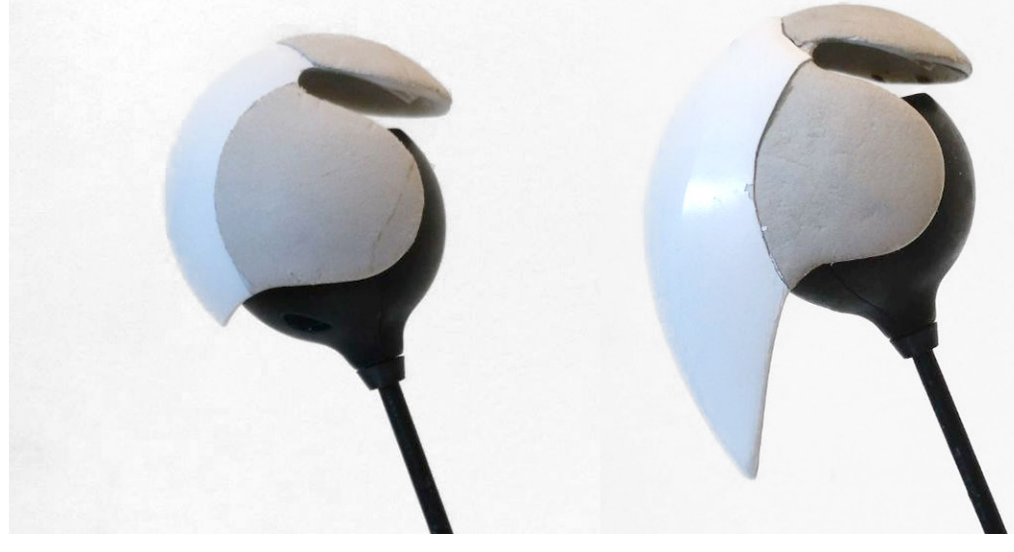

Figure 87. The two handle design alternatives used during the second part of the experiment. Model 1 left side and Model 2 right side.

The questions included in the survey were:

- What is your gender?

- What is your dominant hand?

- How much time are you doing laparoscopic surgery?

- Do you think is comfortable the system to open and close the grasper of the Evotool prototype in comparison with the conventional instruments?

- Do you think is comfortable the rotating system of the PF prototype in comparison with the conventional instruments? 
- Overall, do you think is comfortable the handle shape?

- What do you think about the size of the handle?

- How accurate do you think you could be with the Evotool prototype in comparison with the conventional instruments?

- If you select less accurate, do you think would be possible to reach similar accuracy with more training time?

- How do you think is the idea to implement an articulation in the handle to improve your posture during the surgery?

- Which one of the two Evotool handles do you prefer?

- Which actuators configuration do you prefer?

- If we can solve the problems you found in the Evotool prototypes and you have the opportunity to choose one of those, which one would you prefer to use during a surgery?

- What is your overall valuation of the Evotool prototype?

- Do you have other suggestion that would be useful to us in order to improve the instrument?

In order to quantify the results of the survey, it was coded and the results introduced in the SPSS software in order to perform a statistical analysis. The survey includes multiple choice question, liker-type scales and open-ended questions.

\subsection{Test Results}

The survey answers are presented in Table 7 were results of surgeons and residents on the Hospital la Fe are presented, $58,3 \%$ of the surveyed were females and $41,7 \%$ males, all the surveyed were right handed. According to the results obtained, 58,3\% of the participants had less than 1 year of experience, $25 \%$ had experience between 1 and 3 years and $16,7 \%$ more than 3 years of experience. 
The results of the survey showed that the $100 \%$ (33,3\% comfortable and $66,7 \%$ very comfortable) of the participant think that the trigger used in the instrument to open and close the distal tip is comfortable, in contrast to the rotation system, which only $50 \%$ think it is comfortable ( $25 \%$ comfortable and $25 \%$ very comfortable). $58,3 \%$ of the surveyed think that the size of the handle is appropriate. They also show their disagreement with the accuracy of the instrument $(41,7 \%$ think that the instrument is less accuracy and just $16,7 \%$ think is more accuracy). $100 \%$ of the surveyed think that is good idea to include an articulation to improve their posture during the surgery.

About the different handle's shape, the $91,6 \%$ of the surveyed preferred one of the new handle shapes, being the model 2 most popular. But the current actuators configuration seen not to convince the greatest part of the surveyed.

Table 7. Survey answers by the surgeons and residents of the Hospital la Fe.

\begin{tabular}{|c|c|c|c|}
\hline & & № & $\%$ \\
\hline \multirow{2}{*}{$\begin{array}{l}\text { QUESTION1. } \\
\text { GENDER }\end{array}$} & Female & 7 & $58,3 \%$ \\
\hline & Male & 5 & $41,7 \%$ \\
\hline \multirow{2}{*}{$\begin{array}{l}\text { QUESTION2. } \\
\text { DOMINANTSIDE }\end{array}$} & Left handed & 0 & $0,0 \%$ \\
\hline & Right handed & 12 & $100,0 \%$ \\
\hline \multirow{3}{*}{$\begin{array}{l}\text { QUESTION3. } \\
\text { EXPERIENCE }\end{array}$} & Less than 1 year & 7 & $58,3 \%$ \\
\hline & $1-3$ years & 3 & $25,0 \%$ \\
\hline & +3 years & 2 & $16,7 \%$ \\
\hline \multirow{5}{*}{$\begin{array}{l}\text { QUESTION4. } \\
\text { CONFORT OPEN } \\
\text { AND CLOSE }\end{array}$} & Very uncomfortable & 0 & $0,0 \%$ \\
\hline & Uncomfortable & 0 & $0,0 \%$ \\
\hline & Neutral & 0 & $0,0 \%$ \\
\hline & Comfortable & 4 & $33,3 \%$ \\
\hline & Very comfortable & 8 & $66,7 \%$ \\
\hline \multirow{5}{*}{$\begin{array}{l}\text { QUESTION5. } \\
\text { CONFORT } \\
\text { ROTATION }\end{array}$} & Very uncomfortable & 0 & $0,0 \%$ \\
\hline & Uncomfortable & 0 & $0,0 \%$ \\
\hline & Neutral & 6 & $50,0 \%$ \\
\hline & Comfortable & 3 & $25,0 \%$ \\
\hline & Very comfortable & 3 & $25,0 \%$ \\
\hline \multirow{5}{*}{$\begin{array}{l}\text { QUESTION6. } \\
\text { CONFORT } \\
\text { GENERAL }\end{array}$} & Very uncomfortable & 0 & $0,0 \%$ \\
\hline & Uncomfortable & 0 & $0,0 \%$ \\
\hline & Neutral & 0 & $0,0 \%$ \\
\hline & Comfortable & 5 & $41,7 \%$ \\
\hline & Very comfortable & 7 & $58,3 \%$ \\
\hline
\end{tabular}




\begin{tabular}{|c|c|c|c|}
\hline \multirow[t]{5}{*}{ QUESTION7. SIZE } & Too low & 0 & $0,0 \%$ \\
\hline & Low & 2 & $16,7 \%$ \\
\hline & Appropriate & 7 & $58,3 \%$ \\
\hline & Big & 3 & $25,0 \%$ \\
\hline & Too big & 0 & $0,0 \%$ \\
\hline \multirow{3}{*}{$\begin{array}{l}\text { QUESTION8. } \\
\text { ACCURACY }\end{array}$} & Less accurate & 5 & $41,7 \%$ \\
\hline & Same accurate & 5 & $41,7 \%$ \\
\hline & More accurate & 2 & $16,7 \%$ \\
\hline \multirow{5}{*}{$\begin{array}{l}\text { QUESTION10. } \\
\text { IDEA TO IMPROVE } \\
\text { POSTURE }\end{array}$} & Very bad & 0 & $0,0 \%$ \\
\hline & Bad & 0 & $0,0 \%$ \\
\hline & Irrelevant & 0 & $0,0 \%$ \\
\hline & Good & 6 & $50,0 \%$ \\
\hline & Very good & 6 & $50,0 \%$ \\
\hline \multirow{2}{*}{$\begin{array}{l}\text { QUESTION11. } \\
\text { MODEL PREF. }\end{array}$} & Model 1 & 4 & $33,3 \%$ \\
\hline & Model 2 & 8 & $66,7 \%$ \\
\hline \multirow{3}{*}{$\begin{array}{l}\text { QUESTION12. } \\
\text { PUSH BUTTONS } \\
\text { PREFERENCES }\end{array}$} & $\begin{array}{c}\text { Trigger at the top and } \\
\text { blockage at laterals }\end{array}$ & 5 & $41,7 \%$ \\
\hline & $\begin{array}{c}\text { Trigger at laterals and } \\
\text { blockage at the top }\end{array}$ & 5 & $41,7 \%$ \\
\hline & $\begin{array}{c}\text { Different combination } \\
\text { with similar or } \\
\text { different buttons }\end{array}$ & 2 & $16,7 \%$ \\
\hline \multirow{3}{*}{$\begin{array}{l}\text { QUESTION13. } \\
\text { BEST HANDLE }\end{array}$} & Conventional & 1 & $8,3 \%$ \\
\hline & Model 1 & 4 & $33,3 \%$ \\
\hline & Model 2 & 7 & $58,3 \%$ \\
\hline \multirow{3}{*}{$\begin{array}{l}\text { QUESTION14. } \\
\text { PROTOTIPE } \\
\text { ASSESSMENT }\end{array}$} & 3,00 & 4 & $33,3 \%$ \\
\hline & 4,00 & 5 & $41,7 \%$ \\
\hline & 5,00 & 3 & $25,0 \%$ \\
\hline
\end{tabular}

\subsection{Discussion}

The goal of the user test was to evaluate a new concept design for laparoscopic surgery. The appropriate participants for this study were experienced surgeons and residents with knowledge of the laparoscopic procedures. 
The scarcity of participants made this study not representative of the occupational group. Nevertheless, the data generated by the experiment show interesting results to discussed about some of the premises that the research team used to create the Evotool design.

According to the results the new instrument is a good solution for the participants, because $100 \%$ of those were agree that the design was comfortable and that is a good idea to implement the PF characteristic to laparoscopic instruments.

In spite of the good embrace of the PF patented design, just $58,3 \%$ of the participants though that the size was appropriate which means that different sizes should be provided to adapt it to different hand sizes. Also, just $50 \%$ of the participants though that the rotation system was great and, taking account of the poor ergonomically rotation system of the conventional pistol-grip handles, the rotation system should be polished in order to be an important benefit of the new instrument. The accuracy of the instrument is also an important characteristic to improve, $41,7 \%$ of the participants thought that they were less accurate with the new instrument, which could be due to the lack of training, but they complained about the lack of security while they were grabbing the instrument, because they though it will falling out easily.

Different ideas can be obtained from this experiment, the first one is that the proposed PF design solution is in the right direction with the concept of PF. Surgeons think that is important to take care of their musculature and the PF concept should be implemented in future laparoscopic instruments. Second, the rotational system should be reconfigured, during the laparoscopic surgery the surgeon has to perform a variety of turns and recoveries to reach different organs, to cut and to suture, and an optimal rotational system should be easy to use and as accurate as possible. Third, some characteristics of the instrument should be customized for different users. The size of the instrument or the length of the posterior surface is not a value that could be just generically implemented, the differences between surgeons are important enough to include some variable elements in the instrument, regardless of the price or manufacture time that it supposed. 



\section{Chapter 6. Conclusions}


Laparoscopic surgery is the reality of the current surgery practices. From the conventional surgeries to the new robotic procedures all are performed following similar principles and theories to maintain the patients safe, being the patient always the focus of all the efforts to made their experience as positive as possible. This work tries to take surgeons into consideration, being they the effector of the end procedure and the responsible of the patient's life.

This thesis has presented a general review of the laparoscopic practice and the different musculoskeletal and positional problems that underlie the procedure. Two chapters focus on SEMG and MoCap analysis in order to evaluate and compare the effect on the human body of a new concept based in the benefits of increase the surgeon's freedom in the operation room. After the body evaluation, a new instrument design based has been considered on the postural freedom concept that has the potential to reduce the musculoskeletal problems inherent to laparoscopic surgery. Finally, this new concept was reviewed by some surgeons in order to know their impressions and perceptions with the final solution.

In Chapter 2 were explained the specific objectives tackled during this research, in the following lines those objectives will be answered:

- Objective G1.1. Create a database of electromyography registers in muscles trapezius, deltoids, biceps and flexor carpi radials. The registers will be obtained while the participants use two different instruments, a conventional instrument and a prototype based in the "Postural Freedom" concept, during static and dynamic experiments.

Indeed, a database of muscles trapezius, deltoids, biceps, and flexor carpi radials was created including data from each participant with both instruments in static and dynamic experiments. This database still can be enlarged including more participants and muscles but, in order to see the effect of the "Postural Freedom", twelve hours of register is enough. This is a personal contribution because the whole acquisition system and the database had been created specifically for this experiment.

- Objective G1.2. Calculate parameters to quantify the muscular activity in each muscle evaluated during the experiment. The signal will be processed in order to compare the results obtained with both instruments and to determine which one requires less effort. 
After a thoroughly search of the appropriate parameter, the root mean square was the parameter used in order to quantify the muscular activity in muscles trapezius, deltoids, biceps, and flexor carpi radials. The signal was processed to compare both instruments. The results indicated that the "Postural Freedom" prototype reduced the muscular activity in all the muscles evaluated, being more evident in trapezius and deltoids muscles, key muscles for the movements performed in laparoscopic surgery. This is a personal contribution, the protocol and the prototype were created for this experiment.

- Objective G1.3. Calculate parameters in the spectral domain to quantify the muscular fatigue in each muscle evaluated during the experiment. The signal will be processed in order to compare the results obtained with both instruments and to determine which one produce less muscular fatigue.

Several parameters were tested in order to calculate the muscular fatigue. Finally the median frequency parameter was used, because it was more appropriate for the purpose of the experiment. The signal was processed to compare both instruments. The results indicate that the "Postural Freedom" prototype has the potential to reduce the muscular fatigue generated during laparoscopic surgery. This improvement was evident after just 10 minutes of experiment, which means that this reduction in long-term laparoscopic process would be largest. This is a personal and original contribution because the protocol and the prototype evaluated were created for this experiment.

- Objective G2.1. Define the setup for a motion capture experiment that can be used during dynamic laparoscopic simulations, without interfere in the participants movements.

Indeed, after several experiments a setup for a dynamic motion capture experiment was defined. This setup do not interfere with the participants movements and can be easily replicated. The robustness of the setup employed during this experiment would allow it to, after a few modifications of the kind of markers used, be used to evaluate surgeons inside the operating room. This is a personal contribution because the acquisition setup and the prototype were created for this experiment. 
- Objective G2.2. Register and evaluate the trajectories followed by each section of the dominant limb during the simulations with a conventional instrument and a prototype based on the "Postural Freedom" concept.

The trajectories performed by dominant arms of each participant of the motion capture experiment were registered and evaluated. The conventional instrument and the "Postural Freedom" prototype were used during the experiment. Using the existing literature we identified the critical positions and the system can indicate us which movements were potentially dangerous for their musculoskeletal system. The setup using during this experiment and the prototype were personal and original contributions, the surgeons' team of the Hospital La Fe were vital to identify the movements to register.

- Objective G2.3. Estimate the effect of a laparoscopic instrument based in the "Postural Freedom" concept in the participants" postural health in comparison with the conventional laparoscopic instruments.

Certainly, the effect of a "Postural Freedom" prototype was measured in order to estimate its effect on participants' postural health. The results indicated that the "Postural Freedom" prototype reduced the critical displacement presented in conventional instruments, and kept the participants' extremities in neutral positions during greater part of the experiment. This is a personal and original contribution because both, the setup used and the protocol were prepared specifically for this experiment.

- Objective G3.1. Design of a new instrument based on "Postural Freedom" concept and develop a fully functional prototype based in the knowledge acquired with the experimentation and the feedback provided by expert users.

A new concept of instrument based in the "Postural Freedom" concept was designed and patented. This new design was called "Evotool", it is a spherical instrument with a variable handle-to-shaft angle. At this stage, the intention was to manufacture a fully functional prototype of "Evotool" to be tested by users. Due to a lack of mechanical refinement caused by friction between parts, only a partial prototype of the trigger actuator and the ball-joint was prototyped. The design proposed is a personal and original contribution based in our experimentation. 
- Objective G3.2. Evaluate the fully functional prototype with experts and novices surgeons in order to test their satisfaction with the final solution.

A pick and place test was carried out and the level of satisfaction experimented was evaluated. Even though the users could not perform accurately turns of the instrument's distal tip due to frictional problems, the results of the evaluations showed positive results in regards to the satisfaction with the new concept. The feedback obtained will be essential for the development of the fully functional prototype that will include variations based on the feedback obtained during the experiment. This is a personal contribution because the instrument and the interviews were prepared specifically for this experiment.

These specific objectives were defined in accordance to the three general objectives determined for this research:

- The electromyography study showed that an instrument based in the "Postural Freedom" concept reduces the muscular activity and the localized muscular fatigue in arm and back muscles during a laparoscopic static exercise.

- The motion capture study indicated that an instrument based in the "Postural Freedom" concept reduces the amount of critical displacement and maintain the upper limb's motions in neutral position. Reducing the risk of musculoskeletal disorders caused by the poor ergonomic postures maintained in laparoscopic surgery.

- Base on first conclusions, a new instrument design called "Evotool" was patented and prototyped. This new design was evaluated by expert surgeons and the results were positive

The results presented in this manuscript suggest that the postural freedom concept could be a useful characteristic to increase the neutral postures and reduce the effort and muscular fatigue during the laparoscopic procedures but it should be refined to reach and surpass the accuracy presented with the conventional instruments. 
The solution presented by the research team is not the only possible solution, the medical device companies working in the laparoscopic hand-held instrumentation field should take in consideration the results presented on this manuscript to implement the postural freedom concept on their devices.

Based in the work performed during this research, future lines of work have been suggested:

- Leveraging the SEMG registers, the parameters used to measure muscle fatigue should be redefined. New muscular fatigue indicators that are able to discreet between different kinds of fatigue and enlighten the localized muscular fatigue should be established.

- The dynamic protocol and the signal process should be improved in order to obtain reliable data and conclusions from real procedures.

- Using the non-invasive motion capture system, a study evaluating simultaneously both electromyography signal and limb displacements during a laparoscopic simulation should be performed.

- Using different systems, as inertial sensors for motion capture evaluation or infrared thermography for muscular fatigue evaluations, compare the results obtained with results both in simulation and real procedures.

- Using pressure sensors, a study evaluating the effort required to handle the new instrument design should be performed.

- The mechanism should be optimized for advanced prototypes. This will require the collaboration with a mechanical engineer department.

- The handle shape should be optimized for the surgeons' handles. For this purpose, the location and geometry of the different actuators and the external structure should be tested in real environment. 




\section{Chapter 7. \\ Bibliography}


[1] H. Marcovitch, Ed., Black's Medical Dictionary, 41st Editi. London: A \& C Black, 2005.

[2] A. Najmaldin and P. Guillou, A Guide to Laparoscopic Surgery, vol. 82, no. 5. 1998.

[3] A. DiMartino, K. N. Done', T. . Judkins, J. Morse, and J. Melander, "Ergonomic Laparoscopic Tool Handle Design," Hum. Factors, vol. 48, no. 12, pp. 1354-1358, 2004.

[4] S. Papadoukakis, D. Kusche, and M. C. Truss, "History of Laparoscopy, Endoscopic Extraperitoneal Radical Prostatectomy and Robotic Surgery," Endosc. Extraperitoneal Radic. Prostatectomy, pp. 1-9, 2007.

[5] H. Gao and Z. Zhang, "Laparoscopy Versus Laparotomy in the Treatment of High-Risk Endometrial Cancer: A Propensity Score Matching Analysis," Med., vol. 94, no. 30, p. e1245, 2015.

[6] R. Berguer, D. Forkey, and W. Smith, "Ergonomics Problems associated with Laparoscopy Surgery," Surg Endosc, vol. 13, no. 5, pp. 466-8, 1999.

[7] R. Berguer, "Surgery and ergonomics," Arch. Surg., vol. 134, no. 9, pp. 1011-1016, 1999.

[8] M. S. Raghu Prasad, M. Manivannan, and S. M. Chandramohan, "Effects of laparoscopic instrument and finger on force perception: a first step towards laparoscopic force-skills training," Surg. Endosc. Other Interv. Tech., pp. 1927-1943, 2014.

[9] H. C. Jacobaeus, "Über die Möglichkeit die Zystoskopie bei Untersuchung seröser Höhlungen anzuwenden," Munch Med Wochenschr, vol. 57, pp. 2090-2092, 1910.

[10] H. C. Jacobaeus, "Kurze Uebersicht über meine Erfahrungen mit der Laparo-thoraskopie," Minch Med Wochenschr, vol. 58, pp. 2017-2019, 1911.

[11] A. Toro, M. Mannino, G. Cappello, A. Di Stefano, and I. Di Carlo, "Comparison of two entry methods for laparoscopic port entry: Technical point of view," Diagn. Ther. Endosc., vol. 2012, 2012.

[12] W. U. Wayand, "Jànos Veres: The man behind the needle," Surg. Endosc. Other Interv. Tech., vol. 28, no. 2, pp. 351-352, 2014. 
[13] H. J. Bonjer, E. J. Hazebroek, G. Kazemier, M. C. Giuffrida, W. S. Meijer, and J. F. Lange, "Open versus closed establishment of pneumoperitoneum in laparoscopic surgery," Br. J. Surg., vol. 84, no. 5, pp. 599-602, 1997.

[14] S. D. St. Peter and G. W. Holcomb, History of Minimally Invasive Surgery, no. January 2009. Elsevier Inc., 2008.

[15] D. R. K. Mishra, "World Laparoscopy Hospital." [Online]. Available: https://www.laparoscopyhospital.com.

[16] N. Katkhouda et al., "Laparoscopic versus open appendectomy: A prospective randomized double-blind study," Ann. Surg., vol. 242, no. 3, pp. 439-450, 2005.

[17] J. L. Flowers et al., "Comparison of open and laparoscopic live donor nephrectomy.," Ann. Surg., vol. 226, no. 4, pp. 483-9; discussion 489-90, 1997.

[18] R. Berguer, G. T. Rab, A. Alarcon, and J. Chung, "A comparison of surgeons ' posture during laparoscopic and open surgical procedures," Sport. Med., vol. 11, pp. 139-142, 1997.

[19] N. T. Nguyen et al., "An ergonomic evaluation of surgeons' axial skeletal and upper extremity movements $\backslash$ par during laparoscopic and open surgery. \par," Am J Surg, vol. 182\par, no. 6\par, p. 720-4 \par, 2001.

[20] R. Berguer, D. L. Forkey, and W. D. Smith, "Ergonomic problems associated with laparoscopic surgery.," Surg. Endosc., vol. 13, no. 5, pp. 466-468, May 1999.

[21] L. P. Aitchison, J. Flint, E. Nesbitt-Hawes, W. Ledger, and J. Abbott, "a Feasibility Study Determining Surgical Ergonomics in a Live Surgical Setting," J. Minim. Invasive Gynecol., vol. 22, no. 4, pp. 626-630, 2015.

[22] C. J. De Luca, "Myoelectrical manifestations of localized muscular fatigue in humans.," Crit. Rev. Biomed. Eng., vol. 11, no. 4, pp. 251-279, 1984.

[23] B. Muscio, "IS A FATIGUE TEST POSSIBLE?," Br. J. Psychol. Gen. Sect., vol. 12, no. 1, pp. 31-46, 1921.

[24] A. G. Bills, "The psychology of efficiency; a discussion of the hygiene of mental work.," 1943. 
[25] D. B. Chaffin, "Localized muscle fatigue-definition and measurement," Journal of Occupational and Environmental Medicine, vol. 15. pp. 346-354, 1973.

[26] B. Bigland-Ritchie and J. J. Woods, "Changes in muscle contractile properties and neural control during human muscular fatigue.," Muscle \& nerve, vol. 7, no. 9. pp. 691-9, 1984.

[27] B. Bigland Ritchie, D. A. Jones, G. P. Hosking, and R. H. T. Edwards, "Central and peripheral fatigue in sustained maximum voluntary contractions of human quadriceps muscle," Clin. Sci. Mol. Med., vol. 54, no. 6, pp. 609-614, 1978.

[28] R. M. Enoka and J. Duchateau, "Muscle fatigue: what, why and how it influences muscle function," J Physiol, vol. 5861, pp. 11-23, 2008.

[29] S. Rodbard and E. B. Pragay, "Contraction frequency, blood supply, and muscle pain," J. Appl. Physiol., vol. 24, no. 2, pp. 142-5, Feb. 1968.

[30] S. P. Cairns, A. J. Knicker, M. W. Thompson, and G. Sjogaard, "Evaluation of models used to study neuromuscular fatigue," Exerc. Sport Sci. Rev., vol. 33, no. 1, pp. 9-16, 2005.

[31] R. M. Enoka and D. G. Stuart, "Neurobiology of muscle fatigue," J. Appl. Physiol., vol. 72, no. 5, pp. 1631-1648, 1992.

[32] T. A. Emam, T. G. Frank, G. B. Hanna, and A. Cuschieri, "Influence of handle design on the surgeon's upper limb movements, muscle recruitment, and fatigue during endoscopic suturing.," Surg. Endosc., vol. 15, no. 7, pp. 667-672, 2001.

[33] A. Cuschieri, "Whither minimal access surgery: tribulations and expectations," Am. J. Surg., vol. 169, no. 1, pp. 9-19, 1995.

[34] M. L. Uhrich, R. A. Underwood, J. W. Standeven, N. J. Soper, and J. R. Engsberg, "Assesment of fatigue, monitor placement, and surgical experience during simulated laparoscopic surgery," Surg. Endosc. Other Interv. Tech., vol. 16, no. 4, pp. 635-639, 2002.

[35] R. Berguer, M. Remler, and D. Beckley, "Laparoscopic instruments cause increased forearm fatigue: A subjective and objective comparison of open and laparoscopic techniques," Minim. Invasive Ther. Allied Technol., vol. 6, no. 1, pp. 36-40, 1997. 
[36] R. Berguer, "Surgical technology and the ergonomics of laparoscopic instruments," Surg. Endosc., no. 12, pp. 458-462, 1998.

[37] S. Ahmed, G. B. Hanna, and A. Cuschieri, "Optimal angle between instrument shaft and handle for laparoscopic bowel suturing.," Arch. Surg., vol. 139, no. 1, pp. 89-92, 2004.

[38] G. B. Hanna, S. Shimi, and A. Cuschieri, "Optimal port locations for endoscopic intracorporeal knotting.," Surg. Endosc., vol. 11, no. 4, pp. 397-401, Apr. 1997.

[39] M. W. Stomberg et al., "Work-related musculoskeletal disorders when performing laparoscopic surgery.," Surg. Laparosc. Endosc. Percutan. Tech., vol. 20, no. 1, pp. 49-53, 2010.

[40] G. Lee, T. Lee, D. Dexter, R. Klein, and A. Park, "Methodological infrastructure in surgical ergonomics: a review of tasks, models, and measurement systems.," Surg. Innov., vol. 14, no. 3, pp. 153-167, 2007.

[41] D. Koca et al., "Physical and Mental Workload in Single-Incision Laparoscopic Surgery and Conventional Laparoscopy," Surg. Innov., vol. 22, no. 3, pp. 294-302, 2015.

[42] N. Stanton, A. Hedge, K. Brookhuis, E. Salas, and H. Hendrick, Handbook of Human Factors and Ergonomics Methods. 2005.

[43] A. Phinyomark, S. Thongpanja, H. Hu, P. Phukpattaranont, and C. Limsakul, "The Usefulness of Mean and Median Frequencies in Electromyography Analysis," in Computational Intelligence in Electromyography Analysis-A Perspective on Current Applications and Future Challenges, 2012, pp. 195-220.

[44] T. J. Armstrong et al., "A conceptual model for work-related neck and upper-limb musculoskeletal disorders," Scand. J. Work Environ. Heal., vol. 19, no. 2, pp. 73-84, 1993.

[45] B. Visser and J. H. Van Dieën, "Pathophysiology of upper extremity muscle disorders," J. Electromyogr. Kinesiol., vol. 16, no. 1, pp. 1-16, 2006.

[46] V. Putz-Anderson et al., "A Critical Review of Epidemiologic Evidence for Work-Related Musculoskeletal Disorders of the Neck, Upper Extremity, and Low Back," 1997. 
[47] A. van der Beek and M. Frings-Dresen, "Assessment of mechanical exposure in ergonomic epidemiology.," Occup. Environ. Med., vol. 55, no. 5, pp. 291-299, 1998.

[48] G. C. David, "Ergonomic methods for assessing exposure to risk factors for work-related musculoskeletal disorders," Occup. Med. (Chic. III)., vol. 55, no. 3, pp. 190-199, 2005.

[49] R. Wells, R. Norman, P. Neumann, and D. Andrews, "Assessment of physical work load in epidemiologic studies: common measurement metrics for exposure assessment," Ergonomics, vol. 37, no. 6, pp. 979-88, 1997.

[50] R. Merletti, M. Knaflitz, and C. J. De Luca, “Myoelectric manifestations of muscle fatigue in voluntary and electrically elicited contractions," J. Appl. Physiol., vol. 69, no. 5, pp. 1810-20, 1990.

[51] G. Li and P. Buckle, "Current techniques for assessing physical exposure to work-related musculoskeletal risks, with emphasis on posture-based methods," Ergonomics, vol. 42, no. 5, pp. 674-695, 1999.

[52] A. G. Kirk, J. F. O’Brien, and D. A. Forsyth, "Skeletal Parameter Estimation from Optical Motion Capture Data," 2005 IEEE Comput. Soc. Conf. Comput. Vis. Pattern Recognit., vol. 2, pp. 782-788, 2005.

[53] World Health Organization, "Patient safety curriculum guide. Multi-Professional Edition.," Paitent Saf., pp. 1-272, 2011.

[54] M. Patkin and L. Isabel, "Ergonomics, engineering and surgery of endosurgical dissection," J. R. Coll. Surg. Edinb., vol. 40, pp. 120132, 1995.

[55] M. Patkin, "Ergonomic Aspects of Surgical Dexterity," Med. J. Aust., vol. 2, no. 775, 1967.

[56] G. P. Y. Szeto, S. W. K. Cheng, J. T. C. Poon, A. C. W. Ting, R. C. C. Tsang, and P. Ho, "Surgeons' static posture and movement repetitions in open and laparoscopic surgery," J. Surg. Res., vol. 172, no. 1, pp. e19-e31, 2012.

[57] F. Tendick, R. W. Jennings, G. Tharp, and L. Stark, "Sensing and Manipulation Problems in Endoscopic Surgery: Experiment, Analysis, and Observation," Presence, vol. 2. pp. 66-81, 1993. 
[58] L. S. G. L. Wauben, M. A. Van Veelen, D. Gossot, and R. H. M. Goossens, "Application of ergonomic guidelines during minimally invasive surgery: A questionnaire survey of 284 surgeons," Surg. Endosc. Other Interv. Tech., vol. 20, no. 8, pp. 1268-1274, 2006.

[59] A. Park, G. Lee, F. J. Seagull, N. Meenaghan, and D. Dexter, "Patients Benefit While Surgeons Suffer: An Impending Epidemic," J. Am. Coll. Surg., vol. 210, no. 3, pp. 306-313, 2010.

[60] K. Miller, M. Benden, A. Pickens, E. Shipp, and Q. Zheng, "Ergonomics Principles Associated With Laparoscopic Surgeon Injury/IIIness," Hum. Factors Ergon. Soc., vol. 54, no. 6, pp. 10871092, 2012.

[61] R. Berguer, Ergonomics in Laparoscopy Surgery. New York, NY, 2006.

[62] R. Berguer, D. L. Forkey, and W. D. Smith, "The effect of laparoscopic instrument working angle on surgeons' upper extremity workload," Surg. Endosc., vol. 15, no. 9, pp. 1027-1029, 2001.

[63] V. Putz-Anderson, B. Bernard, and S. Burt, "A Critical Review of Epidemiologic Evidence for Work-Related Musculoskeletal Disorders of the Neck, Upper Extremity, and Low Back," 1997.

[64] U. Matern and P. Waller, "Instruments for minimally invasive surgery: Principles of ergonomic handles," pp. 174-182, 1998.

[65] van Veelen, D. W. Meijer, R. H. Goossens, and C. J. Snijders, "New ergonomic design criteria for handles of laparoscopic dissection forceps.," J. Laparoendosc. Adv. Surg. Tech., vol. 11, no. 1, pp. 17-26, 2001.

[66] J. Sauer and A. Sonderegger, "The influence of prototype fidelity and aesthetics of design in usability tests: Effects on user behaviour, subjective evaluation and emotion," Appl. Ergon., vol. 40, no. 4, pp. 670-677, 2009.

[67] S. Ahmed, G. B. Hanna, and A. Cuschieri, "Optimal Angle between Instrument Shaft and Handle for Laparoscopic Bowel Suturing," Arch. Surg., vol. 139, no. 1, pp. 89-92, 2004. 
[68] T. . Judkins, A. DiMartino, K. Done, M. S. Hallbeck, and D. Oleynikov, "Effect of handle design and target location on wrist posture during aiming with a pararoscopic tool," Hum. Factors Ergon. Soc. 48th Annu. Meet., pp. 1464-1468, 2004.

[69] A. Trejo, M.-C. Jung, D. Oleynikov, and M. S. Hallbeck, "Effect of handle design and target location on insertion and aim with a laparoscopic surgical tool.," Appl. Ergon., vol. 38, no. 6, pp. 745753, 2007.

[70] S. R. Herring, A. E. Trejo, and M. S. Hallbeck, "Evaluation of four cursor control devices during a target acquisition task for laparoscopic tool control," Appl. Ergon., vol. 41, pp. 47-57, 2010.

[71] I. Engineering, "Arm Posture and Muscular Activity While Inserting and Aiming with Laparoscopic Tools," vol. 3, pp. 1399-1403, 2015.

[72] S. Awtar, T. T. Trutna, J. M. Nielsen, R. Abani, and J. Geiger, "FlexDexTM: A Minimally Invasive Surgical Tool With Enhanced Dexterity and Intuitive Control," J. Med. Device., vol. 4, no. 3, p. 035003, 2010.

[73] P. L. Anderson, R. A. Lathrop, S. D. Herrell, and R. J. Webster, "Comparing a Mechanical Analogue With the Da Vinci User Interface: Suturing at Challenging Angles," IEEE Robot. Autom. Lett., vol. 1, no. 2, pp. 1060-1065, 2016.

[74] D. A. Hendrickson, "Complications of Laparoscopic Surgery," Vet. Clin. North Am. Equine Pract., vol. 24, no. 3, pp. 557-571, 2008.

[75] D. A. G. Reyes, B. Tang, and A. Cuschieri, "Minimal access surgery (MAS)-related surgeon morbidity syndromes," Surg. Endosc. Other Interv. Tech., vol. 20, no. 1, pp. 1-13, 2006.

[76] L. P. Aitchison, C. K. Cui, A. Arnold, E. Nesbitt-Hawes, and J. Abbott, "The ergonomics of laparoscopic surgery: a quantitative study of the time and motion of laparoscopic surgeons in live surgical environments," Surg. Endosc. Other Interv. Tech., vol. 30, no. 11, pp. 5068-5076, 2016.

[77] J. D. Bronzino, Biomedical Engineering Fundamentals, 3th Editio. Hartford, Connecticut, USA: CRC Press. Taylor \& Francis Group, 2006.

[78] J. Hall and C. Guyton, Textbook of medical physiology, 12th ed. Elsevier, 2010. 
[79] R. L. Lieber, Skeletal muscle structure, function, and plasticity. Lippincott Williams \& Wilkins, 2002.

[80] N. E. Quick, J. C. Gillette, R. Shapiro, G. L. Adrales, D. Gerlach, and A. E. Park, "The effect of using laparoscopic instruments on muscle activation patterns during minimally invasive surgical training procedures," Surg. Endosc. Other Interv. Tech., vol. 17, no. 3, pp. 462-465, 2003.

[81] N. Kano, T. Yamakawa, Y. Ishikawa, N. Miyajima, S. Ohtaki, and H. Kasugai, "Prevention of laparoscopic surgeon's thumb," Surg Endosc, vol. 10, pp. 1253-1255, 1996.

[82] N. Kano, T. Yamakawa, and H. Kasugai, "Laparoscopic Surgeon's Thumb," Arch. Surg., vol. 128, no. 10, p. 1172, Oct. 1993.

[83] W. J. Lee and Y. S. Chae, "Superficial nerve damage of thumb of laparoscopic surgeon.," Surg. Laparosc. Endosc. Percutan. Tech., vol. 11, no. 3, pp. 207-208, Jun. 2001.

[84] M. AW, G. JACOB, R. MR, and J. AG, "Laparoscopist's thumb: An occupational hazard," Arch. Surg., vol. 128, no. 3, p. 357, Mar. 1993.

[85] L. F. Horgan, D. C. O'riordan, and N. Doctor, "Neuropraxia following laparoscopic procedures: An occupational injury," Minim. Invasive Ther. Allied Technol., vol. 6, no. 1, pp. 33-35, 1997.

[86] D. C. van der Zee and N. M. A. Bax, "Digital nerve compression due to laparoscopic surgery," pp. 140-145, 1995.

[87] G. R. Verma, "Pressure Sore and Digital Neuropraxia of the Thumb in Laparoscopic Cholecystectomy," Surg. Laparosc. Endosc. Percutaneous Tech., vol. 14, no. 3, 2004.

[88] J. V. Basmajian and C. J. DeLuca, Muscles Alive: Their Functions Revealed by Electromyography, 5th ed. Baltimore, 1985.

[89] W. Biederman, Electrophysiology. London, 1898.

[90] A. Galvani, De Viribus Electricitatis in Motu Musculari. 1791.

[91] M. Kazamel and P. P. Warren, "History of electromyography and nerve conduction studies: A tribute to the founding fathers," J. Clin. Neurosci., vol. 43, pp. 54-60, 2017. 
[92] A. McComas, Galvani's spark: the story of the nerve impulse. Oxford University Press, 2011.

[93] G.B. Duchenne, Physiologie des mouvements. Paris, 1867.

[94] B. Bigland-Ritchie, "EMG/Force Relations and Fatigue of Human Voluntary Contractions," Exerc. Sport Sci. Rev., vol. 9, no. 1, pp. 75-118, 1981.

[95] R. Merletti and P. Parker, Electromyography : physiology, engineering, and noninvasive applications. IEEE Press, 2004.

[96] G. S. Rash, "Electromyography fundamentals," Gait Clin. Mov. Anal. Soc., 2003.

[97] C. J. De Luca, A. Adam, R. Wotiz, L. D. Gilmore, and S. H. Nawab, "Decomposition of surface EMG signals," J Neurophysiol, vol. 96, no. 3, pp. 1646-1657, 2006.

[98] J. R. Daube and D. I. Rubin, "Needle electromyography," Muscle and Nerve, vol. 39, no. 2, pp. 244-270, 2009.

[99] H. Piper, Elektrophysiologie menschlicher Muskeln. Berlin: Springer-Verlag, 1912.

[100] E. Criswell and J. R. Cram, Cram's introduction to surface electromyography. Jones and Bartlett, 2011.

[101] C. J. de Luca, "The use of surface electromyography in biomechanics," J. Appl. Biomech., vol. 13, pp. 135-163, 1997.

[102] S. Cobb and A. Forbes, "Electromyographic studies of muscular fatigue in man," Am. J. Physiol., vol. 65, no. 2, pp. 234-251, 1923.

[103] G. C. Knowlton and R. L. Bennett, "Electromyography of Fatigue.," Am. J. Phys. Med. Rehabil., vol. 31, no. 1, 1952.

[104] H. A. DeVries, "Efficiency of electrical activity as a physiological measure of the functional state of muscle tissue.," Am. J. Phys. Med., vol. 47, no. 1, pp. 10-22, Feb. 1968.

[105] R. Kadefors, E. Kaiser, and I. Petersén, "Dynamic spectrum analysis of myo-potentials and with special reference to muscle fatigue.," Electromyography, vol. 8, no. 1, pp. 39-74.

[106] O. C. J. Lippold, J. W. T. Redfearn, and J. Vuco, "The Electromyography of Fatigue," Ergonomics, vol. 3, no. 2, pp. 121-131, Apr. 1960. 
[107] J. Scherrer and A. Bourguignon, "Changes in the Electromyogram produce by Fatigue in man," Am. J. Phys. Med., vol. 38, no. 4, pp. 148-158, 1959.

[108] S. Johansson, L. E. Larsson, and R. Örtengren, "An automated method for the frequency analysis of myoelectric signals evaluated by an investigation of the spectral changes following strong sustained contractions," Med. biol. Engng, vol. 8, pp. 257-264, 1970.

[109] T. Sadoyama and H. Miyano, "Frequency analysis of surface EMG to evaluation of muscle fatigue.," Eur. J. Appl. Physiol. Occup. Physiol., vol. 47, no. 3, pp. 239-46, 1981.

[110] E. Kwatny, D. H. Thomas, and H. G. Kwatny, "An application of signal processing techniques to the study of myoelectric signals.," IEEE Trans. Biomed. Eng., vol. 17, no. 4, pp. 303-13, Oct. 1970.

[111] L. Lindstrom, R. Magnusson, and I. Petersén, "Muscular fatigue and action potential conduction velocity changes studied with frequency analysis of EMG signals.," Electromyography, vol. 10, no. 4, pp. 341-56.

[112] L. Lindström, "On the frequency spectrum of EMG signals," Chalmers Institute of Technology, Gothenburg, Sweden, 1970.

[113] C. J. de Luca, "Physiology and Mathematics of Myoelectric Signals," IEEE Trans. Biomed. Eng., vol. BME-26, no. 6, pp. 313325, 1979.

[114] R. Merletti, M. Knaflitz, and C. J. DeLuca, "Electrically evoked myoelectric signals.," Crit. Rev. Biomed. Eng., vol. 19, no. 4, pp. 293-340, 1992.

[115] J. Chang, D. Chablat, F. Bennis, and L. Ma, "Estimating the EMG response exclusively to fatigue during sustained static maximum voluntary contraction," vol. c, no. Cv, p. 27.

[116] L. H. Lindstrom and R. Magnusson, "Interpretation of Myoelectric Power Spectra : A Model and Its Applications," Proceedigns IEEE, vol. 65, no. 5, pp. 653-663, 1977.

[117] F. B. Stulen and C. J. De Luca, "Frequency Parameters of the Myoelectric Signal as a Measure of Muscle Conduction Velocity," IEEE Trans. Biomed. Eng., vol. BME-28, no. 7, pp. 515-523, 1981. 
[118] G. Balestra, M. Knaflitz, and R. Merletti, "Comparison between myoelectric signal mean and median frequency estimates," in Proceedings of the Annual International Conference of the IEEE Engineering in Medicine and Biology Society, 1988, pp. 17081709.

[119] M. Hagberg, Electromyographic signs of shoulder muscular in two elevated arm postions, vol. 60. 1981.

[120] S. R. Stock, "Workplace ergonomic factors and the development of musculoskeletal disorders of the neck and upper limbs: a meta-analysis.[see comment]," Am. J. Ind. Med., vol. 19, no. 1, pp. 87-107, 1991.

[121] A. Luttmann, M. Jäger, J. Sökeland, and W. Laurig, "Electromyographical study on surgeons in urology. II. Determination of muscular fatigue.," Ergonomics, vol. 39, no. 2, pp. 298-313, 1996.

[122] I. Jonkers, G. Nuyens, J. Seghers, M. Nuttin, and A. Spaepen, "Muscular effort in multiple sclerosis patients during powered wheelchair manoeuvres," Clin. Biomech., vol. 19, no. 9, pp. 929938, 2004.

[123] M. Cifrek, V. Medved, S. Tonković, and S. Ostojić, "Surface EMG based muscle fatigue evaluation in biomechanics," Clin. Biomech., vol. 24, no. 4, pp. 327-340, 2009.

[124] M. I. Sabri, M. F. Miskon, and M. R. Yaacob, "Robust Features Of Surface Electromyography Signal," IOP Conf. Ser. Mater. Sci. Eng., vol. 53, p. 012019, 2013.

[125] D. P. Zwambag and S. H. M. Brown, "The Effect of Contralateral Submaximal Contraction on the Development of Biceps Brachii Muscle Fatigue," Hum. Factors, vol. 57, no. 3, pp. 461-470, 2015.

[126] M. R. Al-Mulla, F. Sepulveda, and M. Colley, "A review of noninvasive techniques to detect and predict localised muscle fatigue," Sensors, vol. 11, no. 4, pp. 3545-3594, 2011.

[127] B. Y. G. Gordon and a H. S. Holbournt, "IN A CONTRACTING MUSCLE their ears during strong contraction of the jaw muscles when the external," Most, no. 948, pp. 456-464, 1947. 
[128] C. Orizio, M. Gobbo, B. Diemont, F. Esposito, and A. Veicsteinas, "The surface mechanomyogram as a tool to describe the influence of fatigue on biceps brachii motor unit activation strategy. Historical basis and novel evidence," Eur. J. Appl. Physiol., vol. 90, no. 3-4, pp. 326-336, 2003.

[129] D. T. Barry, "Vibrations and sounds from evoked muscle twitches.," Electromyogr. Clin. Neurophysiol., vol. 32, no. 1-2, pp. 35-40, 1992.

[130] R. D. Hill and W. J. Armstrong, "Clinical applications of mechanomyography: technical brief," Free Libr., 2009.

[131] C. Orizio, "Muscle sound: bases for the introduction of a mechanomyographic signal in muscle studies.," Crit. Rev. Biomed. Eng., vol. 21, no. 3, pp. 201-243, 1993.

[132] E. Krueger, E. M. Scheeren, G. N. Nogueira-Neto, V. L. D. S. N. Button, and P. Nohama, "Advances and perspectives of mechanomyography," Rev. Bras. Eng. Biomed., vol. 30, no. 4, pp. 384-401, 2014.

[133] D. T. Barry, S. R. Geiringer, and R. D. Ball, "Acoustic myography: A noninvasive monitor of motor unit fatigue," Muscle Nerve, vol. 8, no. 3, pp. 189-194, 1985.

[134] T. W. Beck et al., "Mechanomyographic and electromyographic amplitude and frequency responses during fatiguing isokinetic muscle actions of the biceps brachii.," Electromyogr. Clin. Neurophysiol., vol. 44, no. 7, pp. 431-41.

[135] S. R. PERRY-RANA, T. J. HOUSH, G. O. JOHNSON, A. J. BULL, and J. T. CRAMER, "MMG and EMG Responses during 25 Maximal, Eccentric, Isokinetic Muscle Actions," Med. Sci. Sport. Exerc., vol. 35, no. 12, pp. 2048-2054, Dec. 2003.

[136] S. R. Perry-Rana, T. J. Housh, G. O. Johnson, A. J. Bull, J. M. Berning, and J. T. Cramer, "MMG and EMG responses during fatiguing isokinetic muscle contractions at different velocities," Muscle and Nerve, vol. 26, no. 3, pp. 367-373, 2002.

[137] D. M. Mancini, L. Bolinger, H. Li, K. Kendrick, B. Chance, and J. R. Wilson, "Validation of near-infrared spectroscopy in humans," J. Appl. Physiol., vol. 77, no. 6, 1994. 
[138] J. Taelman, J. Vanderhaegen, M. Robijns, G. Naulaers, A. Spaepen, and S. Van Huffel, "Estimation of Muscle Fatigue Using Surface Electromyography and Near-Infrared Spectroscopy," Springer, Boston, MA, 2011, pp. 353-359.

[139] J. Shi, Q. Chang, and Y.-P. Zheng, "Feasibility of controlling prosthetic hand using sonomyography signal in real time: Preliminary study," J. Rehabil. Res. Dev., vol. 47, no. 2, p. 87, 2010.

[140] J. Shi, Y. P. Zheng, X. Chen, and Q. H. Huang, "Assessment of muscle fatigue using sonomyography: Muscle thickness change detected from ultrasound images," Med. Eng. Phys., vol. 29, no. 4, pp. 472-479, 2007.

[141] A. E. Trejo, K. N. Done', A. A. DiMartino, D. Oleynikov, and M. S. Hallbeck, "Articulating vs. conventional laparoscopic grasping tools-surgeons' opinions," Int. J. Ind. Ergon., no. 36, pp. 25-35, 2006.

[142] M. J. Van Det, W. J. H. J. Meijerink, C. Hoff, E. R. Totté, and J. P. E. N. Pierie, "Optimal ergonomics for laparoscopic surgery in minimally invasive surgery suites: A review and guidelines," Surg. Endosc., vol. 23, no. 6, pp. 1279-1285, 2009.

[143] R. Berguer, J. Chen, and W. D. Smith, "A comparison of the physical effort required for laparoscopic and open surgical techniques.," Arch. Surg., vol. 138, no. 9, pp. 967-970, 2003.

[144] U. Matern, G. Kuttler, C. Giebmeyer, P. Waller, and M. Faist, "Ergonomic aspects of five different types of laparoscopic instrument handles under dynamic conditions with respect to specific laparoscopic tasks: an electromyographic-based study.," Surg. Endosc., vol. 18, no. 8, pp. 1231-1241, 2004.

[145] R. Berguer, S. Gerber, G. Kilpatrick, M. Remler, and D. Beckley, “A comparison of forearm and thumb muscle electromyographic responses to the use of laparoscopic instruments with either a finger grasp or a palm grasp.," Ergonomics, vol. 42, no. 12, pp. 1634-1645, 1999.

[146] H. J. Hermens and B. Freriks, "SENIAM 5. Recommendations for Sensors and Sensor Placement Procedures," Roessingh Research and Development. Enschede, the Netherlands, 1997.

[147] C. J. De Luca, "Surface ELectromyography : Detection and Recording," DelSys Inc., vol. 10, no. 2, pp. 1-10, 2002. 
[148] T. Y. Fukuda et al., "Root mean square value of the electromyographic signal in the isometric torque of the quadriceps, hamstrings and brachial biceps muscles in female subjects," J. Appl. Res., vol. 10, no. 1, pp. 32-39, 2010.

[149] van Veelen, Jakimowicz, and Kazemier, "Improved physical ergonomics of laparoscopic surgery.," Minim. invasive Ther. allied Technol., vol. 13, no. 3, pp. 161-166, 2004.

[150] K. D. Tung, R. M. Shorti, E. C. Downey, D. S. Bloswick, and A. S. Merryweather, "The effect of ergonomic laparoscopic tool handle design on performance and efficiency," Surg. Endosc., vol. 29, no. 9, pp. 2500-2505, 2015.

[151] B. Steinhilber et al., "Ergonomic Benefits From a Laparoscopic Instrument With Rotatable Handle Piece Depend on the Area of the Operating Field and Working Height," Hum. Factors, vol. 59, no. 7, pp. 1048-1065, 2017.

[152] T. Wei, B. Lee, Y. Qiao, A. Kitsikidis, K. Dimitropoulos, and N. Grammalidis, "Experimental study of skeleton tracking abilities from microsoft kinect non-frontal views," 3DTV-Conference, vol. 2015-July, no. 1, pp. 1-4, 2015.

[153] A. Menache, "Motion Capture Primer," Underst. Motion Capture Comput. Animat., pp. 1-46, 2011.

[154] H. Mousavi Hondori and M. Khademi, "A Review on Technical and Clinical Impact of Microsoft Kinect on Physical Therapy and Rehabilitation," J. Med. Eng., vol. 2014, pp. 1-16, 2014.

[155] J. Richards, "The measurement of human motion: A comparison of commercially avaiable systems," Hum. Mov. Sci., vol. 18, pp. 589-602, 1999.

[156] A. Ali, K. Sundaraj, B. Ahmad, N. Ahamed, and A. Islam, "Gait disorder rehabilitation using vision and non-vision based sensors: A systematic review," Bosn. J. Basic Med. Sci., vol. 12, no. 3, pp. 193-202, 2012.

[157] W. Potthast and G. Brueggemann, "Comparison of Sprinting Mechanics of the Double Transtibial Amputee Oscar Pistorius With Able Bodied Athletes," XXVIII Int. Symp. Biomech. Sport., no. July, pp. 121-123, 2010. 
[158] G. Shan and P. Westerhoff, "Full-body kinematic characteristics of the maximal instep Soccer kick by male soccer players and parameters related to kick quality," Sport. Biomech., vol. 4, no. 1, pp. 59-72, 2005.

[159] T. J. Neves, W. A. Johnson, J. William Myrer, and M. K. Seeley, "Comparison of the traditional, swing, and chicken wing volleyball blocking techniques in NCAA division I female athletes," J. Sport. Sci. Med., vol. 10, no. 3, pp. 452-457, 2011.

[160] Y. V. Kolwadkar, S. I. Brown, R. J. Abboud, and W. Wang, "Comparison of two actuation systems for laparoscopic surgical manipulators using motion analysis," Surg. Endosc. Other Interv. Tech., vol. 25, no. 3, pp. 964-974, 2011.

[161] R. Hernandez, F. Travascio, A. Onar-Thomas, and S. S. Asfour, "Effect of Visual Display Location on Human Performance in Simulated Laparoscopic Tasks," J. Ergon., vol. 04, no. 03, 2014.

[162] M. A. Laribi, M. Arsicault, T. Riviere, and S. Zeghloul, "Toward new minimally invasive surgical robotic system," 2012 IEEE Int. Conf. Ind. Technol. ICIT 2012, Proc., no. May 2014, pp. 504-509, 2012.

[163] L. Yang, L. Zhang, H. Dong, A. Alelaiwi, and A. Saddik, "Evaluating and improving the depth accuracy of Kinect for Windows v2," IEEE Sens. J., vol. 15, no. 8, pp. 4275-4285, 2015.

[164] J. Salvi, S. Fernandez, T. Pribanic, and X. Llado, "A state of the art in structured light patterns for surface profilometry," Pattern Recognit., vol. 43, no. 8, pp. 2666-2680, 2010.

[165] T. Dutta, "Evaluation of the Kinect sensor for 3-D kinematic measurement in the workplace," Appl. Ergon., vol. 43, no. 4, pp. 645-649, 2012.

[166] S. Phommahavong, D. Haas, J. Yu, S. Krüger-Ziolek, K. Möller, and J. Kretschmer, "Evaluating the microsoft kinect skeleton joint tracking as a tool for home-based physiotherapy," Curr. Dir. Biomed. Eng., vol. 1, no. 1, pp. 184-187, 2015.

[167] L. F. Yeung, K. C. Cheng, C. H. Fong, W. C. C. Lee, and K.-Y. Tong, "Evaluation of the Microsoft Kinect as a clinical assessment tool of body sway.," Gait Posture, vol. 40, no. 4, pp. 532-8, 2014. 
[168] S. Obdrzalek et al., "Accuracy and robustness of Kinect pose estimation in the context of coaching of elderly population," Proc. Annu. Int. Conf. IEEE Eng. Med. Biol. Soc. EMBS, pp. 1188-1193, 2012.

[169] A. Fernández-Baena, A. Susín, and X. Lligadas, "Biomechanical validation of upper-body and lower-body joint movements of kinect motion capture data for rehabilitation treatments," Proc. 2012 4th Int. Conf. Intell. Netw. Collab. Syst. INCoS 2012, pp. 656-661, 2012.

[170] J. A. Diego-Mas and J. Alcaide-Marzal, "Using Kinect sensor in observational methods for assessing postures at work," Appl. Ergon., vol. 45, no. 4, pp. 976-985, 2014.

[171] A. Maimone and H. Fuchs, "Encumbrance-free telepresence system with real-time 3D capture and display using commodity depth cameras," 2011 10th IEEE Int. Symp. Mix. Augment. Reality, ISMAR 2011, pp. 137-146, 2011.

[172] D. Brown, "Video Modeling : Combining Dynamic Model Simulations with Traditional Video Analysis," in American Association of Physics Teachers 2008, 2008.

[173] X. Jiang, B. Zheng, and M. S. Atkins, "Video Processing to Locate the Tooltip Position in Surgical Eye-Hand Coordination Tasks.," Surg. Innov., pp. 1-9, 2014.

[174] R. Berguer, S. Gerber, G. Kilpatrick, and D. Beckley, "An ergonomic comparison of in-line vs pistol-grip handle configuration in a laparoscopic grasper.," Surg. Endosc., vol. 12, no. 6, pp. 805-808, 1998.

[175] K. Chandrasekaran and A. Thondiyath, "Design of a Two Degreeof-Freedom Compliant Tool Tip for a Handheld Powered Surgical Tool," J. Med. Device., vol. 11, no. 1, p. 014502, 2016.

[176] J. G. Betts et al., Anatomy \& Physiology. 2013.

[177] S. Hignett and L. McAtamney, "Rapid entire body assessment (REBA).," Appl. Ergon., vol. 31, no. 2, pp. 201-205, 2000.

[178] L. McAtamney and E. Nigel Corlett, "RULA: a survey method for the investigation of work-related upper limb disorders," Appl. Ergon., vol. 24, no. 2, pp. 91-99, 1993. 
[179] C. Y. Kim, J. S. Hong, and K. J. Chun, "Validation of feasibility of two depth sensor-based Microsoft Kinect cameras for human abduction-adduction motion analysis," Int. J. Precis. Eng. Manuf., vol. 17, no. 9, pp. 1209-1214, 2016.

[180] H. E. McLoone, M. Jacobson, C. Hegg, and P. W. Johnson, "Usercentered design," Work, vol. 37, no. 4, pp. 445-456, 2010.

[181] F. E. Ritter, G. D. Baxter, and E. F. Churchill, "Foundations for Designing User-Centered Systems," 2014.

[182] J. D. Gould and C. Lewis, "Designing for usability: key principles and what designers think," Commun. ACM, vol. 28, no. 3, pp. 300-311, 1985.

[183] J. M. C. Bastien, "Usability testing: a review of some methodological and technical aspects of the method," Int. J. Med. Inform., vol. 79, no. 4, pp. e18-e23, 2010.

[184] G. Ginsburg, "Human factors engineering: A tool for medical device evaluation in hospital procurement decision-making," J. Biomed. Inform., vol. 38, no. 3, pp. 213-219, 2005.

[185] A. W. Kushniruk, M. M. Triola, E. M. Borycki, B. Stein, and J. L. Kannry, "Technology induced error and usability: The relationship between usability problems and prescription errors when using a handheld application," Int. J. Med. Inform., vol. 74, no. 7-8, pp. 519-526, 2005.

[186] M. C. Beuscart-Zéphir, S. Pelayo, F. Anceaux, J. J. Meaux, M. Degroisse, and P. Degoulet, "Impact of CPOE on doctor-nurse cooperation for the medication ordering and administration process," Int. J. Med. Inform., vol. 74, no. 7-8, pp. 629-641, 2005.

[187] P. Elkin, S. Pelayo, and R. Beuscart, "The Human Factors Engineering Approach to Biomedical Informatics Projects : State of the Art, Results , Benefits and Challenges," pp. 109-127, 2007.

[188] D. A. Norman, "Human-centered design considered harmful," Interactions, vol. 12, no. 4, p. 14, 2005.

[189] U.S. Department of Health \& Human Services, "usability.gov," User-Centered Design Basics. [Online]. Available: https://www. usability.gov/what-and-why/user-centered-design.html. 
[190] D. Rueda, R. Hoto, and A. Conejero, "Study of the influence of prototype aesthetic fidelity (a realism factor) in usability tests," Lect. Notes Comput. Sci. (including Subser. Lect. Notes Artif. Intell. Lect. Notes Bioinformatics), vol. 7946 LNCS, pp. 122-136, 2013.

[191] J. Sauer, K. Seibel, and B. Rüttinger, "The influence of user expertise and prototype fidelity in usability tests," Appl. Ergon., vol. 41, no. 1, pp. 130-140, 2010.

[192] R. Virzi, J. L. Sokolov, and D. Karis, "Usability problem identification using both low- and high-fidelity prototypes," Proc. SIGCHI Conf. Hum. factors Comput. Syst. common Gr., pp. 236-243, 1996.

[193] M. McCurdy, C. Connors, G. Pyrzak, B. Kanefsky, and A. Vera, "Breaking the Fidelity Barrier - An Examination of our Current Characterization of Prototypes and an Example of a Mixed-Fidelity Success," Proc. Int. Conf. Hum. Factors Comput. Syst., pp. 12331242, 2006. 

Acknowledgments 
Me gustaría expresar mi enorme gratitud a mis dos directores, Dr. Andrés Conejero y Dr. José Luis Martínez de Juan por darme la oportunidad de trabajar con ellos y por su ayuda en cada paso de este largo proceso. Agradezco que me ayudaran siempre que lo necesité y fueran pacientes, al final lo logramos! También me gustaría agradecer a Paco Dolz y todo el equipo de Simulación Clinica del Hospital La Fe, porque sin ellos no habriamos nunca entendido del todo el problema que tratábamos.

Agradezco profundamente a Miguel y Rene, grandes compañeros de fatigas en el IDF y mejores amigos. Sin las risas, las quejas, y sin darnos caña mutuamente habría dejado esto nada más empezar. Estos últimos años sin teneros cerca ha costado mucho seguir.

También agradezco el apoyo de mis grandes amigos tanto de Valencia (Juan, Carlos, Iñaki, Cesar,...) como de Alhama (Mati, Javi, Sito, Rober, Marina,...), los cuales he tenido el placer de tener en mi vida durante estos años y me han dado ánimos y me han permitido desconectar siempre que han tenido oportunidad.

Pero sobre todo quiero agradecer este trabajo a mi familia. Gracias gordos por no rendiros, por ser pacientes, por apoyarme y por ofrecerme solo facilidades hasta conseguirlo. Estoy seguro que tener que mantener hijos hasta los 30 no era el plan, os lo agradezco. Sin vosotros seguro que nunca habría llegado aquí. Y a ti monillo, te quiero mucho, me gusto tenerte como compañero de doctorado, pero me gusta más verte feliz ahora que ya no tienes que sufrirlo. Sinceramente, esto es para vosotros, porque a mí los títulos no me interesan, pero veros orgullosos vale todo el esfuerzo del mundo.

Finalmente, a mi compañera de aventuras, no tengo palabras para agradecer lo que has sido para mí estos años. Siempre digo que conocerte ha sido lo mejor de hacer un doctorado y sin duda es lo mejor que me llevo. Tú apoyo y tu cariño han sido vitales para mí, especialmente este último año, ha sido muy duro para mí, me he hundido más hondo y más veces que nunca en mi vida, pero tú siempre estabas ahí, como una lucecita que iluminaba el pozo indicándome el camino que tenía que seguir, mil gracias por todo. No puedo estar más orgulloso de la compañera que tengo ni más envidioso de la investigadora con la que me comparo. Gracias por estar a mi lado. 



\section{THE EFFECT OF "POSTURAL FREEDOM" IN LAPAROSCOPIC SURGERY}

Horacio M. Pace Bedetti

Valencia, 2019

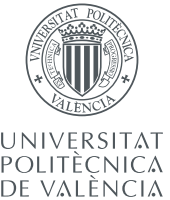

